\title{
Electron Neutrino and Antineutrino Appearance in the MINOS Detector
}

\author{
A DISSERTATION \\ SUBMITTED TO THE FACULTY OF THE GRADUATE SCHOOL \\ OF THE UNIVERSITY OF MINNESOTA \\ BY
}

Adam Paul Schreckenberger

IN PARTIAL FULFILLMENT OF THE REQUIREMENTS FOR THE DEGREE OF DOCTOR OF PHILOSOPHY

Professor Marvin Marshak, Faculty Advisor Assistant Professor Gregory Pawloski, Faculty Co-Adviser

April 2013 
This thesis is distributed under the terms of the Creative Commons Attribution License, which permits the use, distribution and reproduction of this material so long as the author and source are credited.

(C) Adam Paul Schreckenberger 2013 


\section{Acknowledgements}

I would like to thank my advisors, Marvin Marshak \& Greg Pawloski, my professors at the University of Minnesota, the members of the department's neutrino group, the members of the MINOS electron neutrino appearance group, and my friends in the CDP - for the betterment of all mankind. All of these individuals greatly contributed to my graduate school experience. 


\section{Dedication}

This dissertation is dedicated to my parents, Ann \& Paul Schreckenberger; my great friend, Derrick Brace; my above \& beyond supporter, Julie Harrison; and Sjuju, the celestial whale overseer.

In memory of SPC Lee S. Atkins.

1988-2012 


\begin{abstract}
The Main Injector Neutrino Oscillation Search (MINOS) is a long-baseline neutrino experiment that utilizes a particle beam and two steel-scintillator calorimeters designed to determine the parameters associated with muon neutrino disappearance. Analysis methods developed by the MINOS $v_{\mathrm{e}}$ group have facilitated the placement of limits upon the mixing angle associated with $v_{\mu} \rightarrow v_{\mathrm{e}}$ oscillations. Since the polarity of the focusing horns can be switched, we can perform a similar analysis with an antineutrino-enriched beam to select electron antineutrino appearance candidates. Using 3.34e20 POT (protons on target) in the antineutrino mode, we exclude $\theta_{13}=0$ at the $80 \%$ C.L. A joint fit of the 3.34e20 POT antineutrino and 10.6e20 POT neutrino samples excluded $\theta_{13}=0$ at the 96\% C.L. In addition, the combined data were used to produce exclusions regarding the CP-violating phase.
\end{abstract}




\section{Table of Contents}

T. List of Tables vii

F. List of Figures viii

1. Introduction 1

2. Neutrino Physics 3

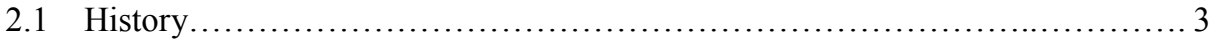

2.2 Beyond the Standard Model \& Motivations in Neutrino Physics................... 6

2.3 Oscillation Theory.......................................................... 7

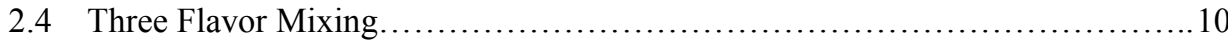

2.5 Inclusion of Matter Effects................................................... 11

2.6 Constructing the Full Formalism................................................. 14

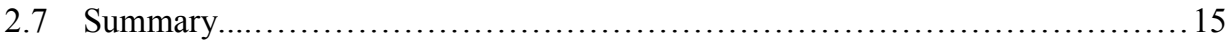

3. The NuMI Beam 16

4. The MINOS Experiment 23

4.1 General Detector Characteristics.............................................. 23

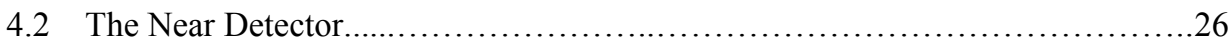

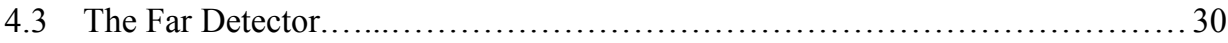

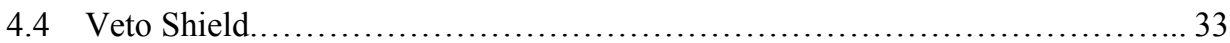

4.5 The Calibration Detector................................................... 34

4.6 Event Types and Reconstruction......................................... 34

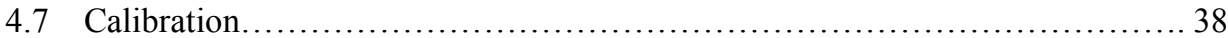

4.8 Monte Carlo..................................................................... 42 
5.1 Historical Summary........................................................ 46

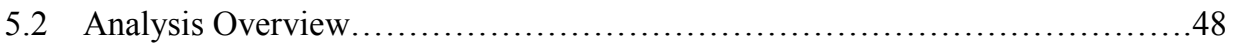

5.3 Analysis Bin Selection Study ............................................ 56

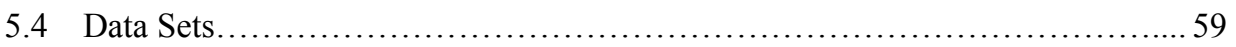

6. Library Event Matching (LEM) Particle ID Algorithm 61

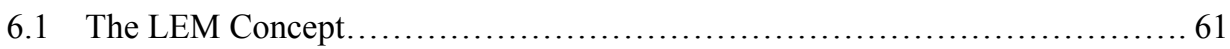

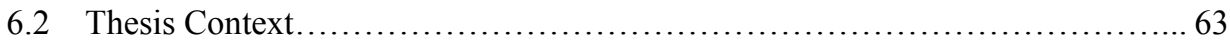

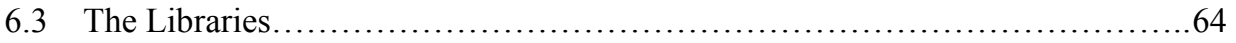

6.4 Match Preparation.................................................................65

6.5 Candidate Preselection................................................... 72

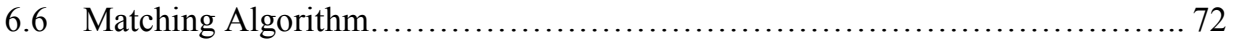

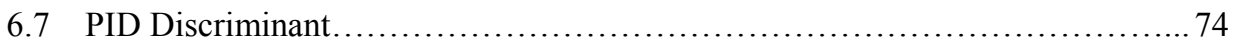

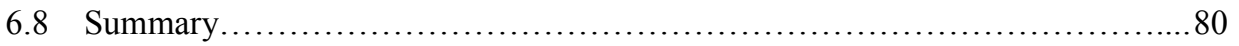

7. Systematic Errors $\quad 82$

7.1 Simulating the Systematic Errors.............................................. 82

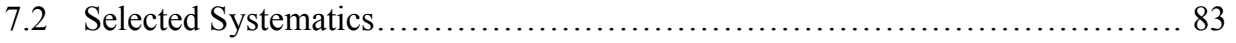

7.3 Total Uncertainties..................................................... 91

8. Far Detector Prediction 92

$8.1 \mathrm{NC}, v_{\mu} \mathrm{CC}$, and Beam $v_{e}$ Backgrounds...................................... 92

$8.2 v_{\tau} \mathrm{CC}$ and Signal $v_{e} \mathrm{CC}$ Predictions......................................... 93

8.3 Selection Efficiency Correction................................................ 94

8.4 Near Detector Results \& Making the Far Detector Prediction.................. 96 
9. Fitting Procedures for the Far Detector Data 103

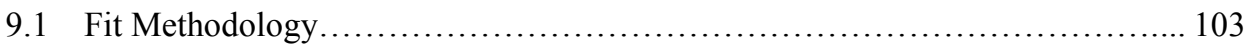

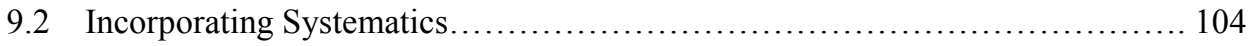

9.3 Feldman-Cousins Prescription............................................. 105

$\begin{array}{ll}\text { 10. Far Detector Data and Results } & 107\end{array}$

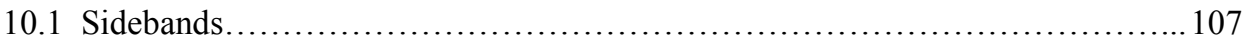

10.2 Unblinded Data................................................................... 115

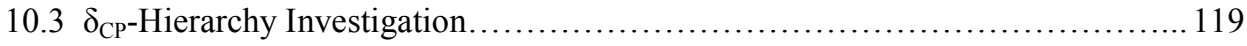

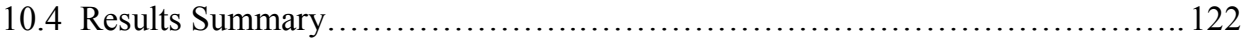

11. Welcome to the World of Tomorrow 124

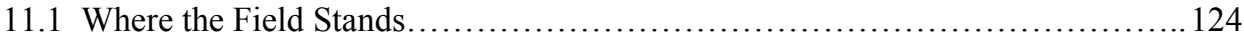

11.2 Where the Field is Going ................................................. 128

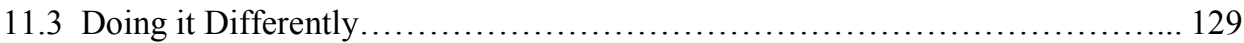

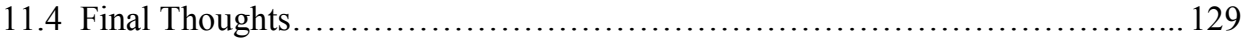

$\begin{array}{ll}\text { R. References } & 131\end{array}$ 


\section{List of Tables}

Table 2.1 Current best fit values of the neutrino oscillation parameters.................11

Table 5.1 Fiducial volumes of the MINOS detectors............................... 50

Table 5.2 POT exposures for the MINOS run periods ................................60

Table 7.1 Changes in sensitivity from energy scale systematics.......................84

Table 7.2 Fractional uncertainties on the standard backgrounds..................... 84

Table 7.3 Absolute energy scale fractional uncertainties in the prediction.............85

Table 7.4 EMvsHad fractional uncertainties in the prediction.........................86

Table 7.5 Normalization error fractional uncertainties in the prediction..............86

Table 7.6 Neutrino cross section fractional uncertainties in the prediction.......... 87

Table 7.7 Hadronic modeling fractional uncertainties in the prediction..............89

Table $7.8 v_{\mu}$ CC spectrum fractional uncertainties in the prediction..................89

Table 7.9 Antineutrino vs. neutrino flux uncertainty investigation .................... 90

Table 7.10 Total systematic uncertainties........................................... 91

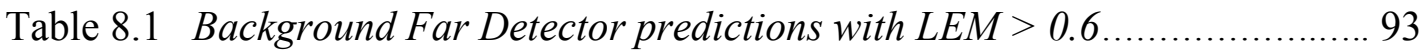

Table 8.2 Far Detector predictions with LEM > 0.6 for oscillation events............94

Table 8.3 Efficiency correction factors ............................................ 95 


\section{List of Figures}

Figure $2.1 \quad$ Flux of ${ }^{8} B$ solar neutrinos..................................................... 5

Figure 2.2 The mass hierarchy problem .........................................

Figure 2.3 Feynman diagrams of NC and CC neutrino interactions.................. 12

Figure $3.1 \quad$ NuMI protons delivered to the graphite target over time ................... 16

Figure 3.2 Technical drawing of the NuMI target.................................... 17

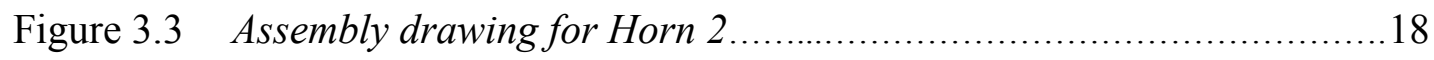

Figure 3.4 Sign-selected focusing of secondary mesons............................... 18

Figure 3.5 Simulated event rate of $v_{\mu}$ CC interactions in the Near Detector........ 20

Figure 3.6 Plan and elevation views of the NuMI beam facility.......................21

Figure 3.7 Energy spectra of neutrino events in the Near Detector....................22

Figure 4.1 Illustration depicting the MINOS steel and scintillator planes...........25

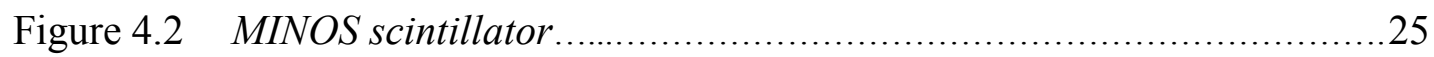

Figure 4.3 Diagram of the readout assembly........................................ 26

Figure 4.4 Configurations of Near Detector scintillator planes.......................28

Figure 4.5 Picture of the MINOS Near Detector ...................................29

Figure 4.6 Plane configurations of the Far Detector................................... 31

Figure 4.7 Picture of the MINOS Far Detector....................................... 32

Figure 4.8 Monte Carlo displays of $v_{\mu} C C, v_{e} C C$, and $N C$ events.................. 35

Figure 4.9 Flow diagram of the two calibration chains used by MINOS............. 38

Figure $5.12 \sin ^{2}\left(2 \theta_{13}\right) \sin ^{2} \theta_{23}$ contours from the 2011 appearance search........... 47

Figure 5.2 MINOS antineutrino disappearance analyses ............................ 48 
Figure 5.3 Far Detector MC study from the second appearance analysis.....

Figure 5.4 Far Detector MC study of track plane and track-like plane cuts........ 52

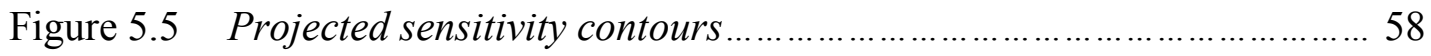

Figure 6.1 Flowchart of a LEM PID value assignment .................................62

Figure 6.2 Candidate event compared to two LEM library events.................... 63

Figure 6.3 Pre-corrected light-levels produced by simulated muon events.......... 67

Figure 6.4 Corrected light-levels of simulated muon events.........................68

Figure 6.5 Event compacting strip groupings ...................................... 70

Figure 6.6 Event displays through compacting process............................. 71

Figure 6.7 Distributions of $y_{50}, q_{50}, f_{50}$, and reco E from FHC training sample... 76

Figure 6.8 Distributions of $y_{50}, q_{50}, f_{50}$, and reco E from RHC training sample.... 77

Figure 6.9 Area Normalized PID value distributions of MC test sample.............80

Figure 8.1 Results of the Near Detector beam decomposition - Set I................97

Figure 8.2 Results of the Near Detector beam decomposition - Set II.............. 97

Figure 8.3 Results of the Near Detector beam decomposition - Set III...............98

Figure 8.4 Stacked histograms of the predicted joint Far Detector spectrum .......99

Figure 8.5 Stacked histograms of the predicted RHC Far Detector spectrum....... 100

Figure 8.6 Near Detector and predicted Far Detector LEM PID distributions.....101

Figure 10.1 LEM PID distribution in the FHC Anti-PID ................................ 108

Figure 10.2 LEM PID distribution in the RHC Anti-PID sideband..................... 109

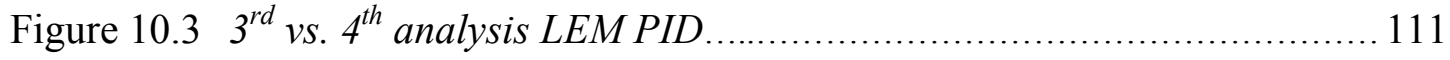

Figure 10.4 RHC and FHC MRCC Sideband PID distributions ....................... 114 
Figure 10.5 Far Detector LEM PID distributions for FHC and RHC modes.........116

Figure 10.6 MINOS RHC only Feldman-Cousins contours............................ 117

Figure 10.7 MINOS FHC+RHC Feldman-Cousins contours......................... 118

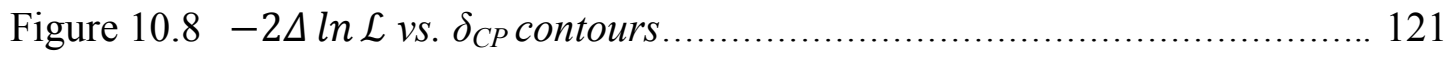

Figure 11.1 Overlaid 90\% C.L. regions from MINOS 2011 and T2K 2011_......... 126

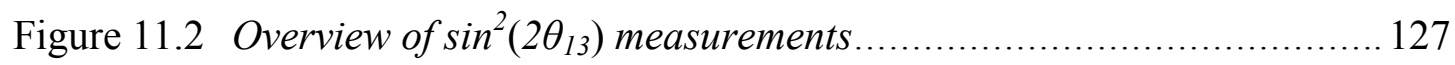




\section{Chapter 1}

\section{Introduction}

While much of the world's attention is focused on the results of energy frontier physics experiments, some of the most exciting scientific results have come from neutrino research and the development of the high intensity frontier. Neutrinos are one of the most abundant particles in our universe; they are the products of nuclear decay, they are produced in our sun, and some are even relics of the Big Bang. Until recently, however, the overwhelming presence of these particles did not match our level of understanding of the phenomena, physics and mathematics that govern their existences. Because neutrinos interact only through the weak force, they are harder to detect in comparison with other, more familiar particles.

Our understanding of the neutrino had relatively humble beginnings as a construct meant to explain the missing energy observed in beta-decay, but the impact of this little lepton could not be larger. The current version of the Standard Model, the framework through which we explain physical interactions and the particles that we see, did not predict a nonzero neutrino mass. However, the discovery and subsequent confirmations of neutrino oscillations demonstrated that neutrinos had just that. Decades ago, this was a subject of debate within the physics community, but today, it is taken as commonplace. Neutrinos do undergo flavor oscillation, and they do have nonzero mass.

This past year has proven to be an exciting time to be a neutrino physicist. Up until recently, the mixing angle $\theta_{13}$ was unknown and was thought to be small. This particular parameter is important to the community, for a nonzero $\theta_{13}$ facilitates the incorporation of 
$\mathrm{CP}$-violation in the lepton sector. Such a discovery would have a profound impact upon our understanding of the universe, for such a discovery offers a potential glimpse into the matter-antimatter asymmetry problem.

Reactor experiments (Daya Bay, RENO and Double Chooz) produced compelling evidence that $\theta_{13}$ is nonzero. ${ }^{(1)(2)(3)}$ The statistical significances of these discoveries provide additional assurance that new oscillation modes have been observed. Within months, $\theta_{13}$ went from being an unknown constant to one of the most precisely known parameters in neutrino physics. These results, along with the preceding interest in measuring $\theta_{13}$, have encouraged the development of the next generation of acceleratorbased oscillation experiments.

MINOS is capable of making a measurement of $\theta_{13}$ by searching for evidence of $v_{\mu} \rightarrow v_{e}$ oscillations between its two neutrino detectors. In addition, further constraints upon the relevant parameters are placed by searching for the corresponding antiparticle transition. This thesis aims to present the updated measurement of $\theta_{13}$ as well as the information gathered about the neutrino mass hierarchy and CP-violating phase.

As MINOS was not optimized for an electron neutrino appearance search, the experiment faces challenges in regards to these analyses. The similar topologies of signal events with dominating background contaminants and the resolution of the detector has necessitated the development of special selection techniques to identify $v_{e}$ and $\bar{v}_{e}$ candidates. These techniques will be described in this dissertation in addition to the methods used to identify our $\theta_{13}$ confidence intervals and exclusions in the $\delta_{\mathrm{CP}}$-hierarchy phase space. 


\section{Chapter 2}

\section{Neutrino Physics}

\subsection{History}

Neutrinos were first conceived by Pauli in 1930 as a means to explain the continuous energy spectrum of electrons in beta decays. As often seen in the field, the need to maintain conservation rules often facilitates the addition of some additional structure to preserve what are considered fundamental principles. The neutrino was the simple answer to Pauli's problem.

Given its neutral charge and the fact that it interacts only through the weak nuclear force, the neutrino was not directly observed until the Cowan \& Raines Project Poltergeist reactor experiment detected the electron antineutrino in $1956 .^{(4)}$ The muon neutrino was discovered in 1962 by Lederman, Schwartz and Steinberger; ${ }^{(5)}$ and the tau neutrino was detected in 2000 by the Direct Observation of the Nu-Tau (DONuT) collaboration. ${ }^{(6)}$ Pontecorvo conceptualized the possibility of neutrino oscillations in 1957 by generalizing the notions related to kaon mixing. As only one flavor of neutrino had been discovered at that time, Pontecorvo's hypothesis focused on mixing between $v$ and $\bar{v} .{ }^{(7)}$ In 1962 , with the knowledge that multiple flavors of neutrinos existed in nature, Maki, Nakagawa and Sakata proposed oscillations between $v_{e}$ and $v_{\mu} \cdot{ }^{(8)}$ Their framework was extended to include the tau-flavored neutrino, and today, the Pontecorvo-Maki-Nakagawa-Sagata (PMNS) neutrino mixing matrix honors their contributions to the field.

In 1967, the Homestake experiment - pioneered by Davis and Bahcall - uncovered the first indication that supported the neutrino mixing theory. They sought to measure the 
rate at which solar neutrinos were captured by chlorine nuclei. Bahcall predicted the frequency of this interaction to be $(2.0 \pm 1.2) \cdot 10^{-35} / \mathrm{s} /$ atom; however, the Homestake study established $0.3 \cdot 10^{-35} / \mathrm{s} /$ atom as its upper limit. ${ }^{(9)}$ This deficit was seen in subsequent experiments, but the source of the discrepancy remained unclear. Many pointed towards a misunderstanding in the solar model or scrutinized the neutrino experiments.

The deficit phenomenon, however, was not limited to solar neutrino observations. Atmospheric neutrino experiments sought to validate the approximately 2:1 ratio expected between muon and electron neutrinos that were produced through the $\pi \rightarrow \mu v_{\mu} \rightarrow e v_{e} v_{\mu} v_{\mu}$ decay chain in showers initiated by cosmic rays. The IrvineMichigan-Brookhaven (IMB) experiment, Monopole, Astrophysics and Cosmic Ray Observatory (MACRO), and the Kamiokande collaboration found significant deficits in the $v_{\mu}$ flux. In 1998, Super-Kamiokande explained the shortfall by fitting their results with a $v_{\mu} \leftrightarrow v_{\tau}$ neutrino oscillation framework. ${ }^{(10)}$

The debate in the solar neutrino sector ended in 2001 when the Sudbury Neutrino Observatory (SNO) experiment provided conclusive evidence that roughly two-thirds of the solar neutrino flux was related to non- $v_{e}$ flavors. This result supported the notion of neutrino oscillations and reconciled the total flux measurement with the standard solar model prediction. ${ }^{(11)}$ 


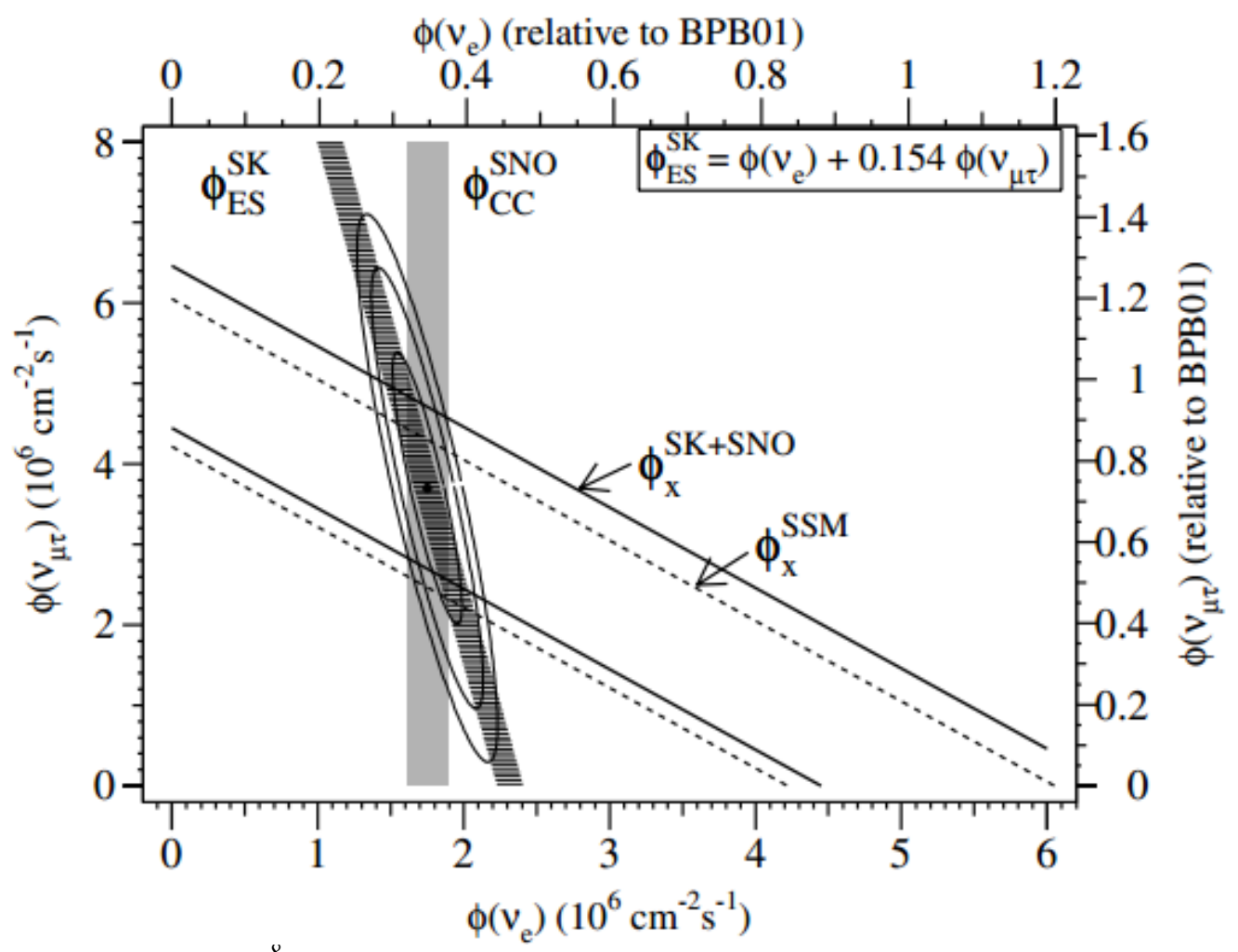

Figure 2.1: Flux of ${ }^{8} B$ solar neutrinos identified as either $\mu$ or $\tau$ flavored $v$ s. the flux of electron neutrinos. This result presented the first direct indication of a non-electron component of the solar neutrino flux, which supported the oscillation possibility explored by Super-Kamiokande. Image taken from (11).

Accelerator and reactor based oscillation studies emerged as major contributors in the last decade as physicists desired to measure the mixing parameters that described the neutrino oscillation phenomenon. MINOS used its muon neutrino beam to make the world-leading measurement of $\left|\Delta m_{23}^{2}\right|$, and the experiment has made a competitive measurement of $\theta_{23}$ as well. While reactor experiments produced the most statistically significant measurements of $\theta_{13}$ in 2012, the results of the MINOS analysis can yield important contributions to field. 


\subsection{Beyond the Standard Model \& Motivations in Neutrino Physics}

In the current Standard Model (SM) framework, neutrinos are regarded as massless, uncharged leptons that interact with matter only via the weak nuclear force. The observation of neutrino oscillations indicates that the SM picture does not provide a complete description of particles and their interactions. In this regard, neutrino physics demands additional research because a more accurate depiction of the universe must exist.

Neutrino oscillations introduce other worthwhile areas of investigation as well. It is known that $\mathrm{CP}$ violation occurs in the quark sector through the CKM mixing matrix; however, that is not enough to explain the lopsided matter-antimatter asymmetry observed today. ${ }^{(12)}$ Therefore, the possibility that $\mathrm{CP}$ violation occurs in the lepton sector via the neutrino oscillation matrix provides an exciting investigation to neutrino physicists, and this excitement is supported by various theories in particle physics. The leptogenesis handling of neutrinos, for example, offers a possible explanation of the matter-antimatter asymmetry if $\mathrm{CP}$ violation were detected in the oscillation phenomenon. ${ }^{(13)(14)}$

The absolute masses of neutrinos and the ordering of the mass-squared splittings are currently not known. The current upper limits placed upon the neutrino masses by decay experiments are as follows: $\mathrm{m}\left(v_{\mathrm{e}}\right)<2 \mathrm{eV}, \mathrm{m}\left(v_{\mu}\right)<0.19 \mathrm{MeV}$ and $\mathrm{m}\left(v_{\tau}\right)<18.2 \mathrm{MeV} .^{(15)}$ Cosmological observations put an upper limit on the $\Sigma m\left(v_{\alpha}\right)$ at $0.67 \mathrm{eV}$ at the $95 \%$ C.L. (16) Solar neutrino experiments established the ordering of the $1^{\text {st }}$ and $2^{\text {nd }}$ neutrino mass state; however, it is not yet known if the $3^{\text {rd }}$ mass state is the heaviest or lightest of the 
three. These two possibilities are respectively called the normal and inverted mass hierarchies.

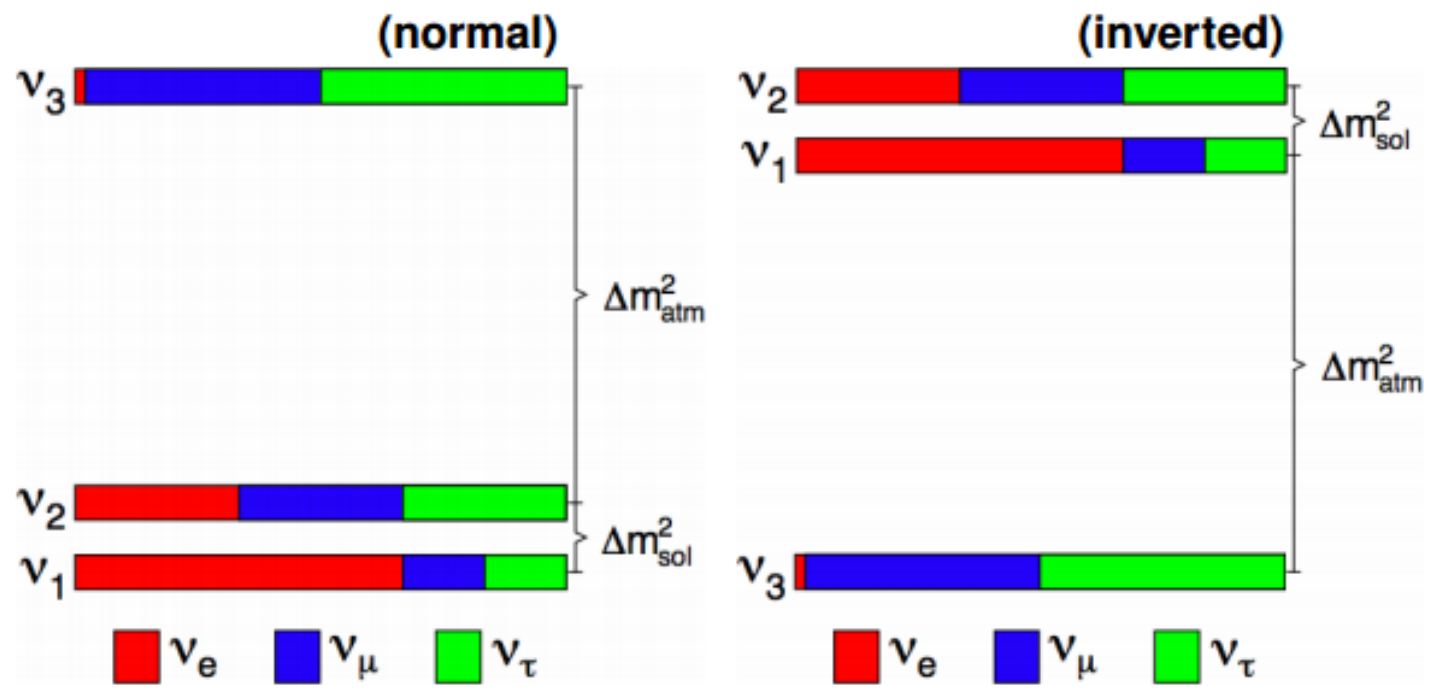

Figure 2.2: The mass hierarchy problem. This cartoon illustrates the three mass eigenstates, their respective flavor compositions, and the possible mass orderings. Image taken from (17).

Until recently, there were only hints that $\theta_{13}$ was nonzero. MINOS has access to making this particular measurement by searching for $\nu_{\mu} \leftrightarrow v_{\text {e }}$ oscillations. Due to the acceleratorbased nature of the MINOS experiment and its long neutrino beam baseline, MINOS also has the capability to comment on the neutrino mass hierarchy. CP violation studies are within the scope of MINOS's physics reach as well. Utilizing the best fit results from reactor antineutrino disappearance searches in conjunction with the experiment's tunable source, MINOS provides the first exclusions of $\delta_{\mathrm{CP}}$ with respect to each hierarchy possibility.

\subsection{Oscillation Theory}

Neutrino oscillations arise in nature because two fundamentally distinct sets of eigenstates characterize the neutrino. The mass eigenstates describe the particle's 
propagation, whereas the flavor eigenstates encapsulate the particle's interaction behavior. As neutrinos interact primarily through the weak force, the flavor eigenstates are commonly referred to in literature as the weak eigenstates. These two bases are related via the PMNS unitary mixing matrix as follows:

$$
\left|v_{\alpha}\right\rangle=\sum_{i} U_{\alpha i}^{*}\left|v_{i}\right\rangle,
$$

where $\mathrm{U}$ designates the PMNS matrix, $\alpha$ denotes flavor states (e, $\mu$ or $\tau$ ), and i represents mass states (1, 2 or 3). Expression (2.1) is a general relation independent of the number of states.

When neutrinos are created through weak interaction processes, they have a definite flavor state. Therefore, they can be described as a superposition of $n$ mass eigenstates, where $n$ represents the number of mass states that participate in the mixing theory. The propagation of the mass eigenstate is written in the following form:

$$
\left|v_{i}(t)\right\rangle=e^{-i\left(E_{i} t-\left|\vec{p}_{i}\right| \cdot L\right)}\left|v_{i}(0)\right\rangle,
$$

where $\mathrm{L}$ indicates the distance traveled from the neutrino source to the point of detection. The propagation of the flavor eigenstate is written as

$$
\begin{gathered}
\left|v_{\alpha}(t)\right\rangle=\sum_{i} U_{\alpha i}^{*} e^{-i\left(E_{i} t-\left|\vec{p}_{i}\right| \cdot L\right)}\left|v_{i}(0)\right\rangle= \\
\sum_{i} \sum_{\beta} U_{\alpha i}^{*} U_{\beta i} e^{-i\left(E_{i} t-\left|\vec{p}_{i}\right| \cdot L\right)}\left|v_{\beta}\right\rangle .
\end{gathered}
$$

Consequently, the probability of observing a $\beta$-flavored neutrino after an $\alpha$-flavored neutrino has propagated for time $t$ is represented by 2.4 .

$$
\left|\left\langle v_{\beta} \mid v_{\alpha}(t)\right\rangle\right|^{2}=\left|\sum_{i} U_{\alpha i}^{*} U_{\beta i} e^{-i\left(E_{i} t-\left|\vec{p}_{i}\right| \cdot L\right)}\right|^{2}
$$

The neutrino oscillation phenomenon can perhaps be best understood by deconstructing its quantum mechanical roots. Consider a neutrino created with a definite flavor state at $t$ 
$=0$. As the neutrino travels, the different components of the mass eigenstate superposition propagate differently, and the resulting interference effects extend back into the flavor basis. Another interpretation is that the propagating mass states represent a set of unmeasured transitional alternatives with respect to the flavor basis, which alters the probability of observing a neutrino with a given flavor at nonzero $t$.

As the neutrinos produced in the accelerator-physics environment are ultrarelativistic, a series of approximations may be applied to write 2.4 in the following form:

$$
\begin{gathered}
\left|\left\langle v_{\beta} \mid v_{\alpha}(t)\right\rangle\right|^{2}=\left|\sum_{i} U_{\alpha i}^{*} U_{\beta i} e^{-i m_{i}^{2} L / 2 E}\right|^{2} \\
=\sum_{i j} U_{\alpha j} U_{\beta j}^{*} U_{\alpha i}^{*} U_{\beta i} e^{-i \Delta m_{i j}^{2} L / 2 E} \\
=\delta_{\alpha \beta}+\sum_{i j} U_{\alpha j} U_{\beta j}^{*} U_{\alpha i}^{*} U_{\beta i}\left(e^{-i \Delta m_{i j}^{2} L / 2 E}-1\right) \\
P\left(v_{\alpha} \rightarrow v_{\beta}\right)=\delta_{\alpha \beta}-4 \sum_{i>j} \operatorname{Re}\left[U_{\alpha j} U_{\beta j}^{*} U_{\alpha i}^{*} U_{\beta i}\right] \sin ^{2}\left(\frac{\Delta m_{i j}^{2} L}{4 E}\right) \\
+2 \sum_{i>j} \operatorname{Im}\left[U_{\alpha j} U_{\beta j}^{*} U_{\alpha i}^{*} U_{\beta i}\right] \sin \left(\frac{\Delta m_{i j}^{2} L}{2 E}\right),
\end{gathered}
$$

where $\Delta m_{i j}^{2} \stackrel{\text { def }}{=} m_{i}^{2}-m_{j}^{2}$.

The formalism of Expression (2.5) is enlightening for a couple of reasons. First, the oscillatory behavior of the probability function is immediately apparent; and second, the observation of neutrino mixing indicates a nonzero neutrino mass state.

$$
\frac{\Delta m_{i j L}^{2} L}{4 E} \approx 1.267 \frac{\Delta m_{i j}^{2}}{e V^{2}} \frac{L}{k m} \frac{G e V}{E}
$$

It is conventional, after restoring the necessary powers of $\mathrm{c}$ and $\hbar$, to rewrite the phase in accordance with 2.6. 
It should be noted that, barring the discovery of any new physics, CPT-symmetry implies that $P\left(v_{\alpha} \rightarrow v_{\beta}\right)=P\left(\bar{v}_{\beta} \rightarrow \bar{v}_{\alpha}\right)$ This can be also expressed in the following manner: $P\left(v_{\alpha} \rightarrow v_{\beta}, U\right)=P\left(\bar{v}_{\alpha} \rightarrow \bar{v}_{\beta}, U^{*}\right)$, where, $\mathrm{U}^{*}$ designates $\mathrm{U}$ being replaced by its complex conjugate.

\subsection{Three Flavor Mixing}

In the case that three flavors participate in neutrino oscillations, the PMNS mixing matrix is characterized by four free parameters: the Euler angles $\theta_{12}, \theta_{23}$ and $\theta_{13}$ as well as the CP-violating phase, $\delta$ :

$$
\left(\begin{array}{ccc}
c_{12} c_{13} & s_{12} c_{13} & s_{13} e^{-i \delta} \\
-s_{12} c_{23}-c_{12} s_{23} s_{13} e^{i \delta} & c_{12} c_{23}-s_{12} s_{23} s_{13} e^{i \delta} & s_{23} c_{13} \\
s_{12} s_{23}-c_{12} c_{23} s_{13} e^{i \delta} & -c_{12} s_{23}-s_{12} c_{23} s_{13} e^{i \delta} & c_{23} c_{13}
\end{array}\right)
$$

where $\mathrm{s}_{\mathrm{ij}} \equiv \sin \left[\theta_{\mathrm{ij}}\right], \mathrm{c}_{\mathrm{ij}} \equiv \cos \left[\theta_{\mathrm{ij}}\right]$, and Majorana phases have been neglected. The three angles dictate the coupling between the flavor and mass states, and a nonzero or $\mathrm{n} \pi \delta$ indicates that neutrino oscillations violate $\mathrm{CP}$-symmetry. In addition, two Majorana phases $-\alpha_{1}$ and $\alpha_{2}-$ are nonzero if neutrinos are their own antiparticles.

Combining 2.7 with 2.5 , one can construct the probability expression for any neutrino flavor transition. The parameters of the PMNS mixing matrix are measured by comparing a no-oscillation prediction to the number of neutrino interactions observed in a detector volume. Table 2.1 lists the global best fits for each of those matrix elements. 


\begin{tabular}{c|c}
\hline Parameter & Best Fit Value \\
\hline $\sin ^{2} 2 \theta_{12}$ & $0.857 \pm 0.025$ \\
$\sin ^{2} 2 \theta_{23}$ & $>0.95$ \\
$\sin ^{2} 2 \theta_{13}$ & $0.098 \pm 0.013$ \\
$\Delta m_{21}^{2}$ & $+(7.50 \pm 0.20) \cdot 10^{-5} \mathrm{eV}^{2}$ \\
$\left|\Delta m_{32}^{2}\right|$ & $(2.32 \pm 0.12) \cdot 10^{-3} \mathrm{eV}^{2}$ \\
$\delta_{\mathrm{CP}}$ & - \\
\hline
\end{tabular}

Table 2.1: Current best fit values of the neutrino oscillation parameters. ${ }^{(15)}$

Up until this point, the mathematical treatment of neutrino oscillations has considered only propagation in vacuum. In order to generate a complete understanding of their observations, neutrino experiments have to consider the impact of the MikheyevSmirnov-Wolfenstein (MSW) - or matter - effect.

\subsection{Inclusion of Matter Effects}

As neutrinos pass through matter, there exists a chance that they will weakly interact with particles in their immediate surroundings. This is particularly apparent in solar neutrino observation experiments, for neutrinos created via the Sun's nuclear processes must travel through relatively dense regions of material. These interactions modify the energy of the neutrino by exposing the particle to a supplemental potential.

Neutral Current (NC) interactions, shown in Figure 2.3, introduce the following potential:

$$
V_{N C}= \pm \frac{G_{F} n_{n}}{\sqrt{2}}
$$

where $\mathrm{G}_{\mathrm{F}}$ is the Fermi constant and $\mathrm{n}_{\mathrm{n}}$ is the neutron number density. Since this interaction equally impacts all three neutrino flavors, it does not alter the oscillation probability and thus has no effect upon the MINOS experiment. 
Charged Current (CC) interactions, also shown in Figure 2.3, introduce a potential that depends on the electron density.

$$
V_{C C}= \pm \sqrt{2} G_{F} n_{e}
$$

This potential exclusively affects the propagation of the electron neutrino in a manner that is akin to light traveling through a medium. As I will show, this potential introduces an effective electron neutrino mass, which modifies the mass-squared splitting and hence impacts the oscillation phenomenon.

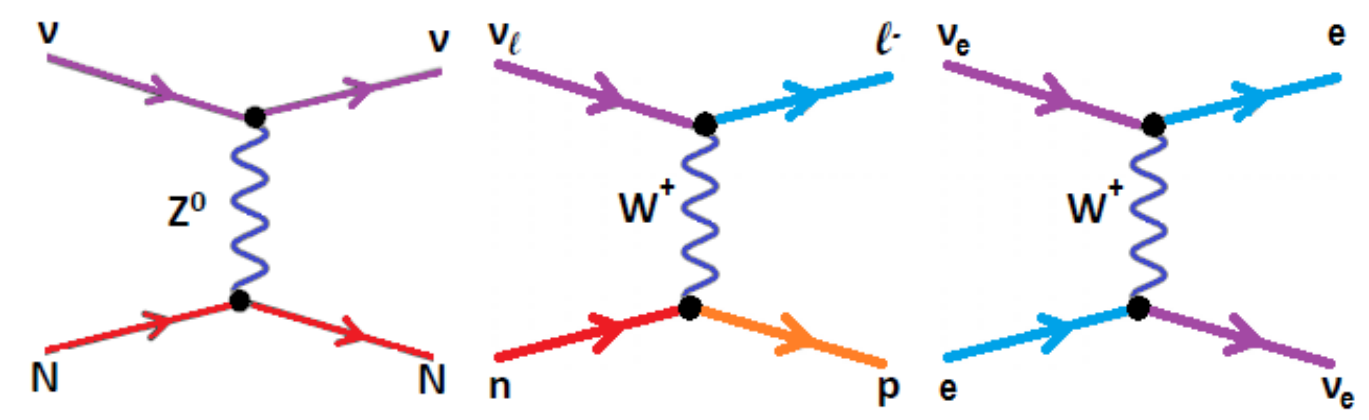

Figure 2.3: Feynman diagrams of neutral current $(N C)$ and charged current (CC) neutrino interactions. The far right picture illustrates the coherent forward scattering interaction which generates effective oscillation parameters via the MSW effect. In general, NC interactions involve the exchange of a neutral Z-boson, whereas CC interactions involve the $W$-boson instead.

To demonstrate the impact of the MSW effect upon neutrino oscillations, consider a twoneutrino model in which the charged current potential affects one of the neutrino flavors. The PMNS mixing matrix reduces to the form seen in Expression (2.3). $\mathrm{H}_{\mathrm{o}}$ is constructed by removing all terms proportional to the identity, for those terms do not affect neutrino oscillations.

$$
U=\left(\begin{array}{cc}
\cos \theta & \sin \theta \\
-\sin \theta & \cos \theta
\end{array}\right), H_{o}=\left(\begin{array}{cc}
m_{1}^{2} / 2 E & 0 \\
0 & m_{2}^{2} / 2 E
\end{array}\right)
$$


$\mathrm{H}_{\mathrm{o}}$ can be simplified further using the same argument. The following form is often used to condense the mathematics.

$$
H_{o}=\left(\begin{array}{cc}
0 & 0 \\
0 & \Delta m^{2} / 2 E
\end{array}\right)
$$

The Hamiltonian operator for a neutrino in vacuum can now be written as $\mathrm{H}=\mathrm{UH}_{\mathrm{o}} \mathrm{U}^{\dagger}$. Introducing the $\mathrm{CC}$ potential modifies the Hamiltonian in the following manner.

$$
H^{\prime}=H+V=\left(\begin{array}{cc}
\cos \theta & \sin \theta \\
-\sin \theta & \cos \theta
\end{array}\right)\left(\begin{array}{ll}
0 & 0 \\
0 & \Delta
\end{array}\right)\left(\begin{array}{cc}
\cos \theta & -\sin \theta \\
\sin \theta & \cos \theta
\end{array}\right)+\left(\begin{array}{ll}
V & 0 \\
0 & 0
\end{array}\right)
$$

where $\Delta \equiv \Delta \mathrm{m}^{2} / 2 \mathrm{E}$. In the expression below, I introduce the variable $\mathrm{C}$, which is defined as $\mathrm{V} / \Delta$.

$$
H^{\prime}=\Delta\left(\begin{array}{cc}
\sin ^{2} \theta+C & \sin \theta \cos \theta \\
\sin \theta \cos \theta & \cos ^{2} \theta
\end{array}\right)
$$

This new Hamiltonian can also be written and diagonalized in terms of effective mixing parameters. Once again, terms proportional to the identity are removed for simplicity.

$$
H^{\prime}=U\left(\theta_{\text {eff }}\right)\left(\begin{array}{cc}
0 & 0 \\
0 & \Delta\left(\lambda_{2}-\lambda_{1}\right)
\end{array}\right) U^{\dagger}\left(\theta_{\text {eff }}\right)
$$

Comparing 2.14 with 2.11 , one sees that the mass-squared splitting is modified by the $\lambda_{2}$ $-\lambda_{1}$ term. Therefore, solving for the eigenvalues of $\mathrm{H}^{\prime}$ yields the effective oscillation parameters for neutrinos propagating through matter.

$$
\begin{gathered}
\Delta m_{\text {eff }}^{2}=\Delta m^{2} \sqrt{\sin ^{2} 2 \theta+(\cos 2 \theta-C)^{2}} \\
\sin ^{2} 2 \theta_{\text {eff }}=\sin ^{2} 2 \theta / \sqrt{\sin ^{2} 2 \theta+(\cos 2 \theta-C)^{2}}
\end{gathered}
$$




\subsection{Constructing the Full Formalism}

With the mathematical framework of neutrino oscillations well in tow, the probability expressions pertaining to this MINOS analysis can be derived. Assuming a constant matter density and the normal mass hierarchy condition, the probability of a muon to electron neutrino transition is written as follows:

$$
\begin{aligned}
& P\left(v_{\mu} \rightarrow v_{e}, N H\right) \approx \sin ^{2} \theta_{23} \frac{\sin ^{2} 2 \theta_{13}}{(A-1)^{2}} \sin ^{2}((A-1) \Delta) \\
& +\alpha \frac{\sin \delta_{C P} \cos \theta_{13} \sin 2 \theta_{12} \sin 2 \theta_{13} \sin 2 \theta_{23}}{A(1-A)} \sin \Delta \sin A \Delta \sin ((1-A) \Delta) \\
& +\alpha \frac{\cos \delta_{C P} \cos \theta_{13} \sin 2 \theta_{12} \sin 2 \theta_{13} \sin 2 \theta_{23}}{A(1-A)} \cos \Delta \sin A \Delta \sin ((1-A) \Delta) \\
& +\alpha^{2} \frac{\cos ^{2} \theta_{23} \sin ^{2} 2 \theta_{12}}{A^{2}} \sin ^{2} A \Delta
\end{aligned}
$$

Expression (2.17) is an expansion in powers of $\alpha$, where $\alpha \equiv \Delta m_{21}^{2} / \Delta m_{31}^{2}$,

$\Delta \equiv \Delta m_{31}^{2} L / 4 E, A \equiv 2 V E / \Delta m_{31}^{2}, \mathrm{~V}$ is the positive CC potential from the matter effect, $\mathrm{L}$ is the neutrino propagation length, and $\mathrm{E}$ is the energy of the neutrino in GeV. Further derivations and expansions can be found in (18).

To consider antineutrinos or changes in hierarchy, one only needs to identify the parameters in 2.17 that are affected. 'A' will change signs under both charge conjugation and hierarchy swaps. $\delta_{\mathrm{CP}}$ will flip signs under charge conjugation, and $\alpha$ and $\Delta$ will flip signs under changes in hierarchy.

Unlike the MINOS muon neutrino disappearance analysis, which is able to use a twoneutrino formulation approach, the appearance analyses must use (2.17) to account for the increased complexity of the involved physics. It is also important to note the many 
dependencies the expression reveals. This analysis is not completely self-reliant; it depends upon the precision measurements of other MINOS studies as well as those made by other neutrino physics experiments.

\subsection{Summary}

Even against the backdrop of the Standard Model, neutrinos are fascinating particles whose corresponding physical phenomena are worthy of investigation. Fifteen years ago, neutrinos were believed to be massless residents of the standard model; however, the confirmation of neutrino oscillations challenged that notion. In the past year, $\theta_{13}$ has gone from being an unknown parameter to the most precisely measured mixing angle. These advances from reactor-based experiments open the door to further opportunities to answer some of the most fundamental questions about our universe.

In order to answer a few of those questions though, accelerator-based studies are needed, for they provide tunable sources that can produce neutrino and antineutrino beams. Currently, other experiments are being built that aim to measure the $\mathrm{CP}$-violating phase and mass hierarchy. Until then, MINOS can use its appearance analyses to validate the results of the reactor measurements and establish the first limits on the $\delta_{\mathrm{CP}}$-hierarchy phase space. 


\section{Chapter 3}

\section{The NuMI Beam}

The neutrino beam that MINOS utilizes is supplied by the Fermilab complex. The Main Injector is the final accelerator in the chain that delivers protons for the NuMI beam. This device receives protons from the Booster Ring at $8 \mathrm{GeV}$ and feeds protons at $120 \mathrm{GeV}$ to NuMI's graphite target in $10 \mu$ s spills that occur every 2.2 seconds. For a substantial portion of MINOS's running, the proton batches accelerated by the Main Injector were divided between NuMI and the Tevatron. We often define the size of our dataset in terms of protons-on-target (POT), which indicates the exposure delivered to the target - and is proportional to the number of neutrinos produced. Figure 3.1 shows POT as a function of time. The different colors indicate various beam modes.

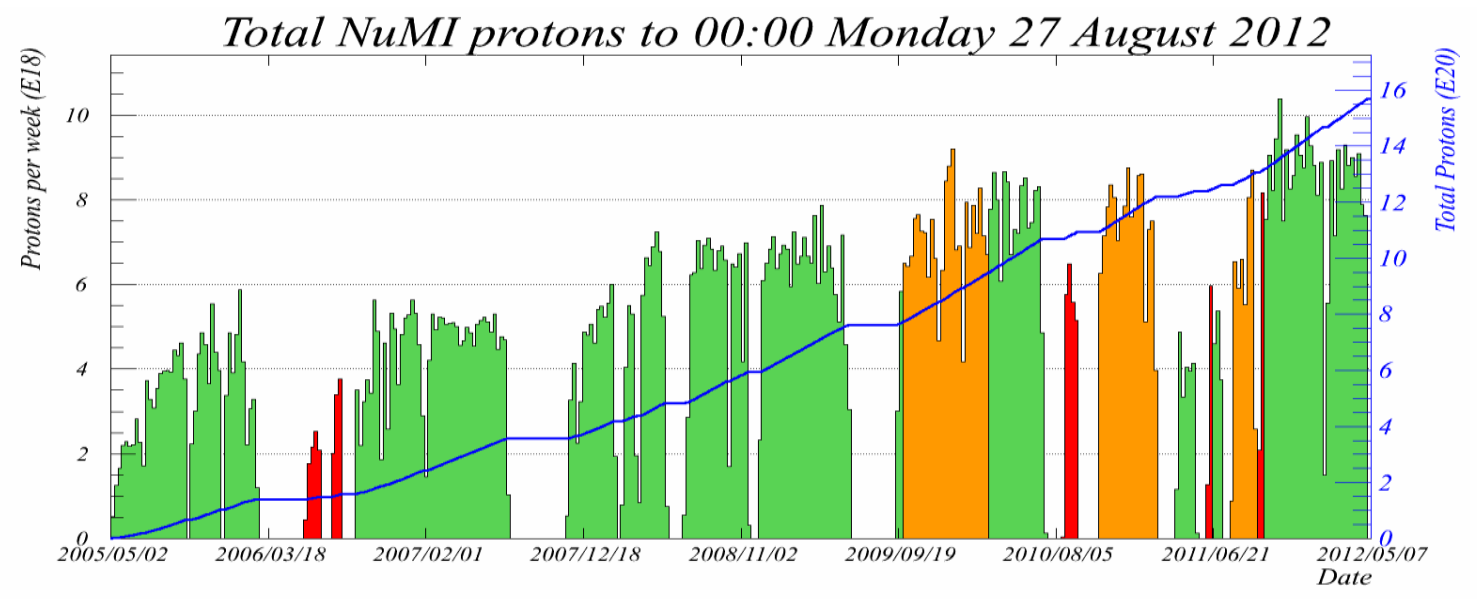

Figure 3.1: The total NuMI protons delivered to the graphite target as a function of time.

Green regions indicate Forward Horn Current (FHC), neutrino mode run periods; orange regions indicate Reverse Horn Current (RHC), antineutrino mode run periods; and red regions designate special intensity run periods. Image taken from (19).

The graphite target resides in the aptly named Target Hall. Incident protons from the Main Injector collide with the target and produce hadronic showers. The target is a 6.4 
$\mathrm{mm} \times 15 \mathrm{~mm} \times 940 \mathrm{~mm}$ rectangular rod that is made up of 48 longitudinal segments called fins. Fins are separated by $0.3 \mathrm{~mm}$, which allows for secondary particles with transverse momentum to escape the target volume without further interaction. Pions and kaons are then free to decay, which enables the production of a neutrinos.

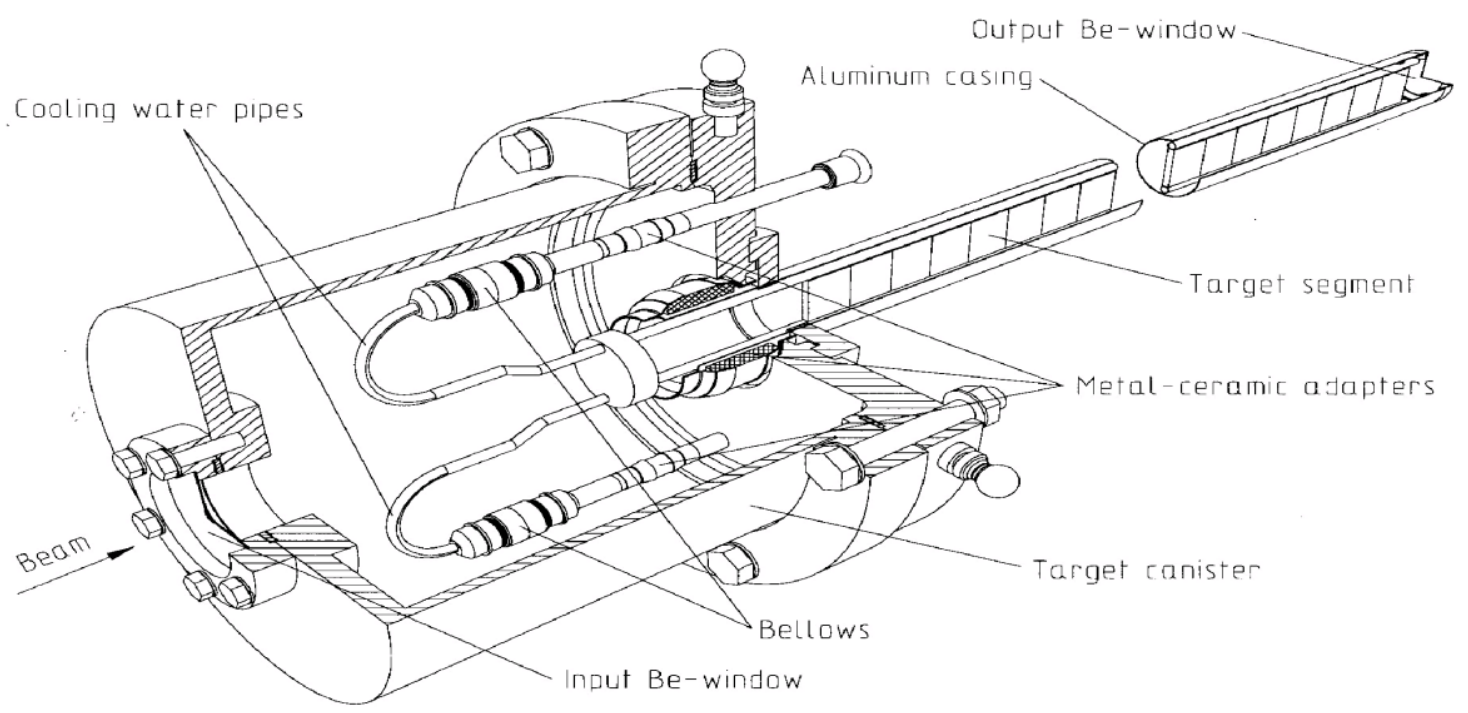

Figure 3.2: Technical drawing of the NuMI target, including its casing and water cooling system. Image taken from (20).

These secondary mesons are produced with a mean transverse momentum of approximately $300 \mathrm{MeV}$. Without a focusing mechanism, the resulting neutrino beam would not be suitable for oscillation measurements due to the lost neutrino flux. Thus, a pair of magnetic horns is implemented to steer off-axis charged particles either towards or away from the beam axis depending on the charge sign.

The horns are made from aluminum and consist of two conducting layers. The outer conductors are cylindrical in shape, whereas the inner layer consists of two back-to-back paraboloids which are separated by a small cylindrical neck. A diagram of the horn design can be seen in Figure 3.3. Axial electric currents of up to $200 \mathrm{kA}$ are pulsed 
through the horns in time with the beam spills. This generates a toroidal magnetic field that focuses secondary mesons of a particular charge, as illustrated in Figure 3.4.

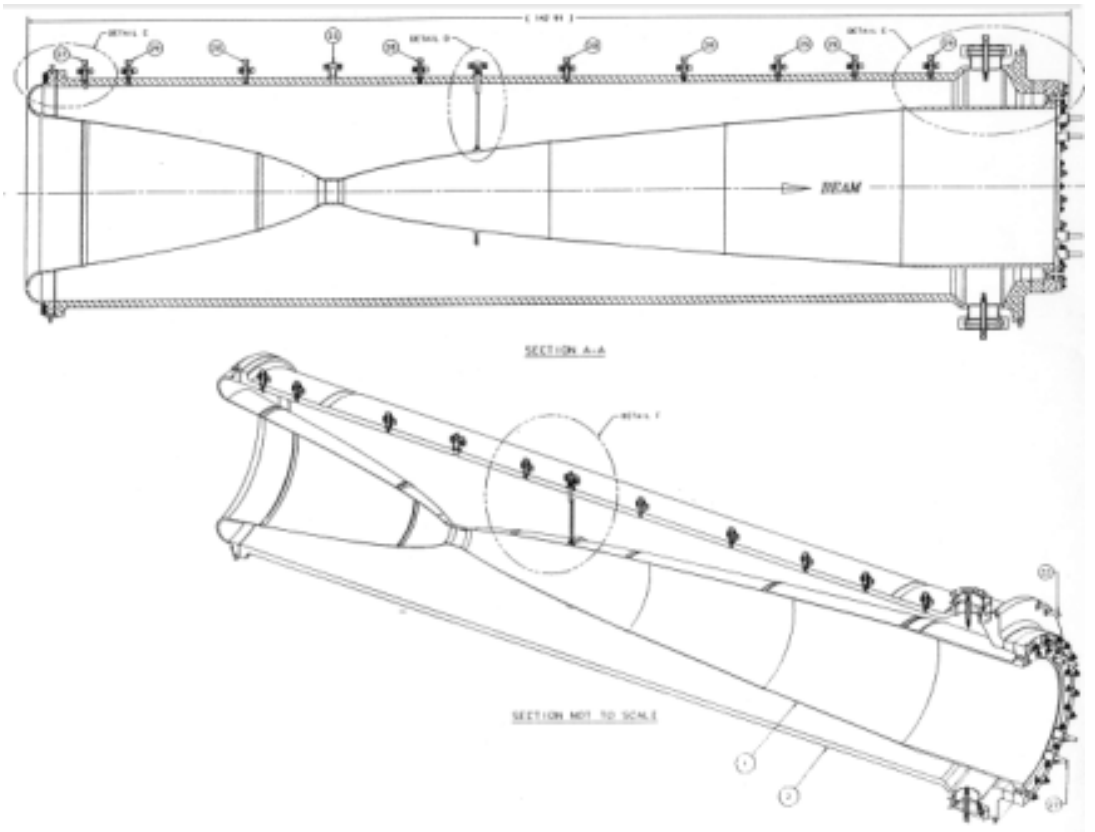

Figure 3.3: Assembly drawing for Horn 2, illustrating the shape and structure of the apparatus. Taken from FNAL drawing 8875.112-ME-363382. (20)

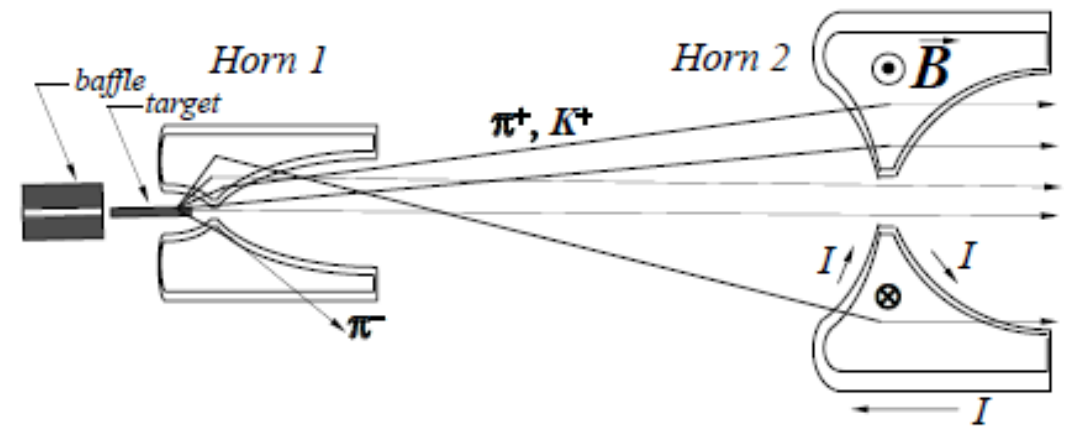

Figure 3.4: An illustration of the sign-selected focusing of secondary mesons produced through proton-target interactions. This particular cartoon illustrates the FHC running mode, in which positively charged particles are focused by the horns to produce a neutrino beam. Image taken from (20).

It is important to note that the focal length of the magnetic horns is proportional to the momentum of the charged particle. By varying the distances between the two horns and the target, as well as the current delivered to the horns, the apparatus can be optimized to 
focus particles of a certain momentum. In particular, NuMI was optimized to focus pions, which produce muon neutrinos in the energy range of interest to observe oscillation. Consequently, the focusing apparatus allows MINOS to tune the energy spectrum of the neutrino beam by steering the pions and kaons that will provide the desired decay products. This feature was useful in the early years of the experiment, when knowledge of the neutrino oscillation parameters was relatively limited.

Given the length of the MINOS baseline and the measured value of $\left|\Delta m_{32}^{2}\right|$, the oscillation maximum occurs at neutrino energies near $1.4 \mathrm{GeV}$. MINOS is capable of running in different energy modes depending upon the setup of the target and focusing horns. As seen in the Figure 3.5, the peak of the 185kA low energy (LE) configuration is closest to the $1.4 \mathrm{GeV}$ peak. Thus, most of MINOS's run time is in the LE- $010 \mathrm{~cm}-185 \mathrm{kA}$ mode.

In addition, beam modes are further divided into two general horn current categories. In the "forward" case, $\pi^{+}$and $\mathrm{K}^{+}$mesons are focused, which results in the production of a neutrino beam; in the "reverse" case, $\pi^{-}$and $\mathrm{K}^{-}$mesons are focused, which results in the production of an antineutrino beam. Data taken in both of these modes will be presented in this thesis. It is the first analysis in which the appearance group has combined data from both neutrino and antineutrino run periods. 


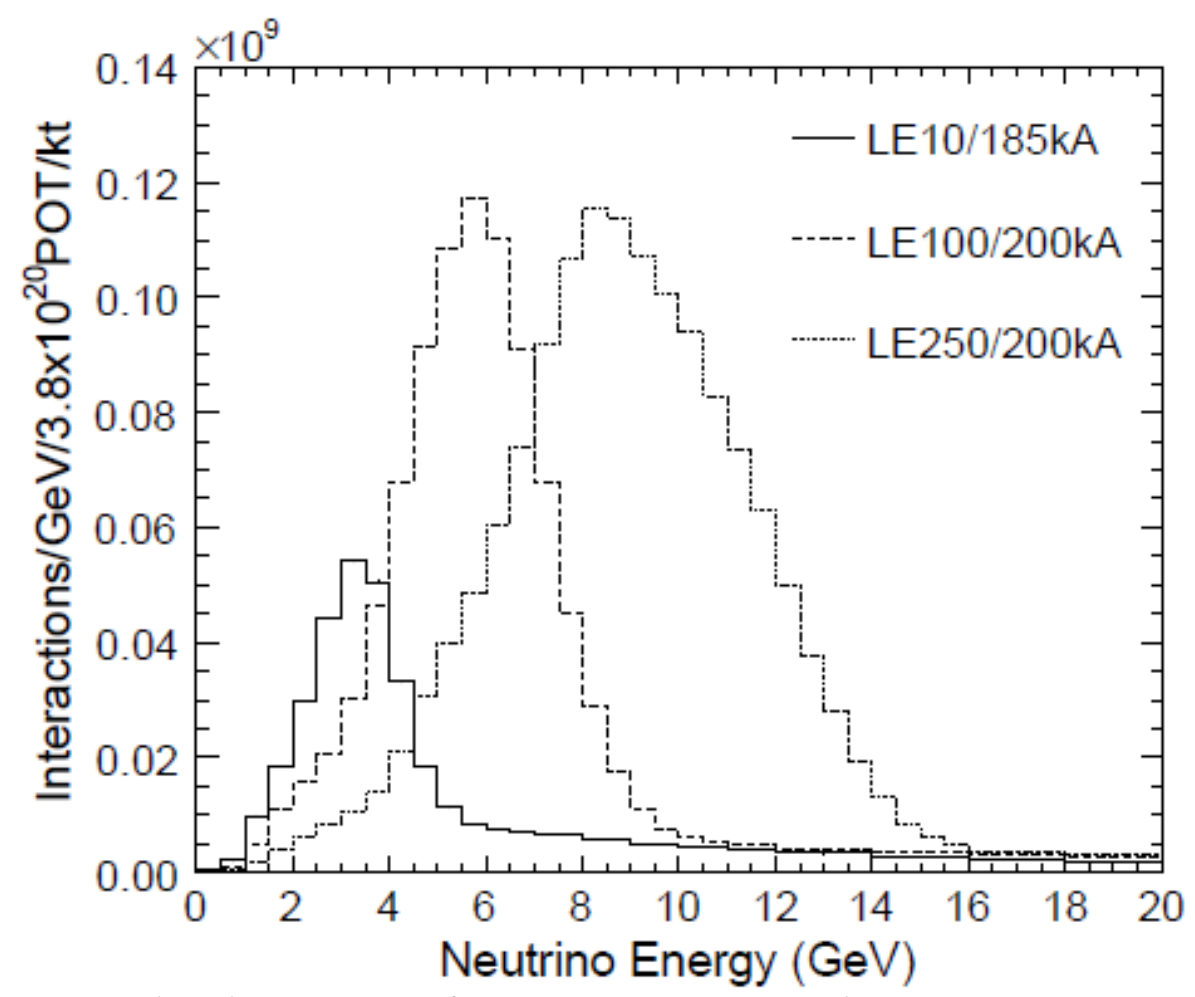

Figure 3.5: Simulated event rate of $v_{\mu} C C$ interactions in the Near Detector as a function of neutrino energy. This comparison of the LE-010 cm-185kA; pseudo-medium energy (pME), LE-100 cm-200kA; and the pseudo-high energy (pHE), LE-250cm-200kA modes demonstrates that the low energy configuration presents the greatest opportunity for MINOS to observe neutrino oscillations. Image taken from (21).

After passing the second horn, secondary particles enter a 675 meter long, 2 meter wide decay pipe. The pipe is capped by two aluminum windows. Originally, the pipe was depressurized to $\sim 1$ torr, but safety concerns prompted the introduction of helium gas into the volume. Inside the tube, mesons are free to drift and decay. In the forward configuration, the beam is primarily composed of muon neutrinos which result from the decay of positively charged pions. Tertiary muons formed in this interaction can also decay weakly, which produces a neutrino-antineutrino combination that contributes to the overall NuMI flux. 
Hadrons that do not decay in the pipe are stopped by a hadronic absorber placed immediately downstream from the end of the pipe. The absorber consists of a watercooled aluminum block, steel shell, and concrete backstop. Any tertiary muons that escape the decay pipe are not impeded by the hadronic absorber. To screen out the unwanted muons, the beam is directed through 240 meters of unexcavated rock. The muons dissipate their energy by ionization as they travel through the material, but the weakly interacting nature and neutral charge of neutrinos allows those particles to pass unimpeded. Muon monitors interspersed along the beamline measure the alignment and spread of the NuMI beam upstream from the Near Detector. Figure 3.6 provides an illustration summarizing the entire production chain.

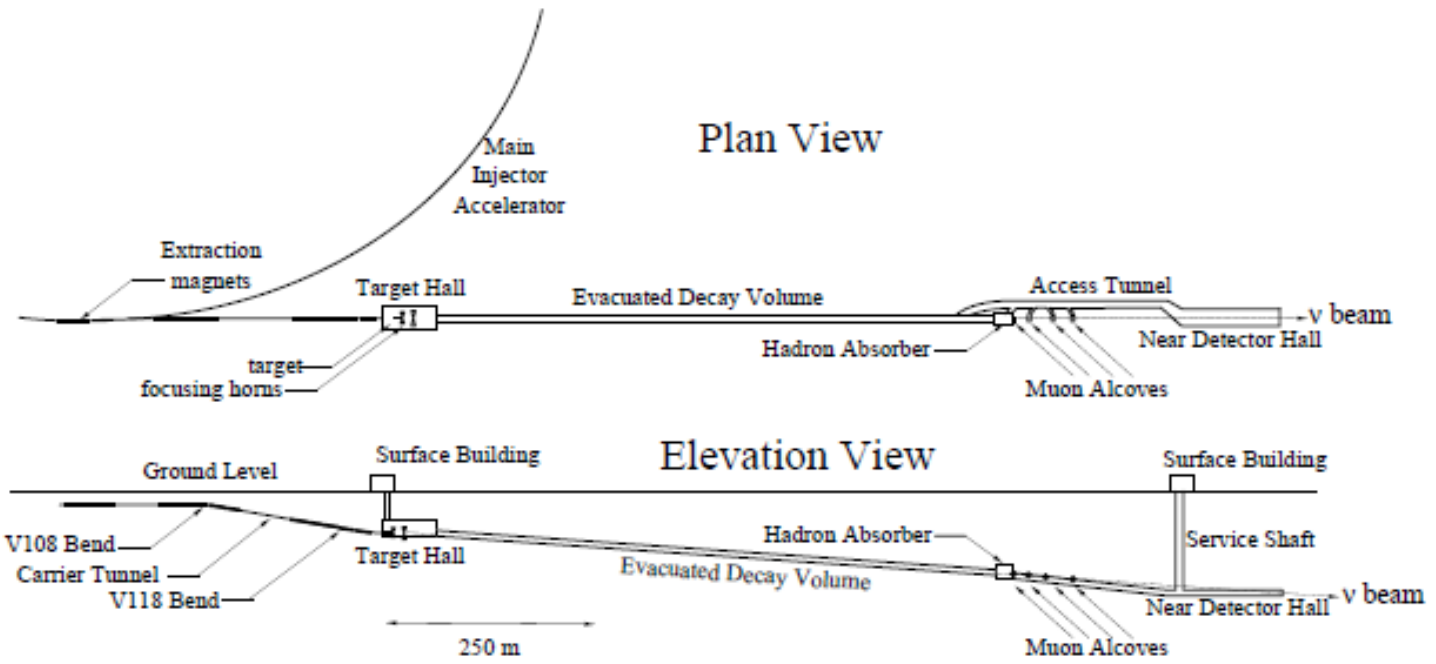

Figure 3.6: Plan and elevation views of the NuMI beam facility. This illustration presents the overall picture of the production chain from proton injection to neutrino beam creation. Image taken from (21).

The beam's neutrino flavor compositions for the forward and reversed horn current modes are measured at the Near Detector. For the forward case, the $v$-enhanced beam is composed of $91.7 \% v_{\mu}, 7.0 \% \bar{v}_{\mu}$, and $1.3 \% v_{e}+\bar{v}_{e}$. In the reverse case, the $\bar{v}-$ enhanced 
beam is composed of $58.1 \% v_{\mu}, 39.9 \% \bar{v}_{\mu}$, and $2.0 \% v_{e}+\bar{v}_{e}{ }^{(22)}$ While there is a substantial contamination of muon neutrinos in the antineutrino beam mode, the energy spectrum of these particles is much broader than the antineutrino distribution. In addition, the maximum of the contaminant distribution occurs at approximately $9.2 \mathrm{GeV}$, which is outside the optimal energy range for observing antineutrino oscillations.
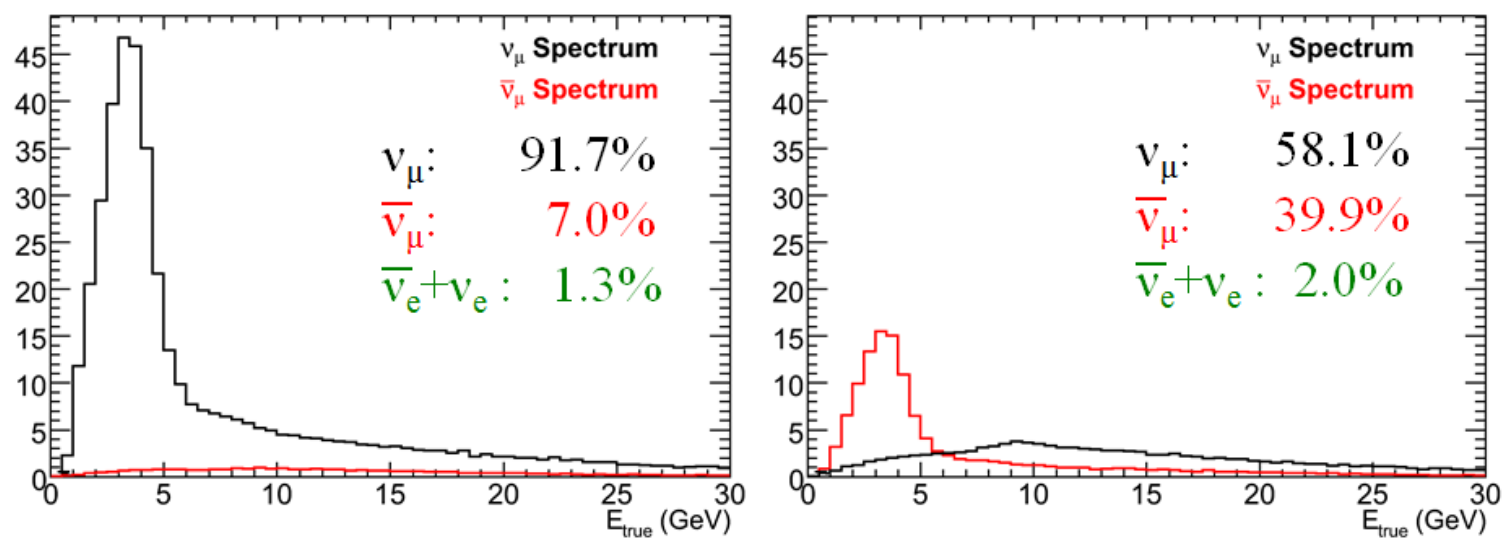

Figure 3.7: Energy spectra of neutrino events in the Near Detector in both the neutrino mode (left) and antineutrino mode (right). The y-axis is an arbitrary event rate scale to which both figures have been normalized. Image taken from (23). 


\section{Chapter 4}

\section{The MINOS Experiment}

\subsection{General Detector Characteristics}

MINOS employs two functionally-identical steel-scintillator calorimeters to measure the energy depositions and topologies of neutrino candidate events. The detectors are separated by 734 kilometers, the first being located on the Fermilab grounds $1 \mathrm{~km}$ downstream from the graphite target and the second being located in the Soudan Mine in Northern Minnesota.

Each detector consists of alternating layers of $1.00 \mathrm{~cm}$ thick polystyrene plastic scintillator and $2.54 \mathrm{~cm}$ thick steel. The steel planes, which are magnetized by coils aligned to the longitudinal axes of each detector, represent the vast majority of the detector masses. They serve as the primary targets for neutrinos in the NuMI beamline, which interact with the iron nuclei to produce the particle tracks and showers that are observed thanks to the scintillator regions. The magnetic fields produced by the coils facilitate charge-sign identification and momentum determination of muons that travel through the detectors.

Each $4.1 \mathrm{~cm}$ wide scintillator strip is coated with a titanium dioxide layer; which aids the light yield, helps optically isolate each strip, and offers a little protection from accidental damage. Adjacent layers of scintillator are oriented in an orthogonal arrangement to facilitate 3-dimensional track reconstruction. The arrangements are defined along $U$ and V axes; hence, planes are also categorized by the particular alignment of their strips. Wavelength shifting (WLS) fibers, sealed with a reflective adhesive strip into an 
indentation extruded along the top-center of each strip, absorb the light generated by incident ionizing particles interacting with the scintillator. The WLS fibers are $1.20 \mathrm{~mm}$ in diameter and are double-clad polystyrene cords doped with Y11 fluor to $175 \mathrm{ppm}$. To prevent self-absorption, the fibers were made to absorb light with wavelengths around $420 \mathrm{~nm}$ and re-emit light at $470 \mathrm{~nm}$.

Signals are carried by the WLS fibers to the readout system, which consists of multianode photomultiplier tubes (PMTs). To shield the PMTs from light, magnetic fields and electronic noise, each PMT is stored in a light-tight box. 194 64-anode Hamamatsu PMTs are used at the Near Detector and each PMT is housed in its own enclosure. At the Far Detector, 1452 16-anode Hamamatsu PMTs are used and each enclosure contains three photomultiplier tubes. Energy deposition signals received by the PMTs are digitized, and the data acquisition (DAQ) system records the time of each hit, the channel in which each hit occurred, and the pulse height of each hit in ADC units. The DAQ systems of the detectors are functionally-identical to minimize potential systematic errors that could arise from using vastly different systems.

Combining the information supplied to the DAQ, the topologies and energies of events can be reconstructed. Figures 4.1-4.3 illustrate the general structure of the MINOS detectors, the polystyrene scintillator, and the readout system. While these systems were designed to be as similar to one another as possible, there are differences between the Near and Far Detector systems, and purposes, that warrant further explanations. 


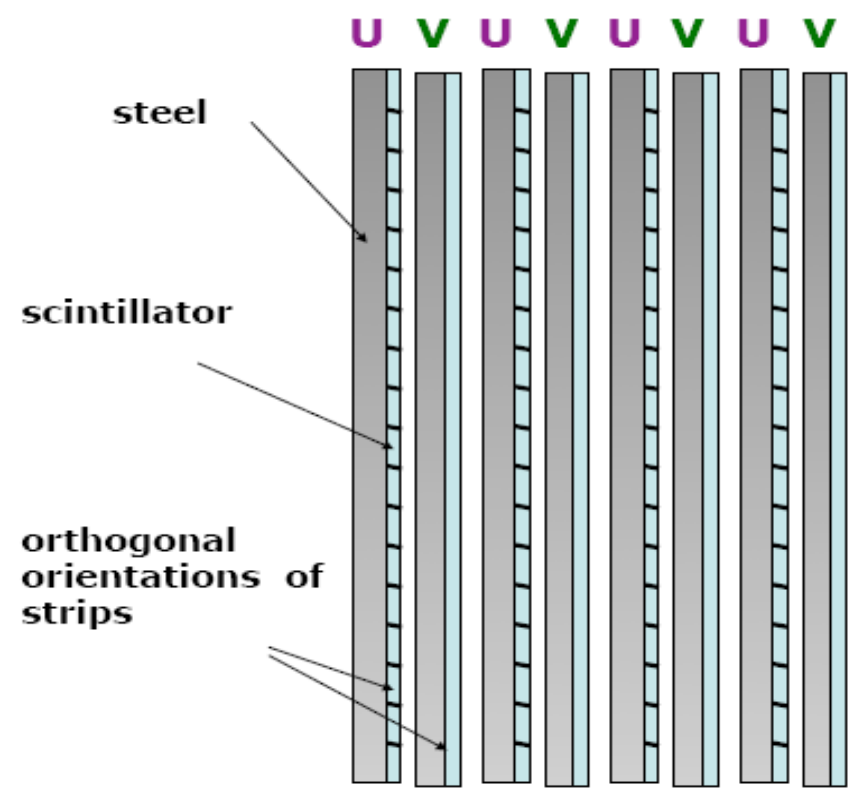

Figure 4.1: Illustration depicting the MINOS steel and scintillator planes. Note the orthogonal orientation of adjacent scintillator layers. Image taken from (24).

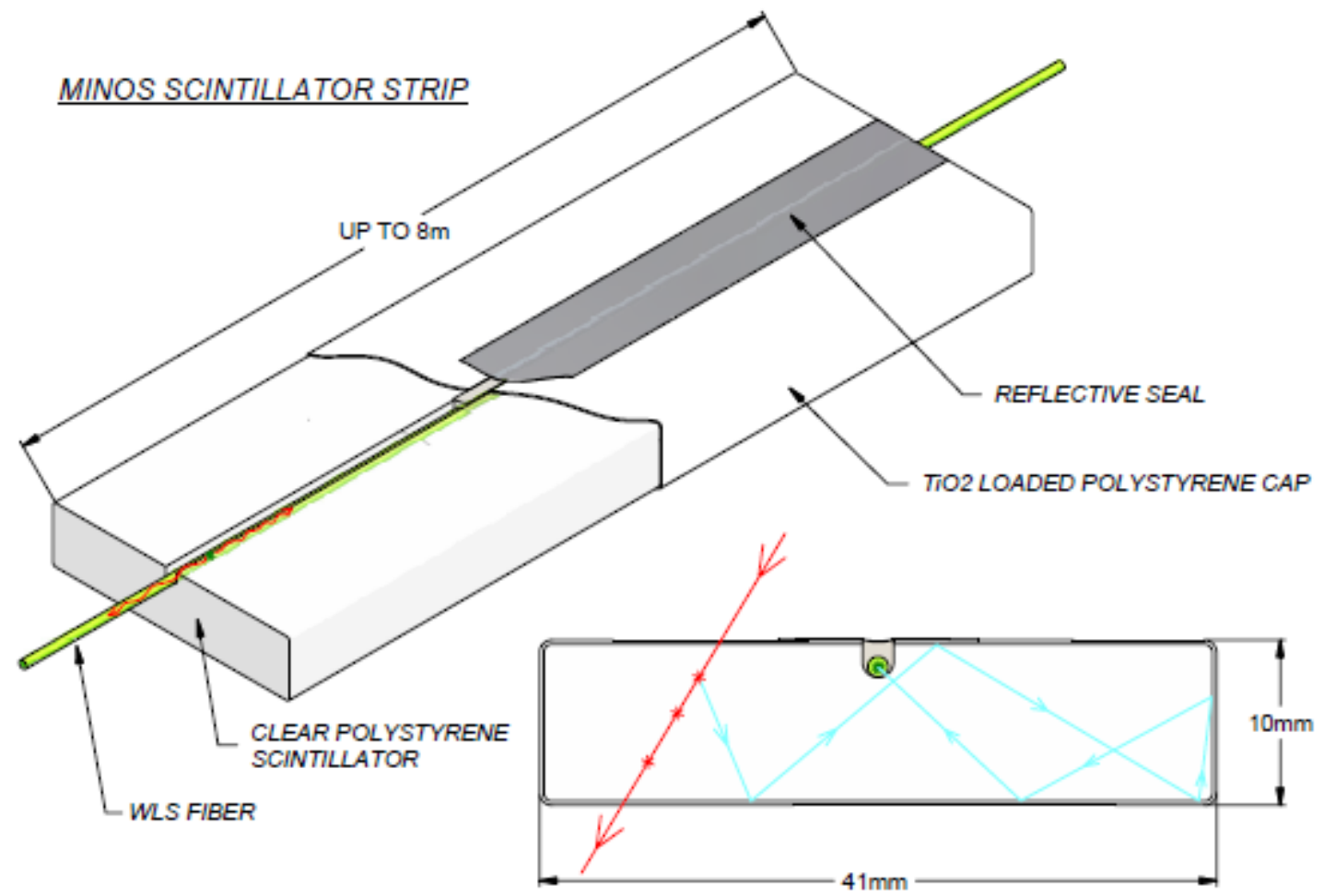

Figure 4.2: A strip of MINOS scintillator with the WLS fiber installed along the central groove. The cutaway in the lower right corner of the image depicts an incident charged particle (red line) that produces light (blue line) as it passes through the strip. This light is ultimately transmitted to the PMTs by the WLS fiber. Image taken from (25). 


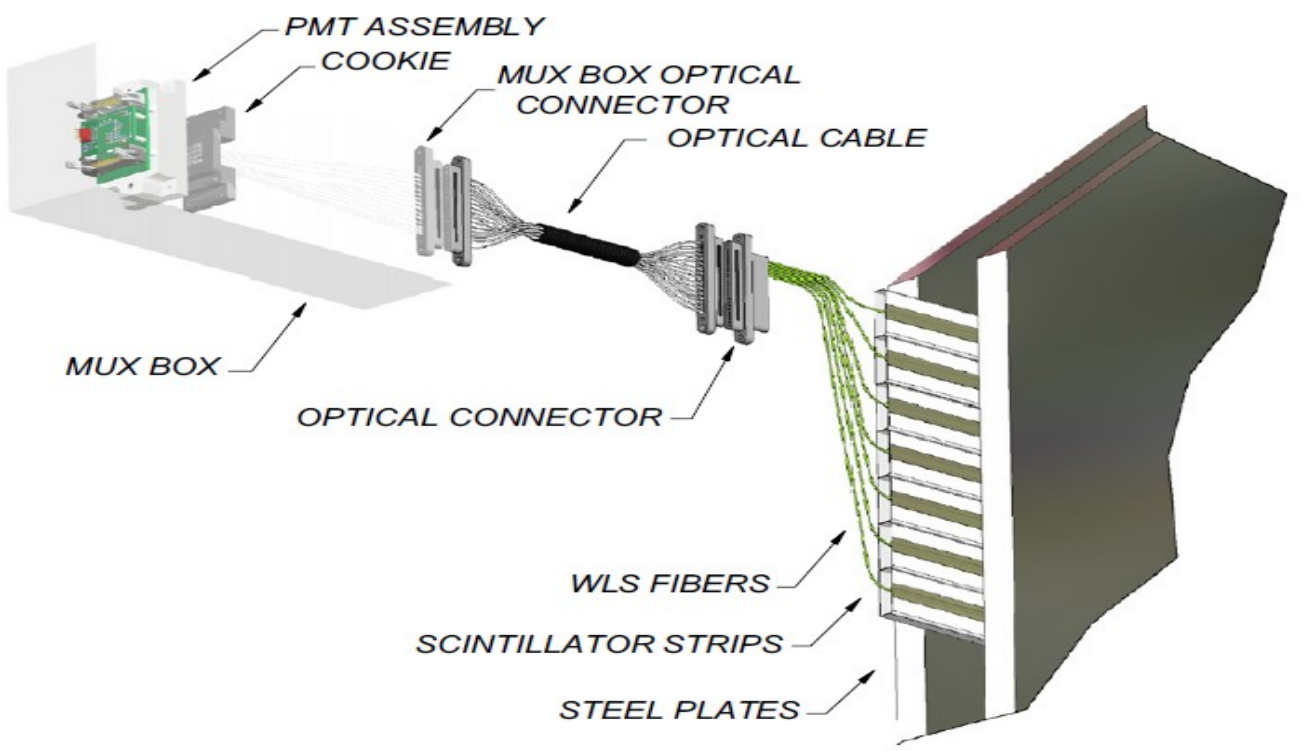

Figure 4.3: Diagram of the readout assembly of the MINOS detectors. Light produced in the detector is carried to a clear optical cable by the WLS fibers. The cable carries the signal into the MUX box (PMT enclosure) and to the PMT. Image taken from (25).

\subsection{The Near Detector}

Neutrino interactions in the Near Detector are used to determine the composition of the NuMI beam before significant propagation has occurred to enable flavor oscillations. The detector is located about $1 \mathrm{~km}$ from the graphite target in an excavated cavern on the Fermilab site. The detector sits roughly 100 meters below the Earth's surface, and the rock above provides a 225 meters-water-equivalent (mwe) depth.

Due to the proximity of the Near Detector to the neutrino source and the intensity of the NuMI beam, many neutrino interactions are recorded by this apparatus. On average, 16 neutrino interactions occur in the detector during each $10 \mu \mathrm{s}$ beam spill, which amounts to nearly 630,000 interactions per day, assuming $100 \%$ beam uptime. This necessitated the development of front-end electronics that could cope with the large number of hits registered by the detector. Special circuit boards, called MENUs, were created to serve 
this purpose. Each MENU is connected to one of a PMT's 64 pixels and contains an analog-to-digital converter, a charge integration encoder (QIE), and a data buffering system. MENUs are grouped onto larger circuit boards called MINDERs, which are further clustered into modular readouts called MASTERs. Hits passed to the MASTERs are channel-tagged and time-stamped for analysis.

282 planes make up the detector, which has a mass of 980 metric tons. The steel plates of each plane are 6.2 meters wide by 3.2 meters tall and take an irregular octagonal shape. The strength of the magnetic field in the detector, averaged over the fiducial volume, is 1.2 $\mathrm{T}$, and the coil that produces this field is situated away from the center of each plane to produce a fiducial region that is aligned with the beam.

Scintillator planes of the Near Detector are either partially instrumented (only covering the fiducial region) or fully instrumented (covering the fiducial region as well as the area around the coil hole). Figure 4.4 provides a general schematic of the plane configurations, and Figure 4.5 illustrates the fiducial volume of the detector. 

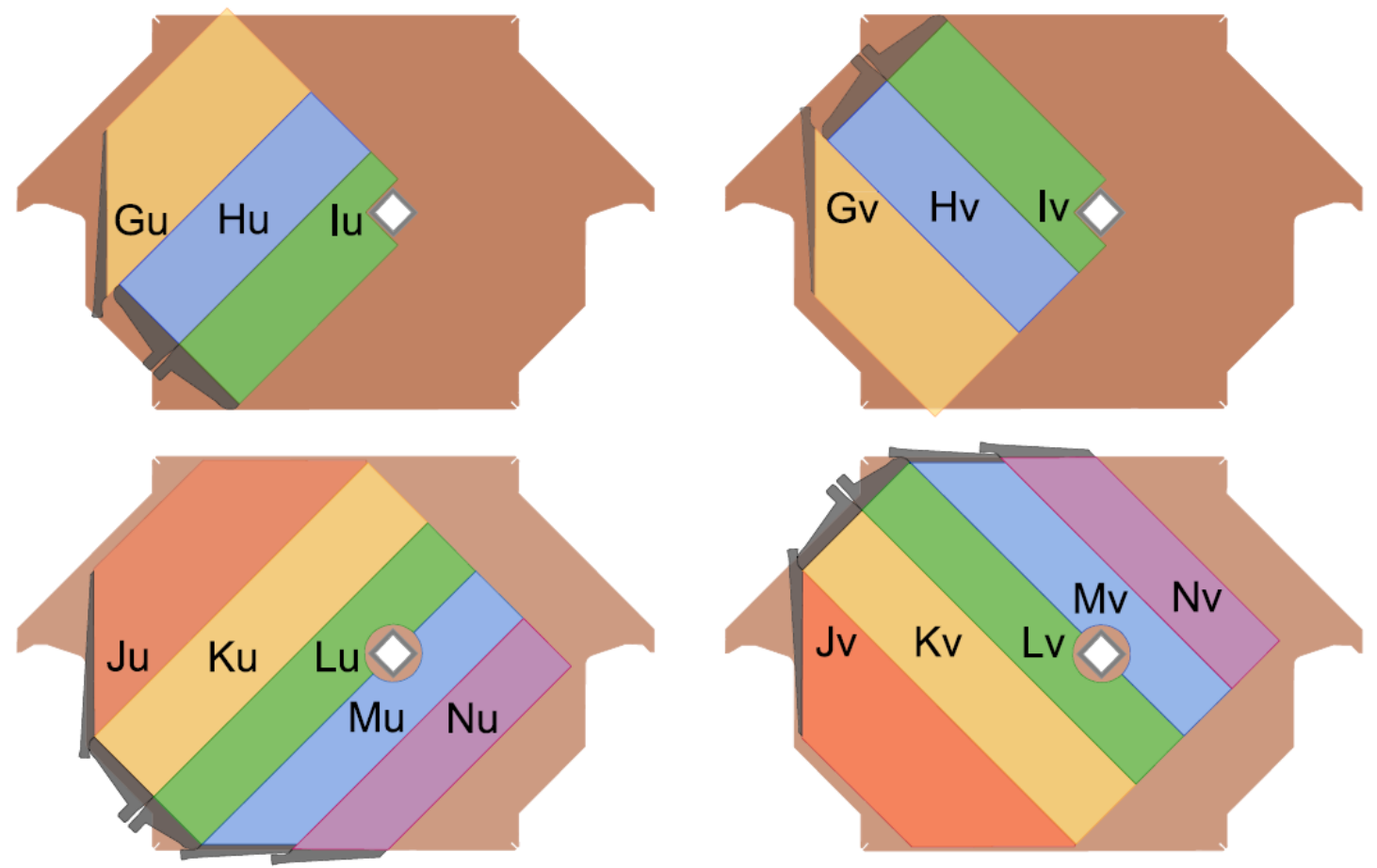

Figure 4.4: Configurations of Near Detector scintillator planes. The tan region represents the silhouette of the steel planes in the detector. The top two images represent partially instrumented planes, whereas the bottom two images are fully instrumented. $U$ strip orientations are on the left, and V strip orientations are on the right. Strips are grouped together in modules, designated by the $G$-N lettering scheme of the colored bands. Image taken from (25). 

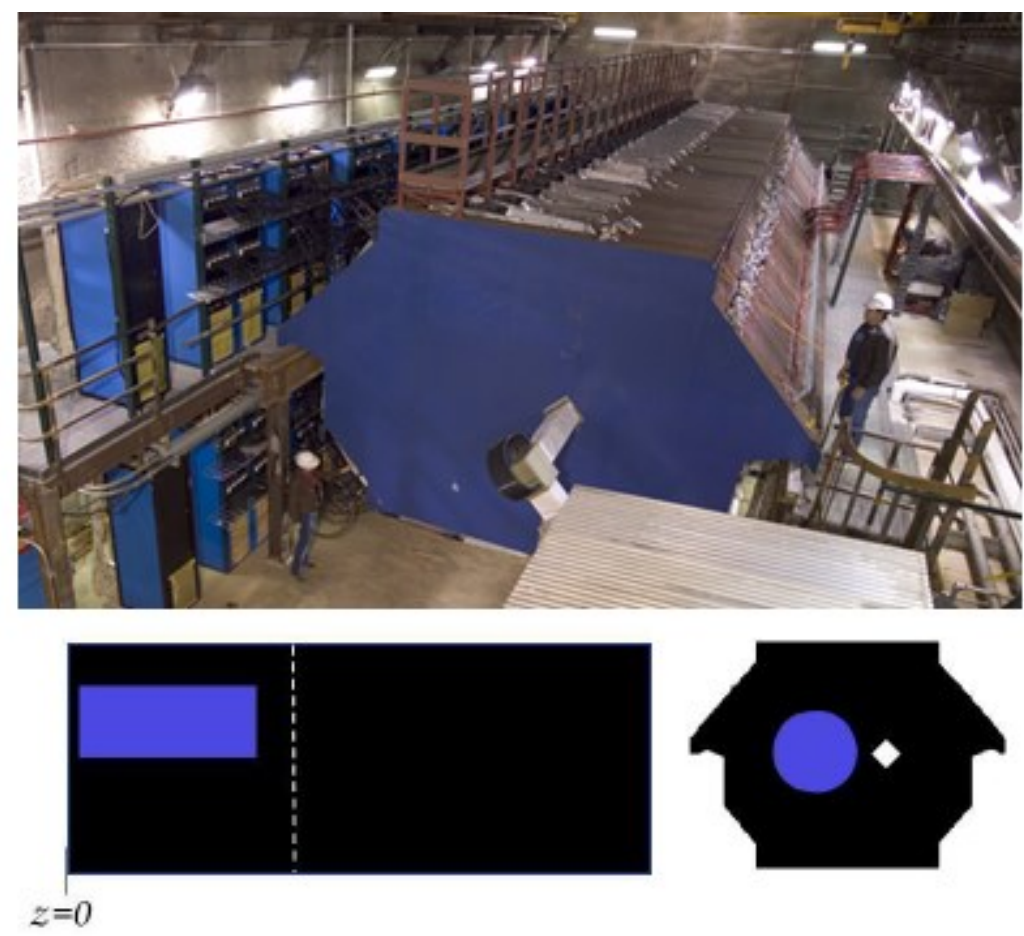

Figure 4.5: Picture of the Near Detector located at Fermi National Accelerator Laboratory. The cartoon beneath the image illustrates the fiducial region of the detector in blue. The white diamond represents the coil hole. Image from (26).

Furthermore, the detector is divided into calorimeter and spectrometer parts, both of which serve distinct functions and use different assortments of scintillator planes.

Situated closer to the graphite target, the calorimetric region makes precision measurements of the topologies and energies associated with neutrino interactions. Every fifth scintillator plane in this section is of the fully instrumented variety, whereas the other four in each group are only partially instrumented. All 120 planes that make up the Near Detector calorimeter are read out by the Hamamatsu PMTs.

Behind the calorimetric region, the Near Detector spectrometer tracks muons, particularly those created via charged current interactions. Like the calorimeter, every fifth scintillator plane is fully instrumented; however, these are the only planes read out by photomultiplier tubes, and there are no partially instrumented planes in this section. 
Considering the attenuation length of the WLS fibers and the size of the Near Detector, we are able to read out light from only one end of the scintillator strips. In the calorimeter region of the detector, each WLS fiber is covered by an individual PMT pixel. In the spectrometer section, four WLS fibers are tied to a PMT pixel to reduce the number of necessary components. This is possible thanks to the broad spread and specific function of the spectrometer, for one pixel can still be used to cover multiple strips without incurring losses in reconstruction power.

Two triggering modes are used by the DAQ system at the Near Detector that handle two distinguishable event types. For NuMI-related neutrino interactions, a time-based "spillgate" trigger is utilized. This trigger continuously digitizes hits in the detector during a time window that starts $1.5 \mu$ s before the beam spill begins and lasts for $13 \mu \mathrm{s}$. Cosmic ray data is acquired through a PMT activation threshold trigger called the "dynode mode".

\subsection{The Far Detector}

The function of the Far Detector is to observe neutrino interactions after neutrinos from the Fermilab site have had sufficient time to undergo flavor oscillations. The detector sits 705 meters underground in an old iron mine in Soudan, Minnesota, where the rock above provides an overburden of nearly 2100 mwe. The detector is 753.3 kilometers from the NuMI target. Hence, it experiences a significantly reduced flux compared to that in the Near Detector. It was due to this fact that the site was selected, as the overburden shields the detector from most cosmic ray muons and other surface sources of radiation. The Far Detector is substantially larger than its Near counterpart. Each plane of the 
detector is an $8 \mathrm{~m}$ wide regular octagon, and the total mass of the detector is 5.4 kilotons. Unlike the Near Detector, scintillator strips in the Far Detector cover the entire surface area of a plate to maximize event reconstruction abilities. The Far Detector is divided into two supermodules, each of which possesses its own magnetic coil that runs axially down the geometric center of the detector planes. The first supermodule consists of 249 planes and is 14.78 meters long, while the second supermodule consists of 237 planes and is 14.10 meters long. Averaged over the fiducial volume, the magnetic field provided by the coils is 1.42 T. Figure 4.6 shows a general depiction of the Far Detector, and Figure 4.7 illustrates the detector's fiducial region.
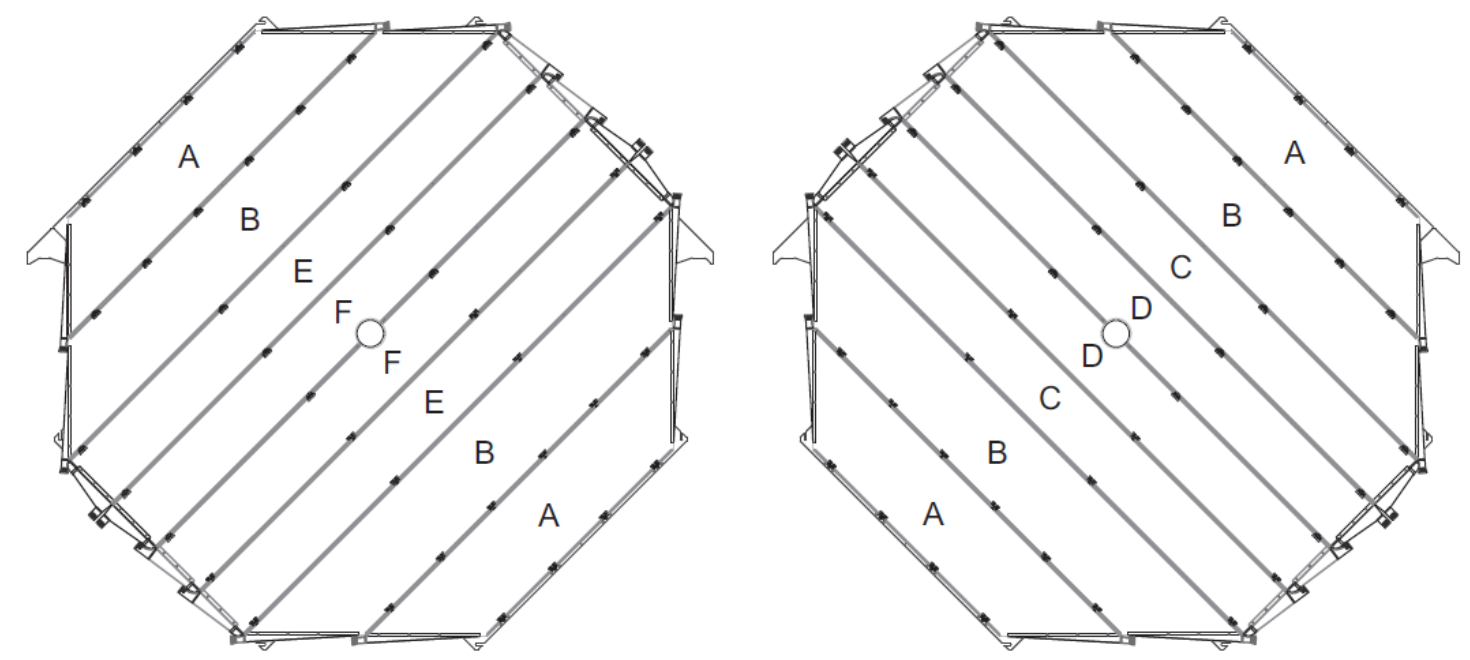

Figure 4.6: Depiction of the plane configurations of the far detector. The U-type plane is shown at left and the V-type plain is shown at right. Once again, strips were clustered together into modules, which are represented by the lettered bands in the image. A and B-type modules contain 28 scintillator strips; the others contain 20. Image from (25). 


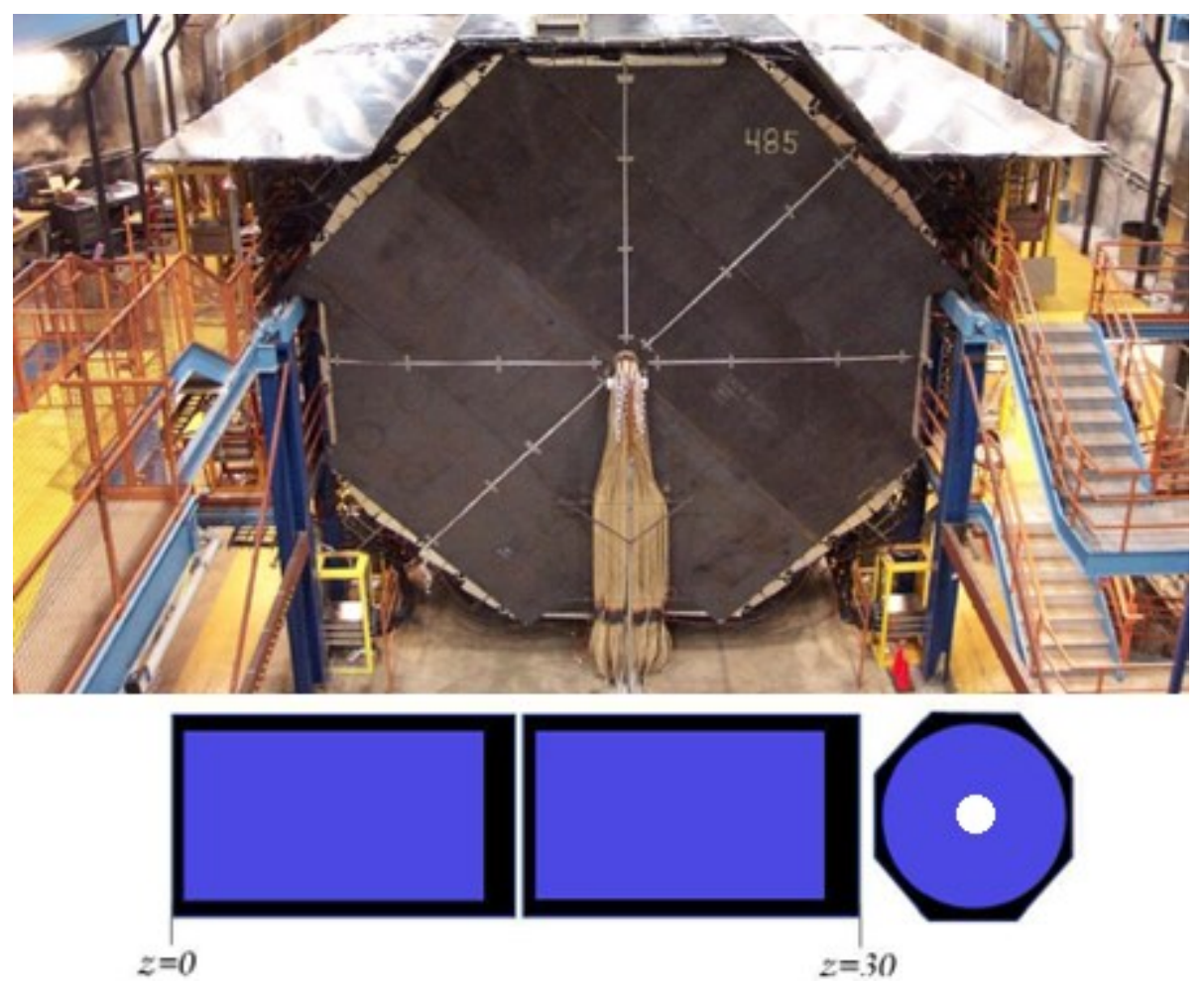

Figure 4.7: Picture of the Far Detector located in the Soudan Mine in Northern Minnesota. The cartoon beneath the image illustrates the fiducial region of the detector in blue. The white circle represents the coil hole. Image from (26).

WLS fibers in the Far Detector are read out from both ends of the scintillator strips. Just like the spectrometer region of the Near Detector, multiple fibers are tied to an individual PMT pixel. In this case, eight fibers, separated by at least 1 meter are optically connected to a pixel, and an algorithm is used to properly reconstruct the true locations of strip hits. Once again, this technique minimizes the number of electronic devices needed and reduces the effects of PMT crosstalk.

Commercially available front-end electronics were selected to handle the readout system because the Far Detector registers only a few NuMI-related events per day. In every light-tight box, there are three VA chips that accept signals from each of the PMTs. These chips are mounted on front-end boards called VFBs, which power and bias the 
VAs and initiate the readout chain. Two VFBs deliver their analog signal outputs to a VA Mezzanine Module (VMM), which houses an analog-to-digital converter. Six VMMs are controlled by a VA Readout Controller (VARC), which is responsible for the digitization of hits, the time-stamping of events observed in the detector, and triggering of the VA chips.

The DAQ system at the Far Detector is triggered by two different mechanisms. A GPS system signals that protons have been delivered to the NuMI target. Hits in the Far Detector are then automatically digitized, and hits in the detector are digitized in a $100 \mu \mathrm{s}$ window concurrent with the beam spill. In addition, several PMT activation thresholds are introduced. Triggering will occur if 4 out of 5 adjacent planes register strip hits, if 4 adjacent planes reach a 20 PE summed threshold, or if a total of at least 20 planes record interaction activity. This $2^{\text {nd }}$ trigger mode enables analysis of atmospheric neutrino events and events induced by cosmic ray muons.

\subsection{Veto Shield}

To further improve the rejection of cosmic ray background events that could interfere with the Far Detector's ability to register signal candidates, a veto shield was constructed at the Soudan site. It completely covers the top of the detector and offers partial coverage of the detector's east and west sides. The shield is constructed using three layers of the experiment's standard scintillator strips, which are aligned longitudinally with the Far Detector. PMTs read out both ends of the strips, with eight adjacent fibers being optically connected to a single pixel. 


\subsection{The Calibration Detector}

A 12-ton Calibration Detector (CalDet) was built at CERN using 60 one meter square planes of steel and scintillator. Unlike the other detectors, the CalDet possesses no magnetized steel plates. Both Near and Far Detector electronics were connected to the Calibration Detector to measure their responses to controlled test beams of electrons, muons, pions and protons. Topological and calorimetric data collected from CalDet were used to ascertain the absolute calibrations MINOS would use in future analyses.

\subsection{Event Types and Reconstruction}

Before we can uncover any enlightening physics, we need to develop the ability to reconstruct neutrino candidates from the raw data collected from the detectors. This amounts to converting ADC counts, strip hit position and timestamps into particle showers and tracks. Once this information is extracted, MINOS can identify an event's type and use the accompanying calorimetric and tracking data to measure relevant oscillation parameters. Therefore, the primary goal of the reconstruction system is to classify the event type and determine the energy of the neutrino related to that event.

\subsubsection{Event Types}

The MINOS detectors were optimized to search for muon neutrinos that remained in the NuMI beam to ascertain the survival probability of the $v_{\mu} \rightarrow v_{\tau}$ transition. This particular oscillation mode is studied by selecting $v_{\mu} \mathrm{CC}$ candidates from the raw data. In this charged current interaction, the incoming muon neutrino exchanges a $\mathrm{W}$ boson with a target nucleus (most likely contained in the massive steel planes). The hallmark signature of this event is a long muon track, which extends beyond the hadronic shower activity of the nuclear recoil. In this regard, reconstructing $v_{\mu} \mathrm{CC}$ events is a relatively simple task. 
The situation pertaining to $v_{\mathrm{e}} \mathrm{CC}$ events in MINOS is more complex. Electromagnetic showers in MINOS have an average radiation length of $4.06 \mathrm{~cm}$ and an average Molière radius of $3.7 \mathrm{~cm}$. Comparing these numbers to the plane separation of $5.95 \mathrm{~cm}$ and the strip width of $4.1 \mathrm{~cm}$, we note that MINOS does not have the power to resolve an electron or positron track. Instead, identification algorithms must have the ability to pluck the electron/positron characteristics from a shower that overlaps hits from the hadronic recoil system. The development of these algorithms and their implementation represent a substantial portion of the work related to this thesis, and these techniques will be presented in greater detail in Chapter 6.
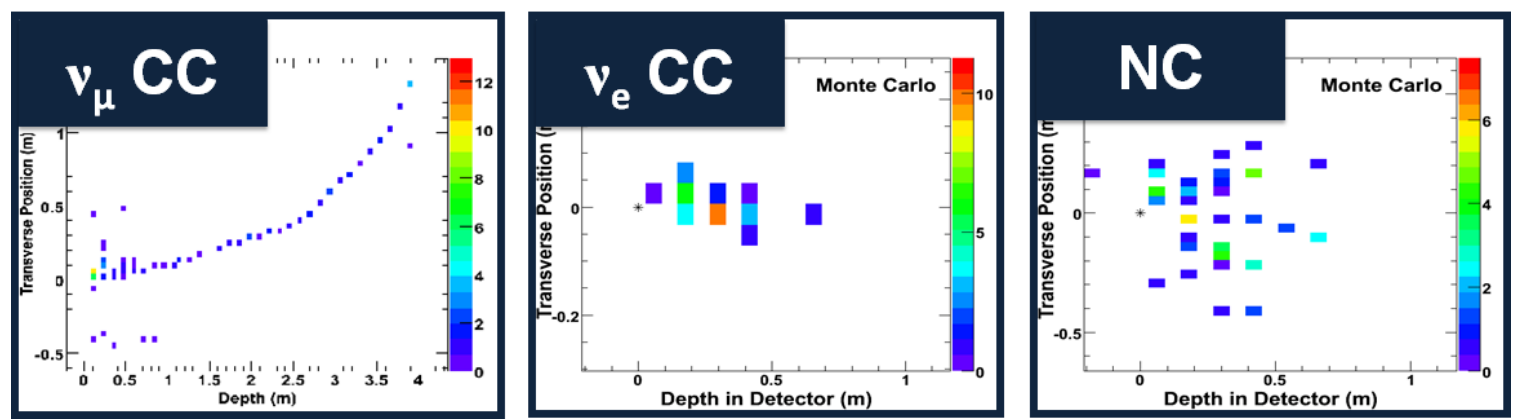

Figure 4.8: Monte Carlo event displays of $v_{\mu} C C$ (left), $v_{e} C C$ (center), and NC (right) interactions in the MINOS Far Detector. Note the long muon track present in the left image compared to the compact natures of the electron neutrino and neutral current events. The similarities between the topologies of $v_{e} C C$ and NC interactions in the detector necessitated the implementation of a statistical selection method. Image from (27).

The most prominent background in the appearance analyses is supplied by neutral current events, in which an incoming neutrino will exchange a $\mathrm{Z}$ boson with a target nucleus. In this case, only the hadronic recoil system is seen in the detector as a charged lepton was not produced. Typically, these showers are more diffuse than their electromagnetic counterparts, but there are several instances in which $\mathrm{NC}$ events can be particularly problematic for the appearance searches. $\mathrm{NC}$ events may contain electromagnetic shower 
components if the products of the interaction contain a $\pi^{0}$. This makes it far more difficult to tag the events as neutral current interactions and separate them from the $v_{\mathrm{e}} \mathrm{CC}$ signal. In addition, since the detector only responds to the hadronic shower and does not include the exiting neutrino, the reconstructed energy of shower only represents a portion of the incident neutrino's true energy. In this manner, it is possible that a high energy neutrino well outside the oscillation energy range will produce a hadronic shower that contaminates the signal region upon reconstruction.

Beam $v_{\mathrm{e}} \mathrm{CC}$ events must also be considered by the appearance analyses. These events consist of electron neutrinos or antineutrinos which come directly from the contamination components of the NuMI beam. As these events are topologically identical to our signal events, we predict the number of beam $v_{\mathrm{e}}$ events expected at the Far Detector given the number of protons-on-target.

In addition to the above interactions, $v_{\tau} \mathrm{CC}$ events also warrant some discussion. Since the dominant transition in $v_{\mu}$ disappearance is $v_{\mu} \rightarrow v_{\tau}$, there is a possibility that a tau particle could interact with the Far Detector if the incident neutrino energy was greater than the $3.55 \mathrm{GeV}$ threshold. These interactions have not been identified in MINOS at this time, but as their decays produce electrons or hadronic showers, the expected background contribution from this interaction is calculated.

\subsubsection{Reconstruction}

As mentioned previously, the aim of reconstruction is to convert the information collected by the MINOS detectors into physically meaningful entities that can be analyzed by the MINOS Collaboration. This goal is achieved by ascertaining the topology an event and by deducing the visible energy associated with an interaction. 
In the Near Detector, it is common for a handful of neutrino interactions to be observed in a single beam spill. The hits recorded in a single spill are called a snarl, and the first job of reconstruction is to disentangle individual events from the diffuse mess.

Interactions are separated by considering the location of hits in the detector and the timestamp attached to each energy deposition. Events at the Far Detector are also divided into snarls to match the process at the Near Detector; however, due to the reduced flux, FD snarls contain zero or one neutrino interaction.

Once the collection of interactions has been separated into individual slices, a trackfinding algorithm examines each event. Segments of a track are first identified and then chained to other found segments to construct the full track. Once the complete track has been reassembled, a Kalman Filter is used to fit for the trajectory curvature. If a muon track does not end within the detector volume, its curvature under the magnetic field is used to determine the muon's momentum. In the case where the track ends inside the detector, the track's range is used to do the momentum calculation. The muon momentum resolution is $5 \%$ for the track-range method and $10 \%$ for the track-curvature method. Reconstructing showers necessitates a slightly different approach because the granularity of the detector is insufficient for tagging individual particle tracks amongst the shower constituents. Instead, reconstruction uses hits that are localized in space, timestamped accordingly, and were not tagged by the track-finding algorithm. The energy of the shower is calculated by summing the pulse heights of the individual hits. In the case energy is deposited into a strip by both a shower and a track, the estimated pulse height imparted by the track is subtracted from the shower sum. 
Figure 4.8 shows Monte Carlo event displays for the different event topologies seen in the MINOS detectors. The characteristic track of the $v_{\mu} \mathrm{CC}$ event is easily spotted. The key point to take away from the $\mathrm{NC}$ and $v_{\mathrm{e}} \mathrm{CC}$ illustrations is that these events can appear to be similar in both shape and energy deposition.

\subsection{Calibration}

MINOS employs two calibration chains to assist the reconstruction effort in returning accurate energy calculations from the detector response. The first, called the Energy Branch, is used to convert raw ADC counts into deposited energy. The second, called the Photoelectron Branch, takes the signal and converts it into photoelectron units. A flow chart in Figure 4.9 outlines the two paths. This calibration is particularly important to the appearance analyses, which require precise understandings of event shower energies.

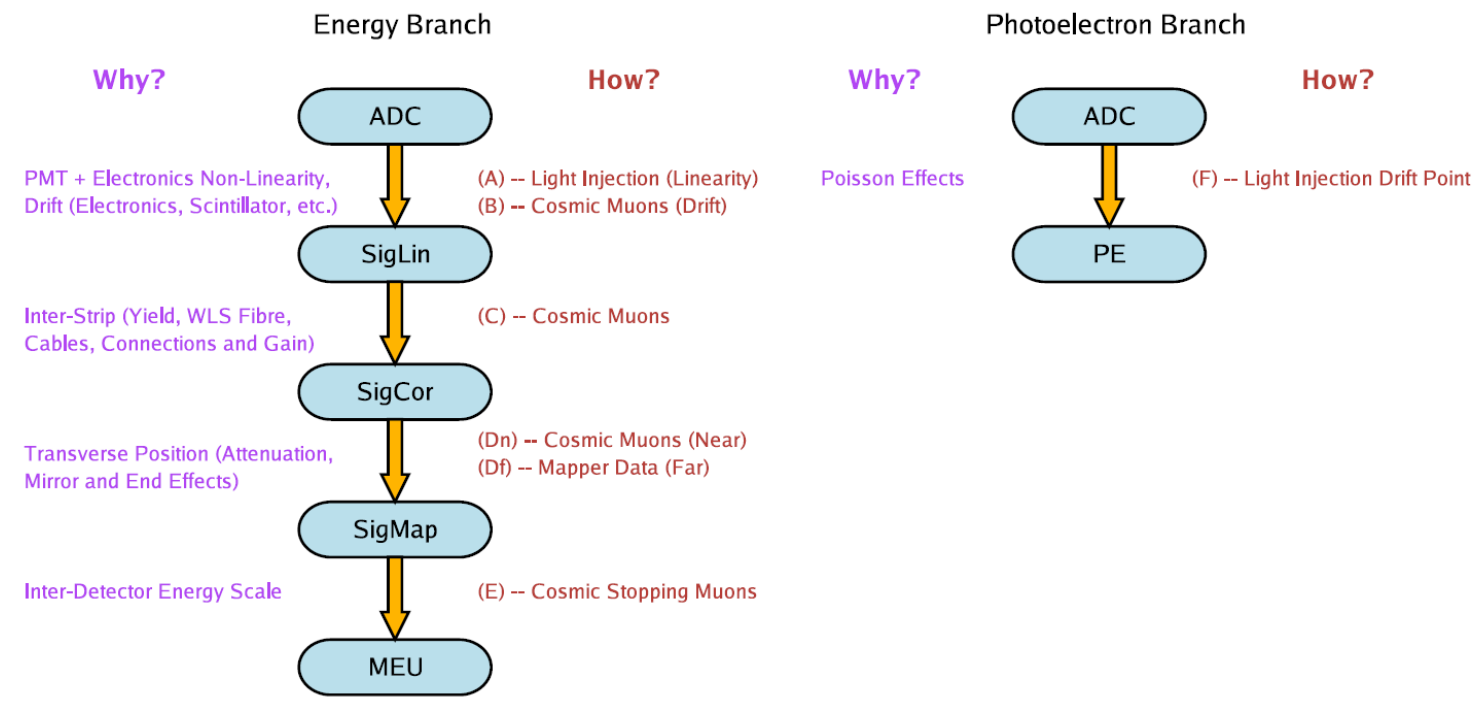

Figure 4.9: Flow diagram of the two calibration chains used by MINOS. Image taken from (28).

\section{Energy Branch}

The Energy Branch takes the raw pulse height in ADCs, defined as $\mathrm{Q}_{\text {raw }}$, and uses a series 
of reweighting corrections to compute $\mathrm{Q}_{\text {corr }}$ - as seen in Expression (4.1), where $i$ is the readout channel, $d$ is the detector, $s$ is the strip, $t$ is the interaction time, and $x$ is the interaction position.

$$
Q_{\text {corr }}=Q_{\text {raw }}(i, d, s, t, x) \times D(d, t) \times L\left(d, s, Q_{\text {raw }}\right) \times S(d, s, t) \times A(d, s, x) \times M(d)
$$

$D(d, t)-$ Drift

The drift correction is implemented to account for changes in the detector response that occur over time. This includes changes that occur in detector elements, such as the scintillator and WLS fibers. These components are susceptible to temperature fluctuations on short time scales, and - over time - the aging of the scintillator and fibers can degrade performance. In addition, the responses of the PMTs and front-end electronics may also change over time. Thus, these systems are monitored, and the recorded data are used in the construction of this correction.

Through-going cosmic ray muons are used to assess the detectors' responses on a day-today basis. The total pulse height per plane of these muon events is measured and the median of the daily distribution is compared experiment's initial median. The ratio of those to quantities is the corrective factor used.

$$
D(d, t)=\text { Initial Median Response }\left(d, t_{0}\right) / \operatorname{Median} \operatorname{Response}(d, t)
$$

As the detectors age and the median response to charged particles diminishes, the drift correction compensates as seen in the from above. The drift in the Far Detector has been roughly $2 \%$ per year over the lifetime of the experiment. The Near Detector, however, has exhibited more dynamic behavior. Following Run I, the drift jumped from nearly 
stable to around $4 \%$ per year. ${ }^{(25)}$

$L\left(d, s, Q_{\text {raw }}\right)-$ Linearity

MINOS has a light injection (LI) system capable of yielding an average of 50 PEs per PMT pixel. This system is used to ascertain the linearity of the PMT response. A 5-10\% nonlinearity is observed in the PMTs and Far Detector electronics when light levels exceed 100 photoelectrons. The LI system pulses at varying light levels and the PMT response is parameterized as a function of the true illumination. The function in the linear response region is extrapolated to the region that exhibits non-linear behavior, and the correction is applied to the ADC count.

$S(d, s, t)$ - Strip-to-Strip

The strip-to-strip correction pertains to differences that arise from hits occurring in different channels. While detector elements were intended to produce uniform responses, different strips, fibers, and electronics can exhibit varying behaviors that need to be considered. Once again, MINOS turns to through-going cosmic-ray muons to assist in the construction of this correction. Energy depositions from these events undergo several corrections that include linearization before the mean strip response is compared to the detector average. For each strip, there exists a correction factor as defined in Expression (4.7.3).

$$
S(d, s, t)=\text { Mean Detector Response }(d, s, t) / \operatorname{Mean} \operatorname{Strip} \operatorname{Response}(d, s, t)
$$

This correction, once applied to the pulse heights, removes fluctuations found to be as large as $30 \%{ }^{(25)}$ 


\section{$A(d, s, x)$ - Attenuation}

Attenuation occurs as light propagates through a WLS fiber. This means that energy depositions which occurred closer to the strip read outs will have larger raw pulse heights compared to an identical event in the middle of the detector. When the detectors were under construction, $5 \mathrm{mCi}$ Cesium-137 $\gamma$ sources were guided along the length of each strip to generate a control signal. Data from this module mapping were fit to the exponential form:

$$
A(d, s, x)=A_{1}(d, s) e^{-x / L_{1}(d, s)}+A_{2}(d, s) e^{-x / L_{2}(d, s)}
$$

where $\mathrm{L}_{1}$ and $\mathrm{L}_{2}$ are two attenuation lengths, and $\mathrm{A}_{1}$ and $\mathrm{A}_{2}$ are normalization constants. Through-going cosmic muons provide a useful crosscheck for this correction factor. A study using both the fit and cosmics at the Near Detector showed an agreement to within $4 \%$; therefore, cosmic muons are used at the Near Detector where there are suitable statistics. At the Far Detector, the Module Mapper fit method is used.

\section{$M(d)$ - Interdetector}

The final step of the Energy Branch calibration chain is accounting for the relative and absolute energy scales of the two detectors. Both of these steps are encompassed in the M(d) factor. While the detectors were designed to be functionally identical, there are still fundamental differences between the two systems. Stopping muons are used to set the relative energy scales in the Near and Far Detectors, something which has not been addressed by any of the previous steps of the calibration chain.

A muon momentum window of 0.5 to $1.1 \mathrm{GeV}$ is established for this step because $\mathrm{dE} / \mathrm{dx}$ varies slowly in this region. The section of a muon track which resides in this domain is 
determined based on a hit's distance from the end of the track. The detector response is then used to set the relative energy scale in Muon Energy Units (MEUs), which has an error of around $2 \% .{ }^{(25)} 1 \mathrm{MEU}$ represents roughly $2 \mathrm{MeV}$ of deposited energy. The relative energy scale is now converted into an absolute energy in $\mathrm{GeV}$ by using the data taken with the CalDet. Stopping muons were used to determine the energy scale of events in the Calibration Detector. The Monte Carlo was then tuned such that simulated muon events in the other detectors had the same energy scale. Once this tuning was performed, the Monte Carlo could be used to simulate the detector response to other events (hadronic showers, electrons, etc...) of specified energies. The detectors' responses to electrons were modeled to better than $2 \%$, while hadronic shower responses were modeled to about $6 \% .{ }^{(29)(30)}$ The energy resolution for hadronic showers was determined to be $56 \% / \sqrt{E} \oplus 2 \%$. The energy resolution for electrons is $21.4 \% / \sqrt{E} \oplus 4 \% / E$, where $\mathrm{E}$ is expressed in $\mathrm{GeV}$ (25) $^{(25)}$

\section{Photoelectron Branch}

The Photoelectron Branch is responsible for converting raw ADC counts into a number of photoelectrons. This operation is performed using the data collected via the LI system. The gain is found for each channel, which is related to the RMS width and mean detector response. The number of photoelectrons can then be determined by dividing the ADC counts in a channel by that channel's gain. The branch is used in reconstruction efforts and in instances where an understanding of the Poisson nature of photon statistics is required. 


\subsection{Monte Carlo}

The appearance analyses rely heavily upon simulations to predict background and signal spectra at the Far Detector. Without simulation, MINOS would be incapable of deducing the parameters associated with neutrino oscillations. The adopted simulation chain addresses three primary areas that will be described in the following subsections.

\subsubsection{NuMI Beam Simulation}

The first piece of the Monte Carlo generation scheme involves the modeling of the NuMI beam. These simulations incorporate hadronic models that address the particle productions associated with proton-target interactions. It also takes into account the propagations of these hadrons through the focusing horns, decay pipe and absorber materials, and models the decays of any unstable particles. Obviously, neutrino production is handled at this stage as well.

FLUKA05 Monte Carlo is used in the target-production stage. Produced particles are then recorded and fed to a GEANT3 rendering of the NuMI beam line, which facilitates propagation modeling. This combination of FLUKA and GEANT is called GNuMI by the collaboration, and it allows us to follow possible decay chains from the target interaction all the way to neutrino production. Decays involving neutrinos are propagated to the Near and Far Detectors, and the kinematics of each event are stored for later use.

\subsubsection{Neutrino Interactions}

Quasi-elastic (QE) and inelastic neutrino scattering interactions are modeled using NEUGEN-V3 (Neutrino Event Generator - Version 3). This program is responsible for handling the many characteristics of a neutrino interaction, ranging from its cross section 
to hadronization.

At this stage of the simulation, various methods for computing the cross section are available to address the different interaction types (NC vs. CC) and energy regimes. The AGKY model is used to handle hadronization processes. Depending on the scale of the hadronic invariant mass, AGKY takes one of three paths. If the hadronic invariant mass is greater than $3 \mathrm{GeV}$, PYTHIA/JETSET is used to model hadronic showers. For invariant masses less than $2.3 \mathrm{GeV}$, a phenomenological model based on KNO scaling is used. In the region between 2.3 and $3 \mathrm{GeV}$, AGKY uses both KNO scaling and PYTHIA/JETSET to model events. It scales the output to ensure the continuity of all simulated variables over the domain of the transition region.

Finally, NEUGEN is once again utilized to simulate the scattering of hadronic shower particles as they exit an iron nucleus. Calculations pertaining to these interactions are handled using the INTRANUKE model, which addresses absorption, charge exchange, and pion scattering.

\subsubsection{Detector Response Modeling}

GEANT3 is used in the MINOS detector simulation to propagate the final states from the steps discussed in Section 4.7.2 and model the subsequent hits in the scintillator material. Information from the NuMI beam simulations is also incorporated, for neutrino interactions used in the detector response model rely upon the stored kinematic data mentioned in Section 4.7.1. Detailed models of the detectors, their halls, and the surrounding rock have been produced for this section of the Monte Carlo generation. A cosmic ray analysis was used to determine the position of the plastic scintillator strips to 
a $1 \mathrm{~mm}$ precision, and the magnetic field strengths of each detector are handled by this simulation.

The PhotonTransport package is then used to simulate scintillation and carry resultant photons to the mock PMT pixel. A Poisson number generator is then used to convert the delivered photons into a photoelectron response. The detector output is then simulated using the aptly-named DetSim package, which takes the PE response and produces an ADC response just like the DAQ chain.

Given the similar topologies of $\mathrm{NC}$ and $v_{\mathrm{e}} \mathrm{CC}$ events, this step of the Monte Carlo production chain is particularly important. Modeling issues, particularly those pertaining to showers, present obstacles that the appearance analyses must overcome in order to successfully separate signal and background. These issues, along with a thorough description of the appearance analyses, will be presented in Chapters 5 and 6. 


\section{Chapter 5}

\section{The Appearance Analyses}

\subsection{Historical Summary}

In the past year, $\theta_{13}$ has progressed from an unknown parameter to one of the most precisely known quantities associated with neutrino oscillations. The $\theta_{13}=0$ hypothesis remained consistent with the observed data until the results of Daya Bay and RENO were released, even though Double Chooz, T2K, and MINOS all presented strong indications that relevant oscillation modes had been detected.

Measuring this mixing angle has long been a goal of the neutrino physics community since the added reaches into leptonic CP-violation and hierarchical studies gained by a definitively nonzero $\theta_{13}$ are appealing. For the majority of its existence, MINOS sought to make the first significant measurement of this parameter using $v_{\mu} \rightarrow v_{\mathrm{e}}$ oscillation events - even though the experiment was designed to ascertain the $v_{\mu}$ survival probability - and many of the tools used in the current analyses were developed in previous generations of the $v_{\mathrm{e}}$ appearance search. ${ }^{(31)}$ The results of the $2011 v_{\mathrm{e}}$ appearance analysis are shown in Figure 5.1. 


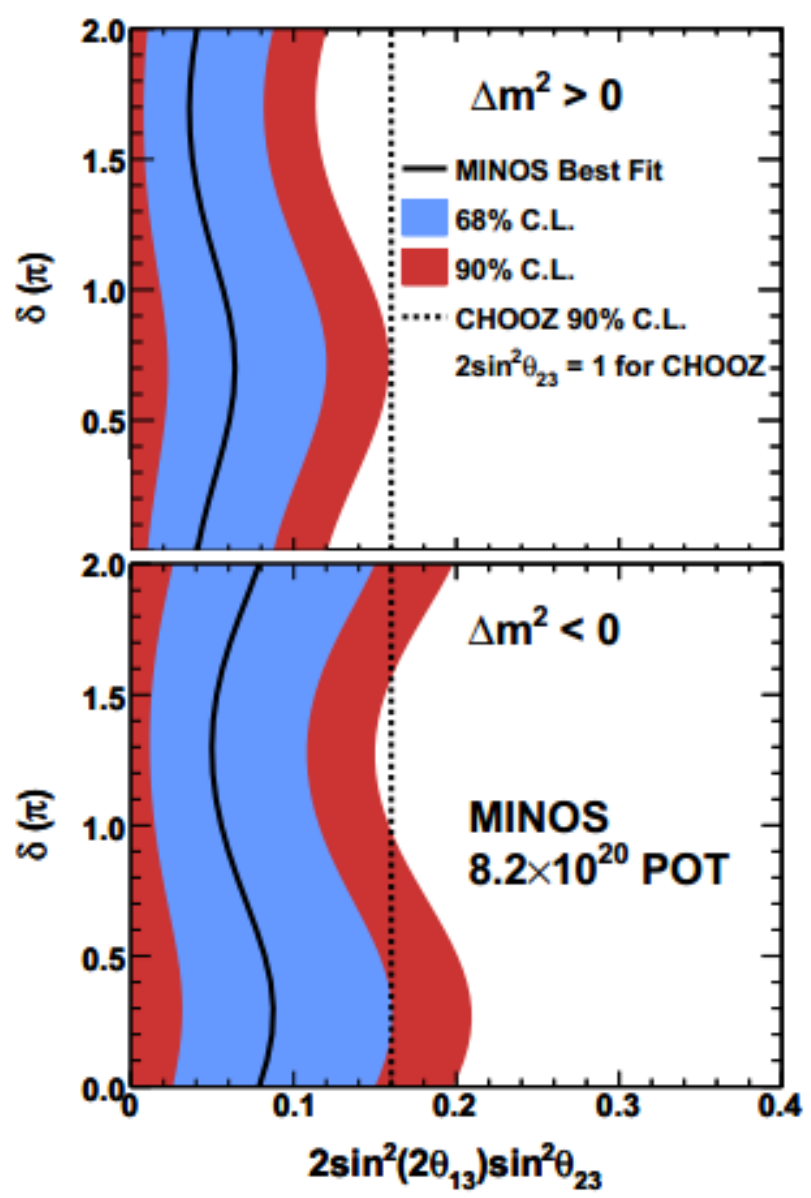

Figure 5.1: Allowed ranges and best fits of $2 \sin ^{2}\left(2 \theta_{13}\right) \sin ^{2} \theta_{23}$ from the 2011 appearance search for both the normal (top) and inverted (bottom) hierarchies. The vertical dashed line marks the CHOOZ $90 \%$ C.L. with a $\theta_{23}=\pi / 4$ and $\left|\Delta m_{32}^{2}\right|=2.32 \times 10^{-3} \mathrm{eV}^{2}$. Image taken from (31).

While the search for the $v_{\mu} \rightarrow v_{\mathrm{e}}$ oscillation mode progressed naturally over the course of the experiment's running, the development of the antineutrino prong of the analysis came as a response to an unexpected result. In 2010, MINOS performed a muon antineutrino disappearance study that demonstrated tension between the new $\bar{\nu}_{\mu}$ contours and the already known $v_{\mu}$ survival parameters. The separation was eventually resolved ${ }^{(32)(33)}$, as shown in Figure 5.2, and the additional antineutrino run periods formed a substantial bulk of the $\bar{v}_{e}$ appearance data used in this thesis. 

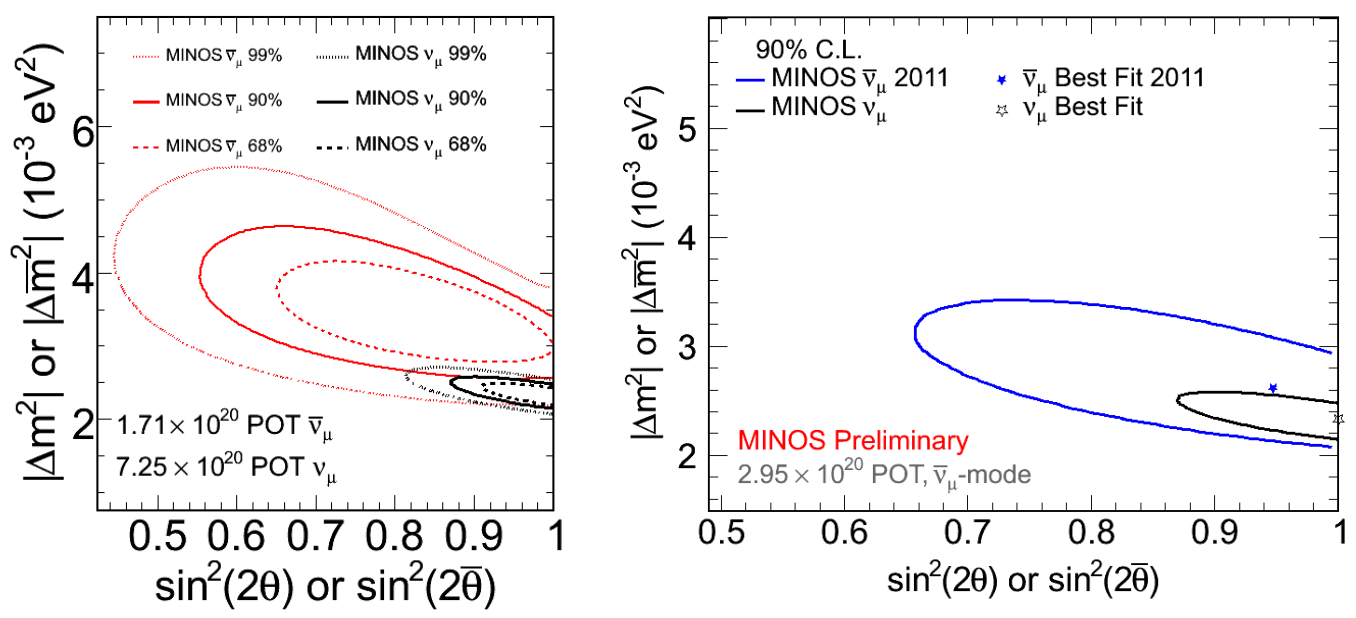

Figure 5.2: MINOS antineutrino disappearance analyses. The left image shows the 2010 result, while the right image displays the result from 2011. The tension between the neutrino and antineutrino mixing parameters was resolved. The appearance group utilized the increased statistics to make a measurement of the parameters associated with $\bar{v}_{e}$ appearance. Figure taken from (32).

The release of the reactor results rapidly changed the scope and direction of the analyses covered by this paper. Prior to the announcement, MINOS was preparing the RHC $\bar{v}_{e}$ appearance analysis for the Neutrino 2012 conference. The discovery of a large (relative to previous estimates) $\theta_{13}$ re-energized efforts to verify the new results with the first joint fit $(\mathrm{FHC}+\mathrm{RHC})$ of the complete MINOS data set. The result also provided the opportunity to use the reactor parameters to glean information about $\delta_{\mathrm{CP}}$ and the mass hierarchy.

\subsection{Analysis Overview}

To measure $\theta_{13}$, MINOS had to search for an excess of $v_{\mu} \rightarrow v_{\mathrm{e}}$ events relative to the predicted background contamination expected at the Far Detector in the no-oscillation hypothesis. The following steps outline the analysis procedure. 


\subsubsection{Data and MC Preparation}

Prior to use, the data and MC sets undergo a series of preparatory calibrations and corrections. This includes the electromagnetic energy calibration, beam and flux corrections, and POT normalization.

The standard MINOS calibration determines shower energy using a hadronic model, but the primary focus of the appearance analyses is electromagnetic (EM) showers.

Therefore, we have to adjust the hadronic energy scale into one suited for EM showers. This conversion is found using simulated quasi-elastic (QE) beam $v_{e} / \bar{v}_{e}$ and signal $v_{e} / \bar{v}_{e}$ CC events. Quasi-elastic events are chosen because the electromagnetic shower typically carries a greater fraction of the event energy compared to non-quasi-elastic (NQE) events.

The deposited energies (in MEU) of QE events are plotted versus the true neutrino energy, and a linear fit is performed over the $1-8 \mathrm{GeV}$ range to ascertain the conversion coefficients.

The coefficients for the $v_{e}$ analysis had been calculated by X. Huang ${ }^{(34)}$ prior to the development of this thesis. The Far Detector scale factor was found to be $23.56 \pm 0.03$ $\mathrm{MEU} / \mathrm{GeV}$, with an offset of $-2.48 \pm 0.09 \mathrm{MEU}$. The Near Detector scale factor was found to be $23.36 \pm 0.18$, with an offset of $-2.76 \pm 0.56 \mathrm{MEU}$.

As an appearance analysis had not been performed on the RHC sample prior to this thesis, the coefficients had to be found using the new antineutrino Monte Carlo. Fitting to the Far Detector $\bar{v}_{e}$ QE profile, the scale factor was found to be $23.46 \pm 0.04 \mathrm{MEU} / \mathrm{GeV}$, with an offset of $-3.49 \pm 0.11 \mathrm{MEU}$. Likewise, the Near Detector $\bar{v}_{e}$ QE profile showed a 
scale factor of $23.28 \pm 0.18 \mathrm{MEU} / \mathrm{GeV}$, with an offset of $-3.93 \pm 0.57 \mathrm{MEU}{ }^{\left({ }^{35}\right)}$

\subsubsection{Signal Selection}

MINOS employs a series of general and "preselection" cuts prior to using the main statistical selection algorithm. The general cuts primarily address issues related to data quality. For example, there are cuts which remove events that occurred during an electronics malfunction or magnetic field downtime. While these cuts are important, the fiducial volume, timing, and cosmic ray cuts are particularly useful.

The fiducial volume cut aims to ensure that an event is fully reconstructed within the detector by removing events with hits too close to the edges of a detector. The fiducial regions for each of the detectors are defined as follows:

\begin{tabular}{|c|c|c|}
\hline Near Detector & $r<0.8$ meters & $1.0<z<4.0$ meters \\
\hline Far Detector & $0.5<r<3.7$ meters & $0.5<z<14.3$ meters \\
& & $16.3<z<28.0$ meters \\
\hline
\end{tabular}

Table 5.1: Fiducial volumes of the MINOS detectors, where $r$ is the radial distance from the center of the neutrino beam, and $z$ is the longitudinal distance with 0.0 meters defined as the upstream edge of each detector. The two $z$ regions associated with the Far Detector indicate the fiducial volumes of each supermodule.

The timing cuts ignore any event that occurred outside a $14 \mu$ s window around a beam spill. This cut aims to minimize the impacts of any background source unrelated to the NuMI beam. In the case when two interactions are seen in one Far Detector spill window, an additional cut is applied which only accepts the event with the highest pulse height. It is assumed that the additional event is an artifact of the reconstruction.

The cosmic ray cut removes events with showers and tracks that have too steep a grade relative to the beam axis to be associated with a signal event. If the angle between a track and the beam axis is greater than $55^{\circ}$ and the vertical projection of the track is longer 
than two meters, the event is excluded. Cosmogenic showers are tagged by finding the pulse-height-weighted average transverse position of activity in each plane. The results for $\mathrm{U}$ and $\mathrm{V}$ planes are plotted versus $z$, and linear fits are applied to determine if the slope of the shower is too steep to be associated with beam-induced activity.

The preselection cuts add additional layers of trimming which remove events whose characteristics strongly resemble or favor beam-related backgrounds. The appearance analyses only use events with reconstructed energies between 1 and $8 \mathrm{GeV}$. While it is possible for signal events to occur outside this range, the above $8 \mathrm{GeV}$ region is dominated by $v_{\mu} \mathrm{CC}$ events and oscillation is improbable. It is more likely to see appearance candidates in the $<1 \mathrm{GeV}$ region; however, the $\mathrm{NC}$ background overwhelms this signal.

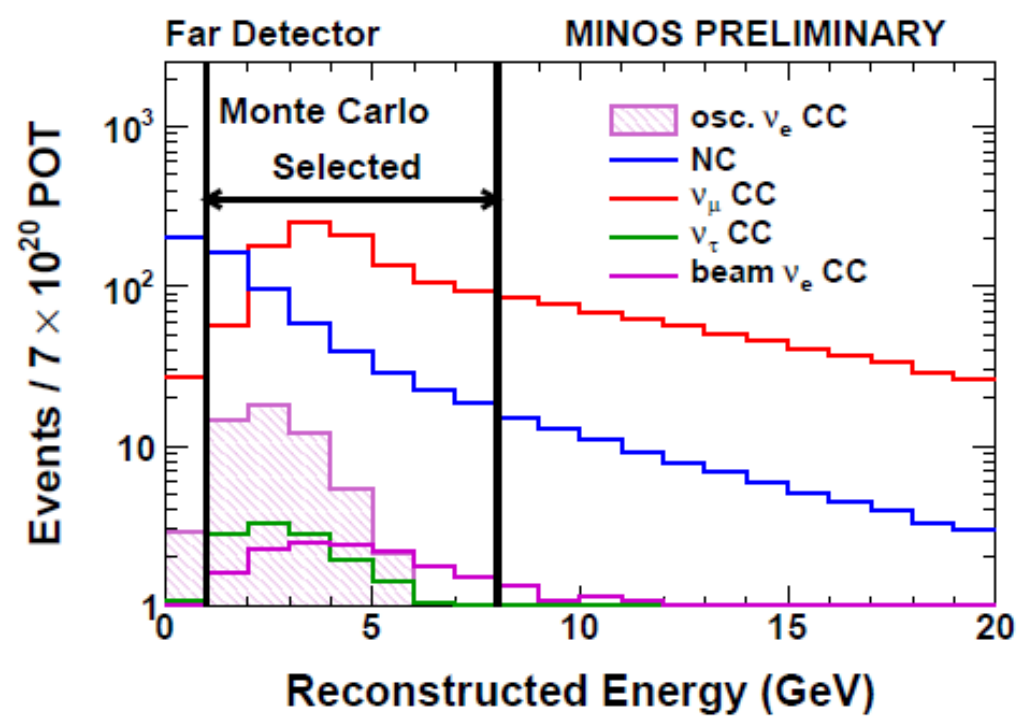

Figure 5.3: Far Detector MC study from the second appearance analysis. The reconstructed energy preselection limits are denoted by the black lines. Image from (36).

To further reject CC backgrounds in the remaining sample, two track based cuts are applied. The first, "track planes", removes any event with a reconstructed track spanning 
more than 24 planes. The second, "track-like planes", eliminates events in which 15 or more planes contain only reconstructed track energy depositions.
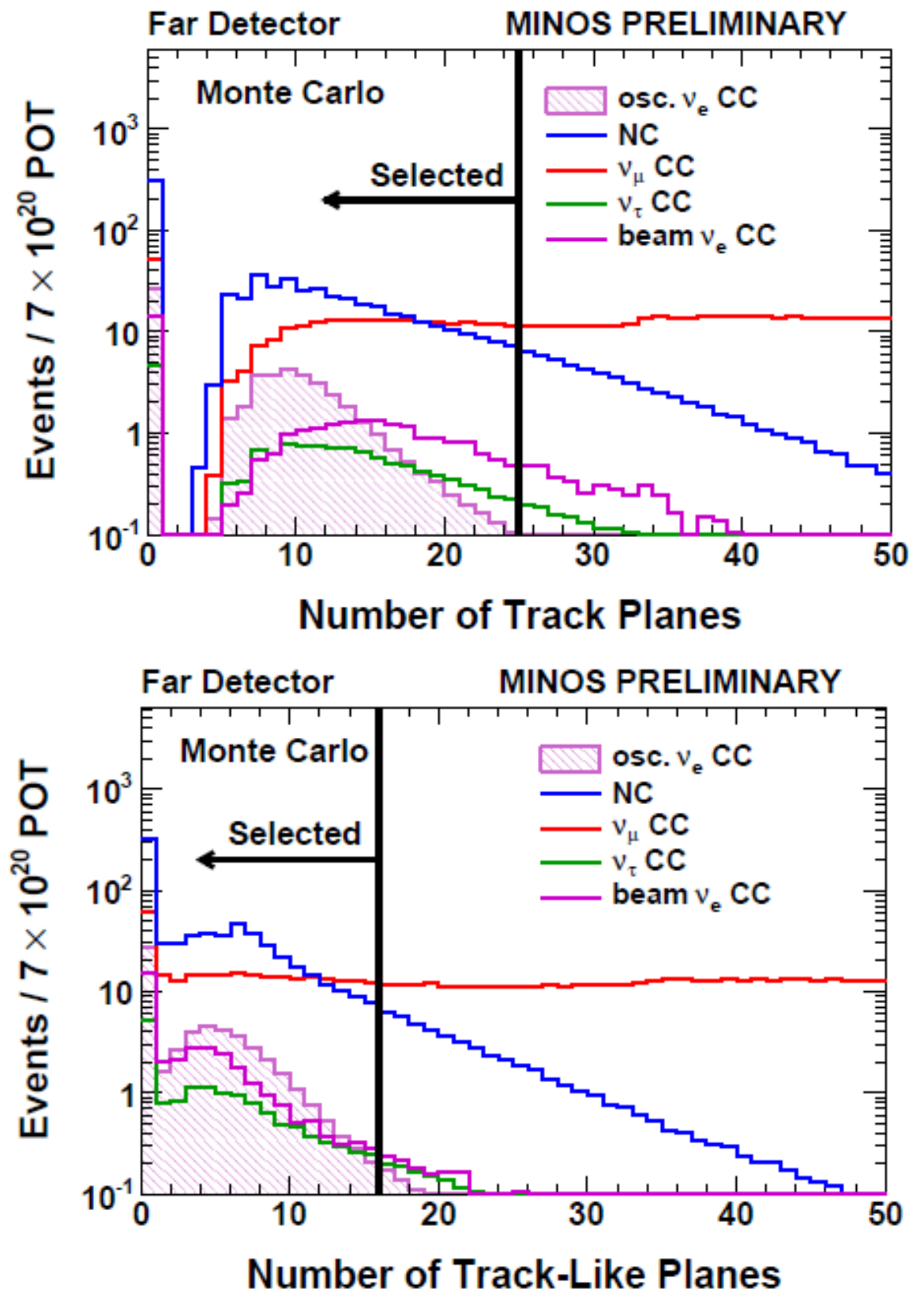

Figure 5.4: Far Detector MC study of track plane and track-like plane cuts from the second analysis. Black bands designate the analysis selected region. Image from (36).

MINOS also requires that events which pass preselection contain at least one 
reconstructed shower as almost all signal events will contain one. To further remove NC backgrounds, we require that at least four contiguous planes receive at least half the energy deposited by a minimum-ionizing particle. This is a useful cut because signal interactions tend to be more compact than hadronic shower counterparts.

The preselection efficiency relative to the fiducial volume cut level was determined for both the FHC and RHC samples using Far Detector Monte Carlo. In the FHC case, $76.8 \%$ of the signal is preserved, while $80.2 \%$ of the background is rejected. In the RHC case, $85.2 \%$ of the signal is preserved, whereas $77.3 \%$ of the background is rejected. To separate the remaining background events from signal candidates, MINOS employs a statistical selection algorithm called LEM (Library Event Matching). This technique was developed primarily for this thesis's predecessor; however, two new algorithms were trained to handle the complete neutrino and antineutrino samples. A single PID discriminant is generated by matching candidate events to a library of Monte Carlo signal and background events. Details of LEM algorithm will be presented in the following chapter.

5.2.3 Near Detector Decomposition - Discrepancies between data and MC modeling prevent us from using only the Near Detector MC to predict the number of expected background events at the Far Detector. To facilitate the decomposition of the Near Detector backgrounds, a data-driven method is used to separate the contributions of each background component. This procedure is called the HOOHE (Horn-On, Horn-Off, High Energy) Method, and it takes advantage of MINOS's variable beam configurations. Each beam configuration contains different fractions of background sources, which 
allows us to construct a set of linear equations to solve for the background components in the standard LE-010cm-185kA running.

$$
\begin{aligned}
& N_{i, T o t a l}^{L E \text { Data }}=N_{i, N C}^{L E \text { Data }}+N_{i, C C}^{L E \text { Data }}+N_{i, \text { Beam }}^{\text {LE Data }} \\
& N_{i, T o t a l}^{H O \text { Data }}=\left[\frac{N_{i, N C}^{H O M C}}{N_{i, N C}^{L E M C}}\right] N_{i, N C}^{L E \text { Data }}+\left[\frac{N_{i, C C}^{\text {HO MC }}}{N_{i, C C}^{L E M C}}\right] N_{i, C C}^{L E \text { Data }}+\left[\frac{N_{i, \text { Beam }}^{\text {HO }} v_{e}}{N_{i, \text { Beam }}^{L E M C} v_{e}}\right] N_{i, \text { Beam }}^{L E \text { Data }} v_{e} \\
& N_{i, \text { Total }}^{H E \text { Data }}=\left[\frac{N_{i, N C}^{H E M C}}{N_{i, N C}^{L E M C}}\right] N_{i, N C}^{L E \text { Data }}+\left[\frac{N_{i, C C}^{H E M C}}{N_{i, C C}^{L E M C}}\right] N_{i, C C}^{L E \text { Data }}+\left[\frac{N_{i, \text { Beam }}^{\text {HE MC }}}{N_{i, \text { Beam }}^{L E M C} v_{e}}\right] N_{i, \text { Beam }}^{L E \text { Data }} v_{e}
\end{aligned}
$$

This system is solved for each analysis bin $i$. Significant Horn-Off and High-Energy statistics have been collected over the course of MINOS's existence, and the bracketed ratios are known from the Monte Carlo. Potentially large errors in the components of the ratio cancel out to first order. The remaining small errors contribute directly to the component predictions of the decomposition.

This scheme yields a set of three equations containing three unknowns, namely the individual background components in the standard LE sample, which can be theoretically determined. Unfortunately, there is an additional complication that needs to be addressed. Both the Horn-Off and High-Energy data sets are dominated by NC interactions, which makes the two equations nearly linearly dependent. Consequently, the uncertainties on the components would be large. To mitigate this problem, the Near Detector Monte Carlo prediction is used to over-constrain the system, and a $\chi^{2}$-minimization fit is performed to determine the decomposition components. This process is detailed in (37). The results of this process for the analyses presented in this thesis will be covered in the results chapter. 5.2.4 Extrapolation - Each background contribution at the Near Detector is extrapolated to the Far using Far/Near Monte Carlo ratios. The mathematical expression for this 
operation is shown below, where $b c$ represents each background contribution and $i$ designates an analysis bin. In this thesis, a 3 PID $\times 5$ Reconstructed Energy binning scheme was used.

$$
\operatorname{Far}_{b c, i}^{\text {Prediction }}=\operatorname{Near}_{b c, i}^{\text {Data }} \times\left(\operatorname{Far}_{b c, i}^{\mathrm{MC}} / \operatorname{Near}_{b c, i}^{\mathrm{MC}}\right)
$$

5.2.5 Systematic Error Analysis - Many of the sources of systematic error cancel out to first order in the Far/Near ratio thanks to the fundamentally identical nature of the MINOS detector designs. However, there are errors which directly skew the Far/Near ratio by predominantly affecting one detector, and there are sources of error that have second-order effects which impact the background prediction. These errors have been identified by previous appearance analyses, and their impacts upon the Far/Near ratio were reanalyzed for the iteration covered by this thesis.

5.2.6 Sideband Checks - Once the prediction has been made and the impact of the systematics has been assessed, we check a set of sidebands prior to looking at the Far Detector data signal region. These checks test the validity of our methods and allow us to address any unforeseen issues without bias. For this thesis, three sidebands were selected. 1. Anti-PID: The Anti-PID sideband contains Far Detector Data outside the signal selected region where no oscillation excess is expected. The Far Detector prediction was compared to the data and a less than $1 \sigma$ difference in the event counts was expected.

2. Glass Box: A large percentage of the FHC dataset used for this thesis was already analyzed in the $3^{\text {rd }}$ appearance analysis. Before unblinding the new FHC data, the new PID algorithm was applied to the old sample to confirm that (to within uncertainties) the same result was found. 
3. MRCC: The MRCC sideband is being retroactively applied post-unblinding. In the past, it has been used to check hadronic shower handling in the signal-selected PID region. As I am leading the work effort to incorporate the MRCC sideband back into the $4^{\text {th }}$ Analysis for our PRD publication, it earned inclusion.

The concept involves removing muon tracks from $\mathrm{CC}$ events to create a signal-free sample of NC-like hadronic showers. As this is, by construction, completely independent of the appearance signals, we are free to investigate the PID region inaccessible to the Anti-PID sideband. A Far Detector prediction is constructed using the MRCC data and $\mathrm{MC}$ events, and a comparison is made to the Far Detector MRCC sample. The historical level of agreement between the prediction and data is $2 \sigma$.

5.2.7 Unblinding - The final step of the analysis chain is actually looking at the events in the signal region. The $90 \%$ confidence intervals of $\theta_{13}$ are calculated over the entire span of $\delta_{\mathrm{CP}}$, and two contours are generated to cover both of the hierarchy possibilities.

\subsection{Analysis Bin Selection Study}

Up until this point, the analysis bins have been mentioned without details being given as to how those bins were selected. To determine the optimal binning scheme, the sensitivities of $2 \sin ^{2} \theta_{23} \sin ^{2} 2 \theta_{13}$ were calculated with various choices of reconstructed energy and PID bin edges.

The information from Near Detector Data and MC and Far Detector MC events is stored in fine-grained energy vs. PID two dimensional histograms. When the events are extrapolated to make the no-oscillation Far Detector prediction, they are rebinned into the various schemes to be tested. It is assumed, by construction, that the number of events 
observed is equivalent to this prediction, and thus the best fit is at $\theta_{13}=0$. In this case, the sensitivity is defined as the upper $90 \%$ confidence level assuming $\delta=0$ and $\theta_{23}=\pi / 4$. The log-likelihood is assessed for incremented $\theta_{13}$ values, which yield an expected excess relative to the best fit, and the point at which $-2 \Delta \operatorname{Ln} L=2.71$ is determined.

In the $3^{\text {rd }}$ appearance analysis, a 3PIDx5E binning scheme was selected with PID bin edges placed at 0.6, 0.7, 0.8 and 1.0; and reconstructed energy bin edges placed at 1,2,3, 4, 5 and $8 \mathrm{GeV}$. For this analysis, the binning selection study was repeated for the RHC sample.

The results of that study indicated that the optimal binning scheme was a 4PIDx4E setup; however, given the small size of the RHC sample, the push to perform the first FHC + RHC joint fit, and the defended use of the $3 \times 5$ scheme, the $3 \times 5$ binning was chosen for the RHC at an accepted loss in sensitivity of 3.1\%. ${ }^{(38)}$

The process defined above can be repeated with different specified values of $\delta_{\mathrm{CP}}$, from which we produce the projected sensitivity contours seen in Figure 5.5. These projections are useful, for a lower $90 \%$ upper limit indicates that the analysis will produce tighter constraints on $\theta_{13}$. 

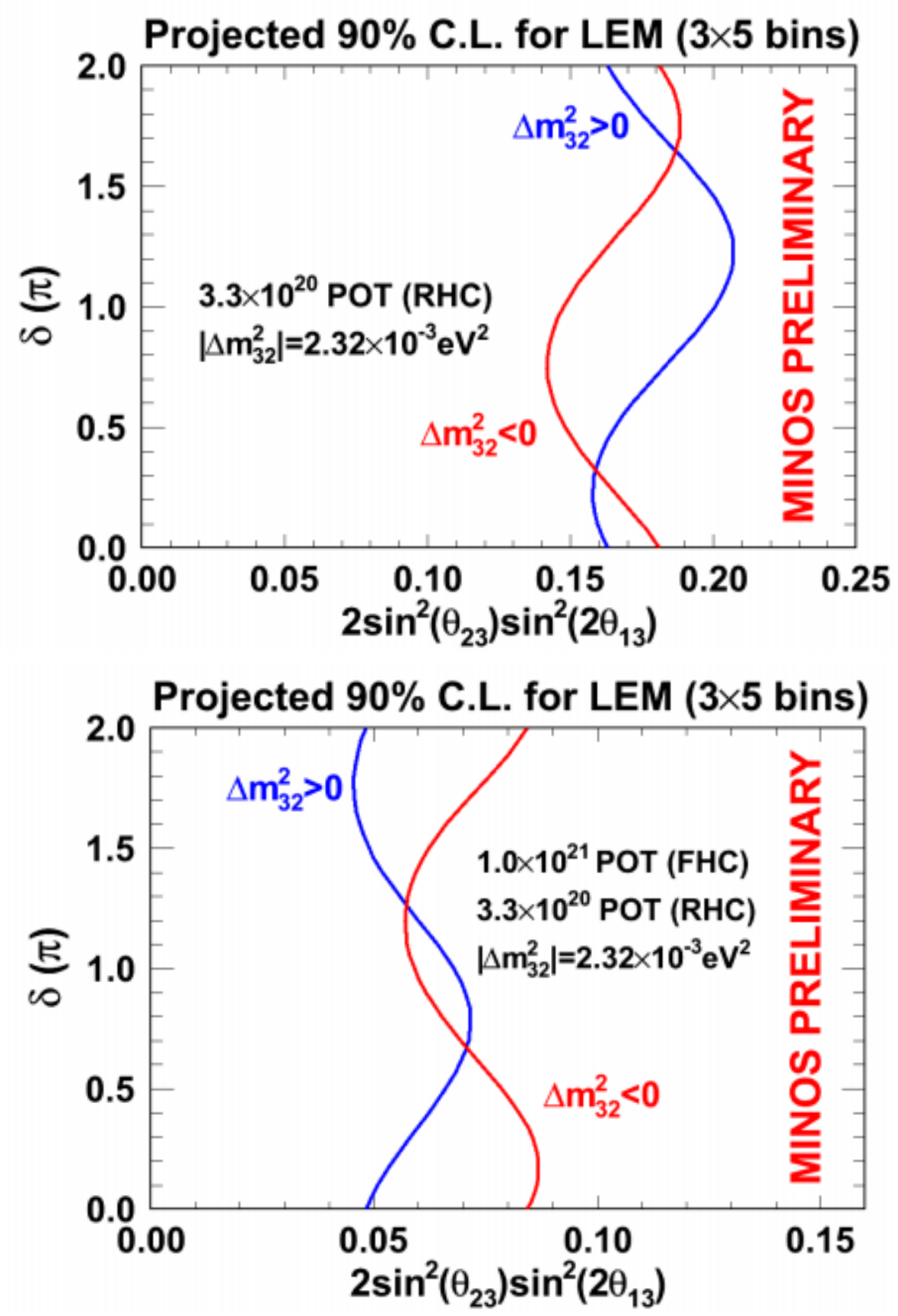

Figure 5.5: Projected sensitivity contours for the RHC sample (top) and the joint sample (bottom). Images taken from (39) and (40), respectively.

The bin selection study was also used to address one of the questions raised in the development of the RHC PID algorithm. Each of the PIDs relies upon a library of MC events to match candidates. The FHC PID, for example, was constructed using a library 
composed entirely of neutrino events. We wanted to determine how transitioning from a neutrino event library to an antineutrino event library would improve the sensitivity of a PID algorithm that had been applied to antineutrino events. Thankfully, during the course of my training, I created a test PID algorithm called $L E M N N B A R$, which categorized RHC events even though it had been trained with the already-existing FHC library. Comparing the LEMNNBAR $3 \times 5$ sensitivity with that of the final RHC algorithm, it was determined that the implementation of the antineutrino library led to a $5.4 \%$ improvement in the RHC sensitivity. ${ }^{(38)}$

\subsection{Data Sets}

The data used for this thesis is divided into runs, typically marked by either the flipping of the horn current or some other change to the NuMI hardware. For the purposes of the joint $\mathrm{FHC}+\mathrm{RHC}$ appearance analysis, the runs have been clustered into three broader periods. Period I contains the FHC dataset which was used in the 3rd appearance analysis (the data associated with the Glass Box sideband). Period II contains the entire antineutrino data, and Period III contains the previously unanalyzed FHC data. Table 5.2 provides the run contents of each of the periods in addition to important demarcating information. Note that the special run configurations are not included in this table. Only the LE- $010 \mathrm{~cm}-185 \mathrm{kA}$ run periods are listed here. 


\begin{tabular}{|c|c|c|c|}
\hline \multicolumn{4}{|l|}{ Period I } \\
\hline Run I & $1.21 \times 10^{20} \mathrm{POT}$ & $2005 / 05-2006 / 02$ & $\begin{array}{c}\text { NuMI Target \#1 (NT-01) } \\
\text { evacuated decay pipe }\end{array}$ \\
\hline Run II & $1.94 \times 10^{20} \mathrm{POT}$ & $2006 / 09-2007 / 07$ & NT-02, evacuated decay pipe \\
\hline Run III & $3.88 \times 10^{20} \mathrm{POT}$ & $2007 / 11-2009 / 06$ & $\begin{array}{c}\text { NT-03, with helium introduced to } \\
\text { the decay pipe }\end{array}$ \\
\hline Run IV(FHC) & $0.08 \times 10^{20} \mathrm{POT}$ & $2009 / 08-2009 / 09$ & $\begin{array}{c}\text { NT-03, short run before first horn } \\
\text { current reversal }\end{array}$ \\
\hline Run V & $0.44 \times 10^{20} \mathrm{POT}$ & $2010 / 03-2010 / 05$ & $\begin{array}{c}\text { NT-03, horn current switched } \\
\text { back to FHC mode }\end{array}$ \\
\hline Run VI & $0.61 \times 10^{20} \mathrm{POT}$ & $2010 / 05-2010 / 07$ & NT-03, with target skewed \\
\hline \multicolumn{4}{|l|}{ Period II } \\
\hline Run IV(RHC) & $1.70 \times 10^{20} \mathrm{POT}$ & $2009 / 09-2010 / 03$ & NT-03, first RHC run period \\
\hline Run VII & $1.23 \times 10^{20} \mathrm{POT}$ & 2010/11-2011/03 & $N T-05$ installed $2010 / 10$ \\
\hline Run IX & $0.41 \times 10^{20} \mathrm{POT}$ & $2011 / 07-2011 / 09$ & $\begin{array}{c}\text { NT-02 Prime, target revived after } \\
\text { multiple target failures }\end{array}$ \\
\hline \multicolumn{4}{|l|}{ Period III } \\
\hline Run VII & $0.12 \times 10^{20} \mathrm{POT}$ & $2011 / 06-2011 / 07$ & $\begin{array}{l}\text { NT-01 Prime, first target revived } \\
\text { after multiple target failures }\end{array}$ \\
\hline Run X & $2.35 \times 10^{20} \mathrm{POT}$ & $2011 / 10-2012 / 04$ & $\begin{array}{l}\text { NT-07 installed } 2011 / 09, \\
\text { return to FHC mode }\end{array}$ \\
\hline
\end{tabular}

Table 5.2: Table displaying the POT exposure, start and end months, and important information for each run. The table is divided into the larger period groupings which were used during the joint analysis. ${ }^{(24)}(41)$ 


\section{Chapter 6}

\section{Library Event Matching (LEM) Particle ID Algorithm}

\subsection{The LEM Concept}

To select $v_{e} \mathrm{CC}$ and $\bar{v}_{e} \mathrm{CC}$ events in the MINOS Far Detector, the LEM PID algorithm is used to distinguish electromagnetic showers from other background interactions. The preselection cuts described in Section 5.2.2 remove a large percentage of the background events from the data, but a statistical selection tool is still needed to identify signal candidates.

The first two appearance analyses, (42) and (36), utilized a PID discriminant produced by an artificial neural network (ANN11) to rank events. While the ANN11 approach brought selection power, it relied entirely upon reconstructed variables. Information is lost during the generation of these derived values, which placed an additional limitation on the sensitivity scope of the ANN11 PID.

The first LEM PID was developed during the $3^{\text {rd }}$ appearance analysis (31) as an attempt to avoid the problems associated with using higher-level variables. This goal is achieved by using the raw strip hits in the detector as the means of discriminating the types of interactions. Candidate events are compared to a library of around 20 million simulated signal, $v_{e} \mathrm{CC}$ or $\bar{v}_{e} \mathrm{CC}$ depending on which sample is being investigated, and about 30 million NC background interactions. Following this comparison process, information from the 50 best matches is fed to an artificial neural network that produces the final PID discriminant.

The LEM approach is a simple concept that yielded the desired improvement in 
sensitivity. In the previous analysis, it was found that the use of the LEM PID produced a $14 \%$ improvement in sensitivity relative to that achieved by the old ANN11 PID.

Unfortunately, the LEM method requires more computer resources than its predecessor.

A great deal of work was made in the previous and current analyses to streamline the matching process. A flowchart summarizing the chain from input event to discriminant is shown in Figure 6.1, and an illustration of the matchmaking process is displayed in Figure 6.2 .

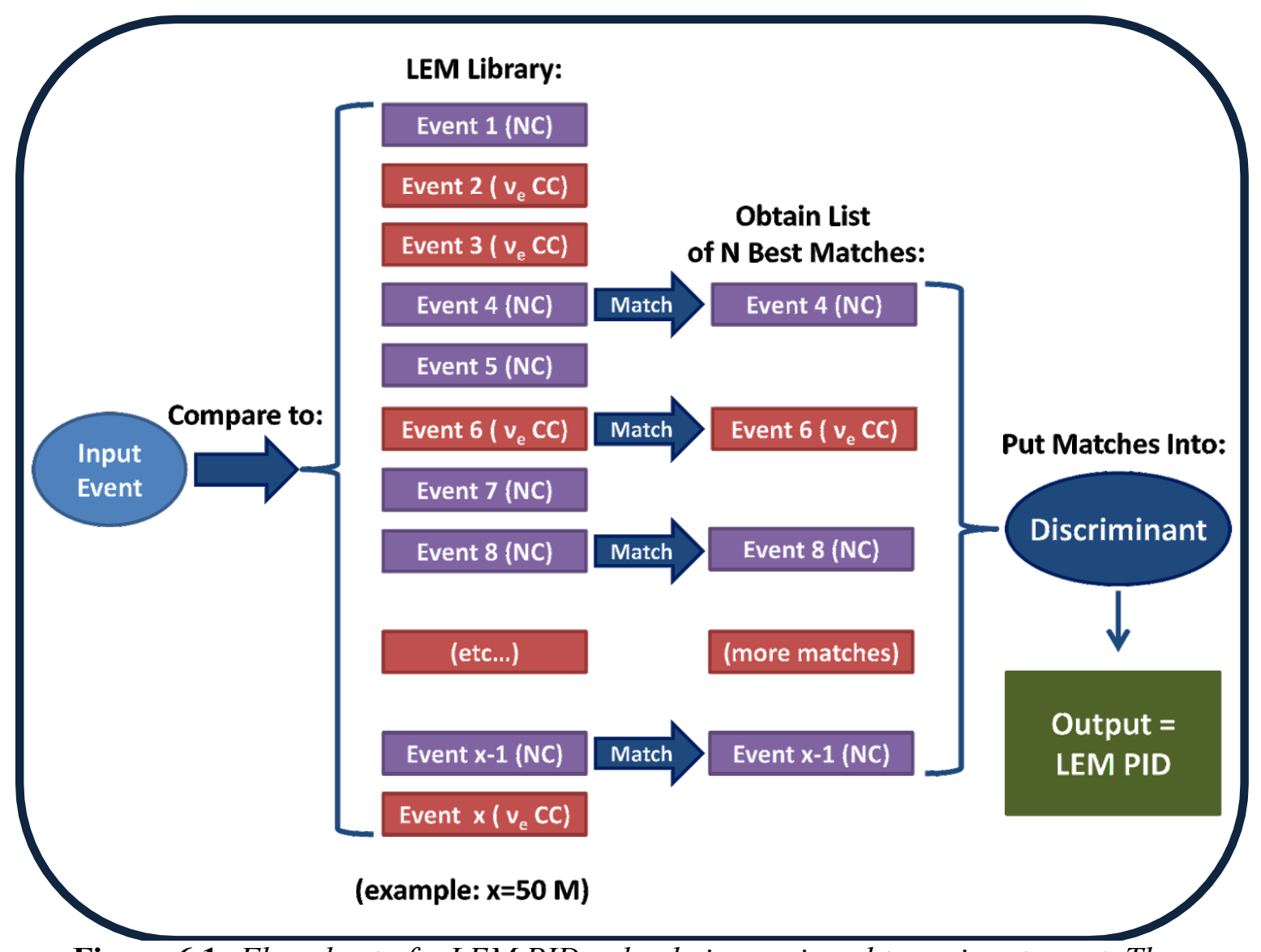

Figure 6.1: Flowchart of a LEM PID value being assigned to an input event. The candidate is matched to various Monte Carlo events in a library. Information from the 50 best matches is used to compute the final output. Image from (27), courtesy of Ruth Toner. 

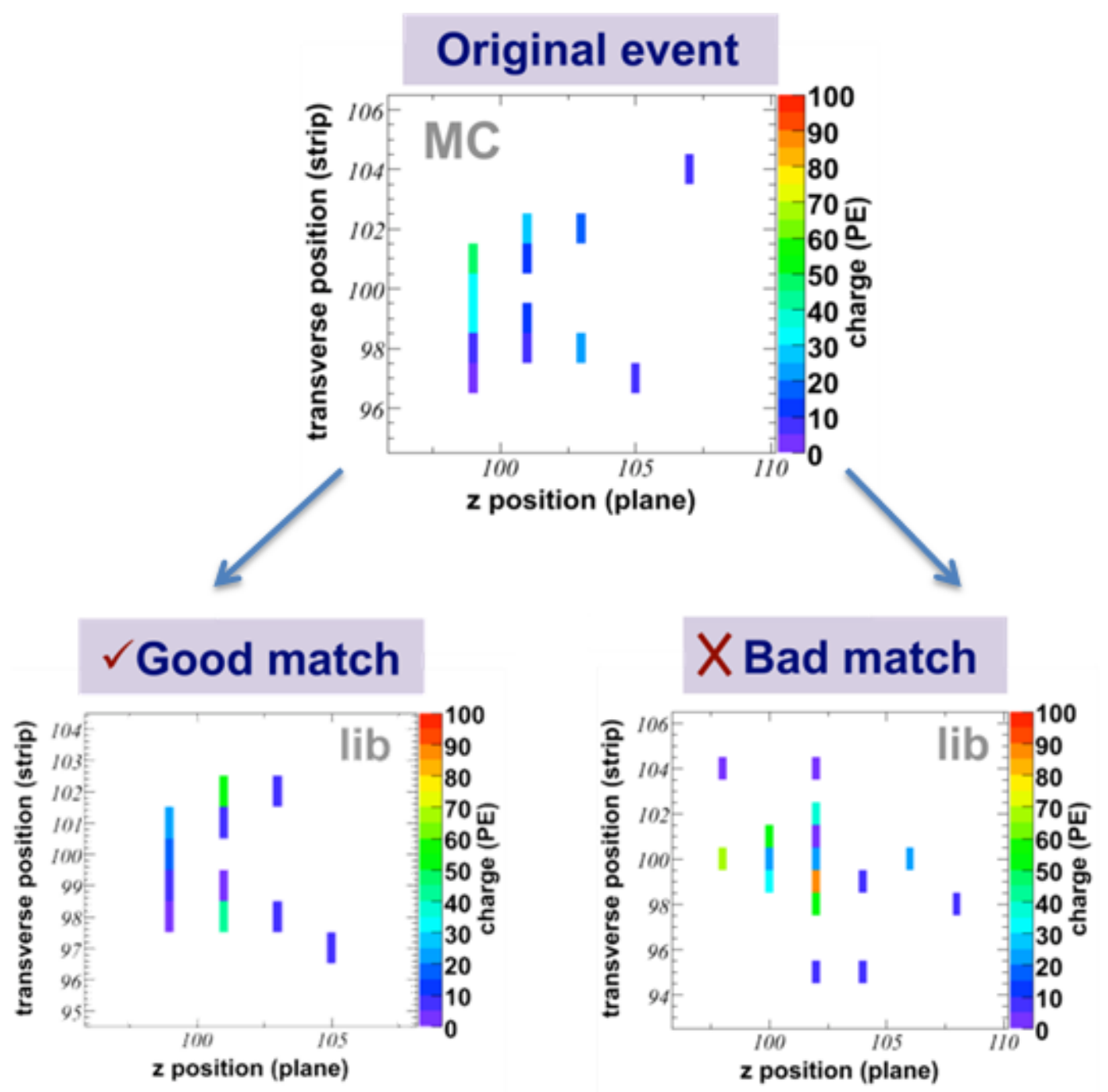

Figure 6.2: Candidate event (top) compared to two library events (bottom). The energy deposition and topology of the bottom-left interaction resembles the candidate event and is thus declared a good match. The event on the bottom-right does not resemble the candidate and is therefore ranked poorly.

\subsection{Thesis Context}

Much of the optimization and implementation work associated with the development of the LEM framework was done in the previous analysis. The efforts of this thesis, with regards to the LEM PID, focused upon the retraining of FHC PID using the latest version of the MINOS reconstruction and the development of the RHC PID utilizing the new 
antineutrino event library. The aim of this work was to produce a pair of fully functional PIDs for both the FHC and RHC event samples that could facilitate the first joint FHC+RHC fit of the appearance data.

\subsection{The Libraries}

The libraries for this thesis were constructed with 50 million neutrino and 50 million antineutrino events. The Monte Carlo was generated using the Fermilab Computing Grid and TACC, and events were reconstructed with the latest analysis-ready package. Each library consists of about $40 \%$ signal events and $60 \%$ NC background events. Muon related events are mostly eliminated by the preselection process described in the previous chapter, and those events that remain in the sample resemble NC interactions. ${ }^{(43)}$ The

optimal library size and composition was determined in a 2008 study by J.P. Ochoa. ${ }^{(44)}$ To decrease processing time, events in the libraries that are not useful to the appearance analyses are screened with an additional set of selection cuts. Events must meet the following criteria to remain in the sample:

1. Events must be contained within the fiducial regions of the detector;

2. $5<\mathrm{N}_{\text {planes }}<30$;

3. $7<\mathrm{N}_{\text {strips }}<80$;

4. $\mathrm{N}_{\mathrm{U} \text {-strips }}>2$;

5. $\mathrm{N}_{\mathrm{V} \text {-strips }}>2$;

6. $50 \mathrm{PE}<\mathrm{Q}_{\mathrm{tot}}<3000 \mathrm{PE}$.

$\mathrm{N}_{\text {strips }}$ is the number of planes traversed by an event. $\mathrm{N}_{\text {strips }}$ equals the number of strips that recorded an event energy deposition. $\mathrm{U}$ and $\mathrm{V}$ specific strip numbers refer to the number 
of strips in that specific plane configuration that received event hits, and $\mathrm{Q}_{\text {tot }}$ is the summed pulse-height, in photoelectrons, of an event. Following this process, approximately 24 million events remain in the library for matching.

\subsection{Match Preparation}

Prior to event matching, additional cuts and changes are applied so that events in the library and signal candidates can be compared on equal footing. All of these procedures were developed in the previous analysis and were reused in this thesis.

\subsubsection{Pulse Height}

Due to large systematic uncertainties in the $\mathrm{ADC} \rightarrow \mathrm{PE}$ conversion, MINOS considered an alternative method for measuring the pulse heights of events during the matching process. As opposed to using the photoelectron branch of the calibration, which introduces large uncertainties through PMT gain corrections, it was decided to use the SigCor units from the energy branch. Events at this point in the calibration chain have been corrected for linearity, drift, and strip-to-strip differences. To convert the deposited charge units from SigCors into units comparable to true photoelectrons, the pulse heights are multiplied by the average photoelectron-SigCor ratio for the relevant detector.

\subsubsection{Charge Attenuation}

After the LEM pulse height correction is made, library events follow the remaining energy branch prescription (with one modification) described in Section 4.7 to account for interdetector effects as well as attenuation. Obviously, events in the detector will have light-levels influenced by the position of interaction in the detector. However, the current libraries are not large enough to properly rank events based upon both their topologies 
and locations in the detector; therefore, care must be taken to ensure that candidate events are not mismatched because of un-calibrated light-levels. To handle this, both LEM candidates and library events undergo an attenuation correction similar to the one in Expression (4.4), shown below.

$$
A(d, s, x)=A_{1}(d, s) e^{-x / L_{1}(d, s)}+A_{2}(d, s) e^{-x / L_{2}(d, s)}
$$

The variables in this function are replaced with constants derived from a phenomenological fit of the data shown in Figure 6.3. This results in the following correction function:

$$
a_{w}\left(d_{w}\right)=\frac{2}{3} e^{-d_{w} / 7.05}+\frac{1}{3} e^{-d_{w} / 1.05}
$$

where $\mathrm{d}_{\mathrm{w}}$ is the distance (in meters) a signal travels along a WLS fiber. A similar correction factor is applied to account for the clear optical fiber between the PMT enclosures and the scintillator modules.

In the Far Detector, pulse heights are read from both ends, making $\mathrm{Q}_{\text {tot }}=\mathrm{Q}_{\mathrm{W}}+\mathrm{Q}_{\mathrm{E}}$, where $\mathrm{E}$ and $\mathrm{W}$ designate the east and west end of the strip. Each contribution to the raw charge is corrected for attenuation, and the sum is reweighted by an overall attenuation factor, $\mathrm{A}^{\mathrm{FD}}$, which ensures that the attenuation-corrected charge levels remain on the same scale of the raw inputs. The same function is used to correct for attenuation in the Near Detector with two modifications: 1) an overall parameter is introduced for interdetector scaling such that the average Near Detector light-level matches that of the Far, and 2) the reflectance of the capped end of Near Detector strips is taken into account. Once this step is completed, events are comparable without worry of position-dependent effects and mismatches. The correction's impact on the observed charge can be seen in Figure 6.4. 
More detailed information regarding the LEM attenuation procedure is documented in (44).

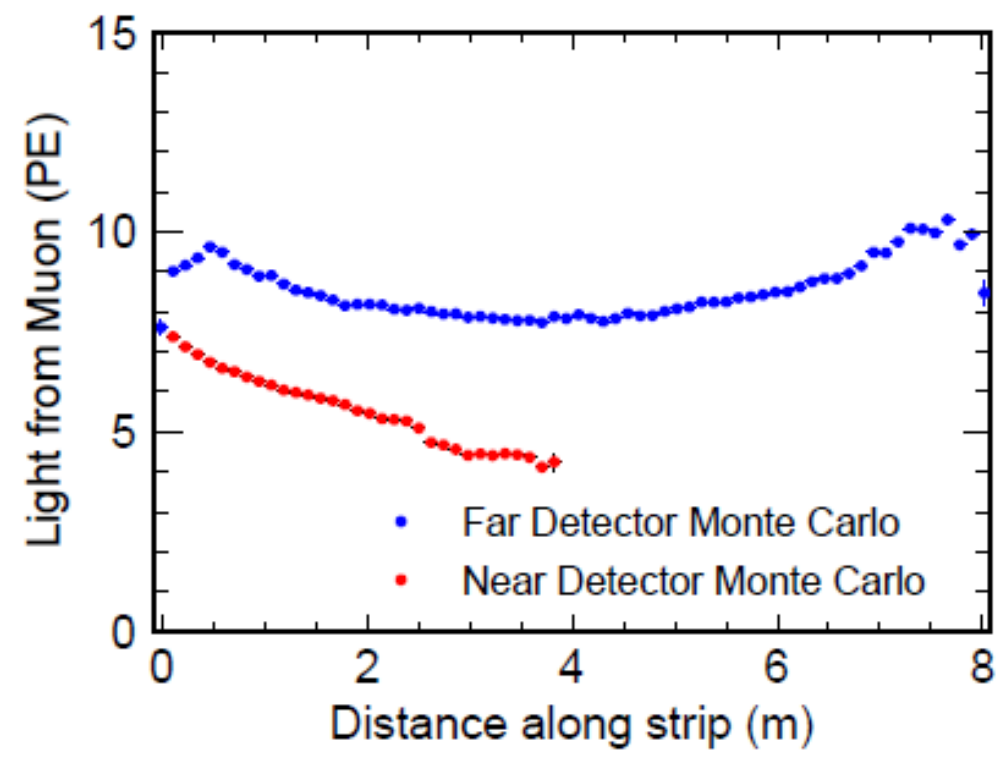

Figure 6.3: Light-levels produced by simulated muon events in the Far (blue) and Near (red) Detectors. A fit to the Far Detector MC produced the function in Expression (6.1), which is used to correct for attenuation in both detectors. Image from (44). 

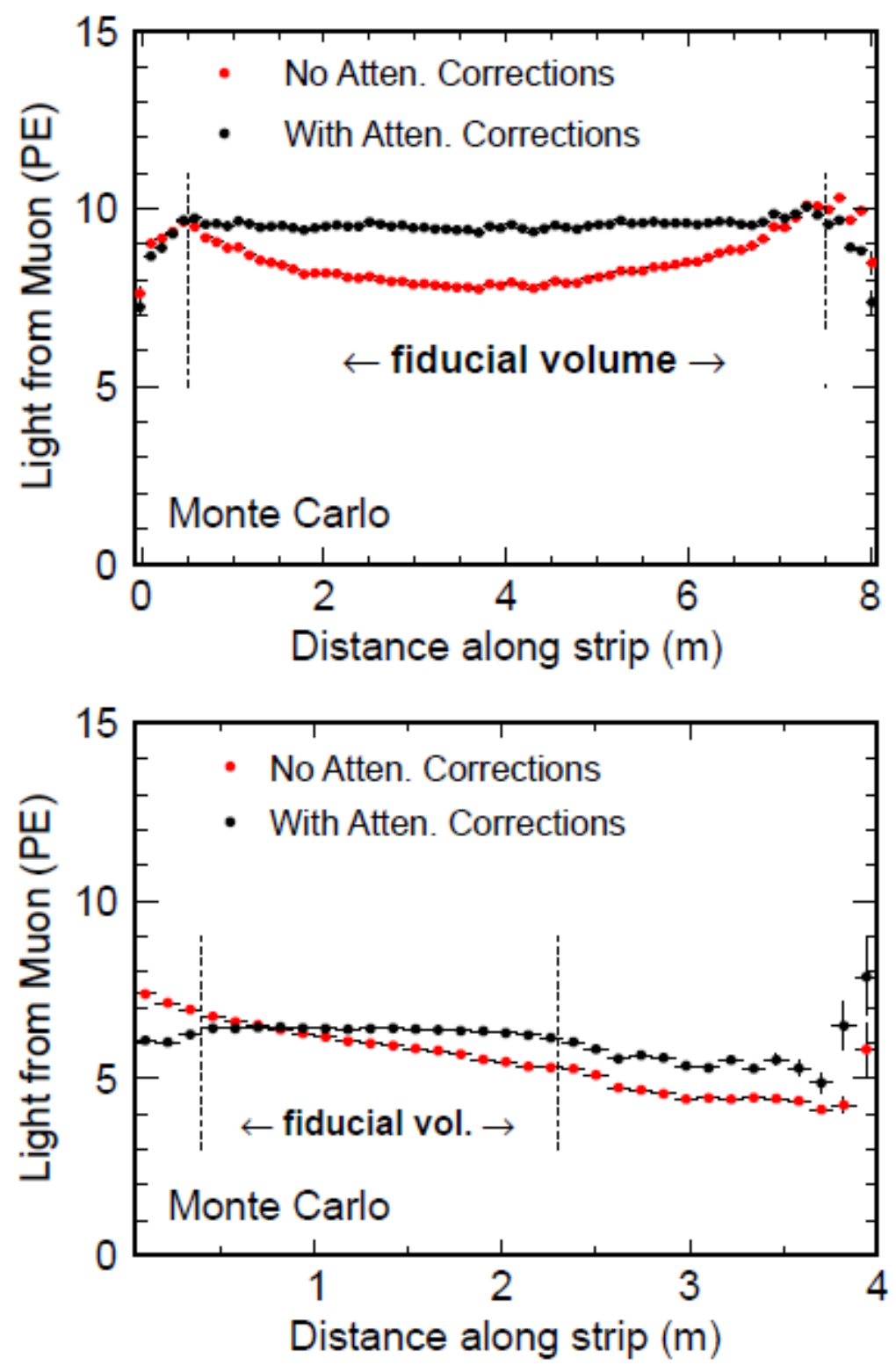

Figure 6.4: Light-levels of simulated muon events the Far (top) and Near (bottom) Detectors. The red lines represent the light-levels prior to the attenuation correction, and the black lines represent the light-levels after the correction has been made. The fiducial region of each detector is indicated by the vertical dashed lines. Note the flatness of the distributions in the fiducial volume. This allows for position-independent matching, which was the goal of the LEM calibration process. Images from (44).

\subsubsection{Event Compacting}

One of the disadvantages of the LEM method is the intensive computing demands needed 
to make, store, and use the library. To reduce the burden on MINOS's resources, as well as streamline the matching process, an event compacting method was developed. The following steps are taken:

1. A 3 PE minimum cut is applied to all strips in the event. This eliminates hits that are likely poorly modeled or the byproducts of PMT crosstalk. A 2 PE cut is already embedded in the reconstruction process; however, the $3 \mathrm{PE}$ cut does a better job reducing the effects of crosstalk-induced interdetector topological variations. ${ }^{(45)}$

2. The pulse-height-weighted mean (centroid) of the event is determined for both $U$ and V planes. Only events in an 8-strip window centered about the strip with the highest summed charge is used to perform this calculation to avoid the inclusion of any unwanted outliers.

3. The attenuation corrections described in the previous section are applied to the event. The position of the centroid is used for the computation of the correction.

4. The event is relocated to a standard position in the detector: plane 100, strip 100. This is done to further the position-independent comparison criterion.

5. Strips are regrouped according to Figure 6.5. This preserves the resolution of hits close to the centroid while de-emphasizing the importance of edge depositions in matching, which favors the compact nature of EM showers over more diffuse NC interactions. Studies done prior to the third analysis determined a $7 \%$ decrease in sensitivity when this compacting method was not utilized. ${ }^{(46)}$ Aside from the sensitivity benefits mentioned in the $5^{\text {th }}$ step of the compacting procedure, this process additionally reduces the file size of library events and candidate inputs. This 
significantly decreases match processing time as well as the burden upon MINOS's limited resources.

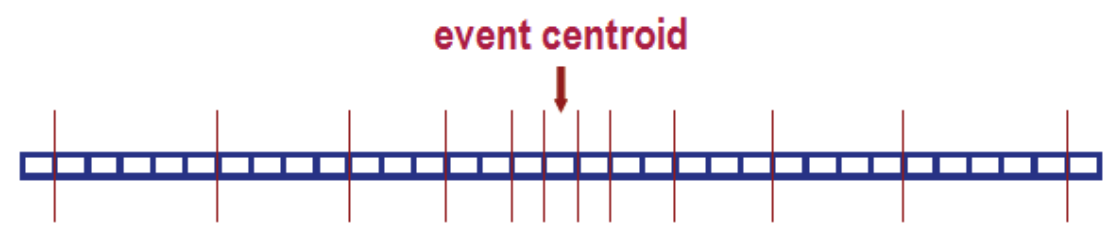

Figure 6.5: This cartoon illustrates the strip grouping outlined in Step 5. Strips (blue boxes) are grouped according the vertical lines. Strips neighboring the centroid (indicated by the arrow) remain unaffected. More strips are compacted into a single group as one moves away from the event centroid, lessening the impact of outlying hits during the matching process.

Figure 6.6 (drawn in the following page, no additional caption included) shows the event displays of a single $4.5 \mathrm{GeV}$ event as it undergoes various stages of the compacting procedure. The raw event displays for both the $\mathrm{U}$ and $\mathrm{V}$ plane views are shown in the top pair of plots. The middle set shows the event after the applications of the PE cut, attenuation corrections, and repositioning. The bottom pair of images show the topology of the event after strip compacting has occurred. 
Raw MINOS Event:
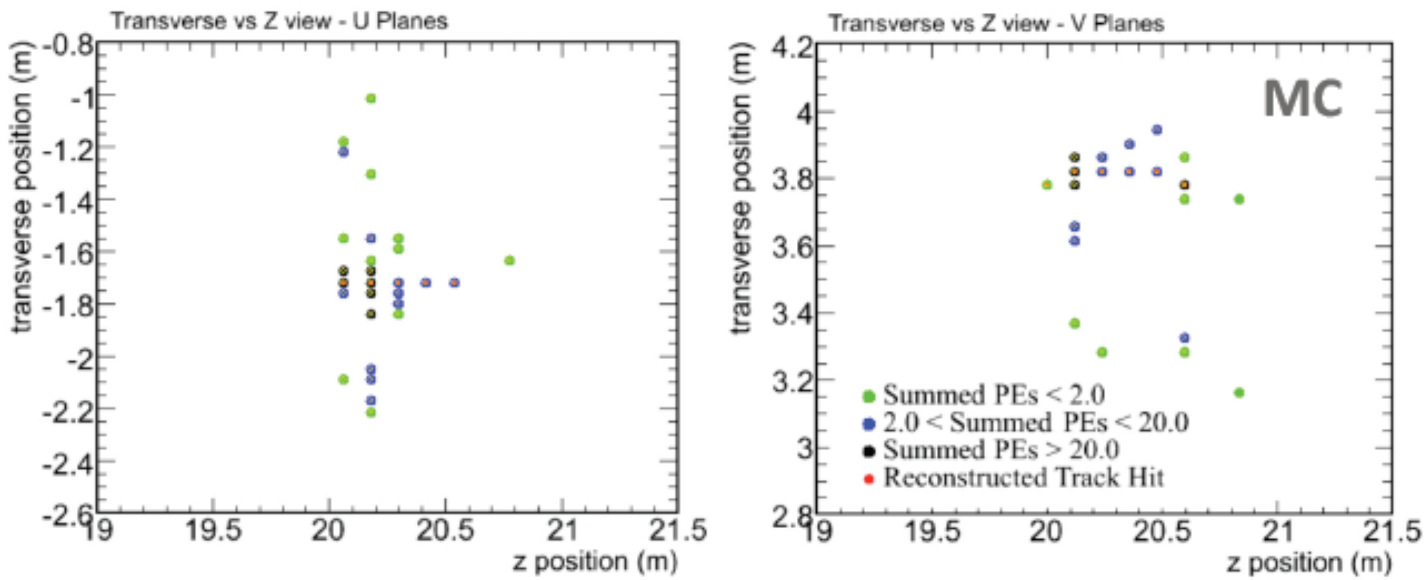

\section{After PE Cut and Repositioning:}
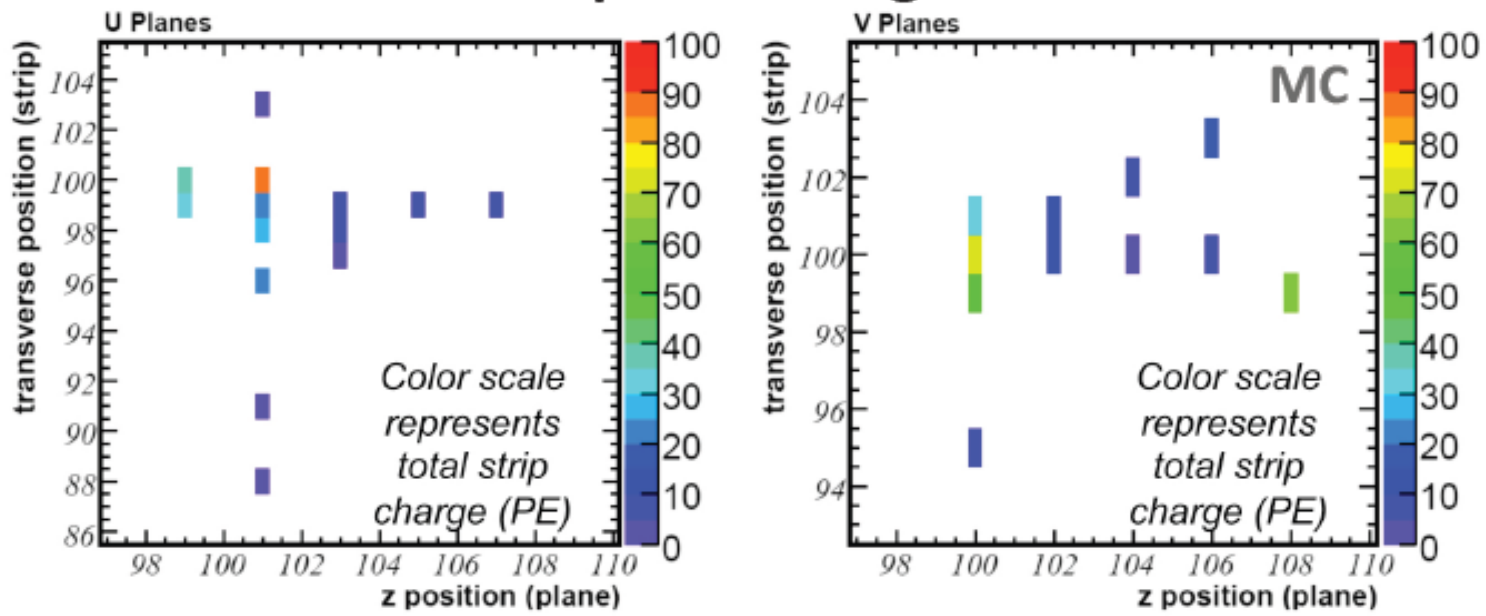

\section{After Strip Grouping:}
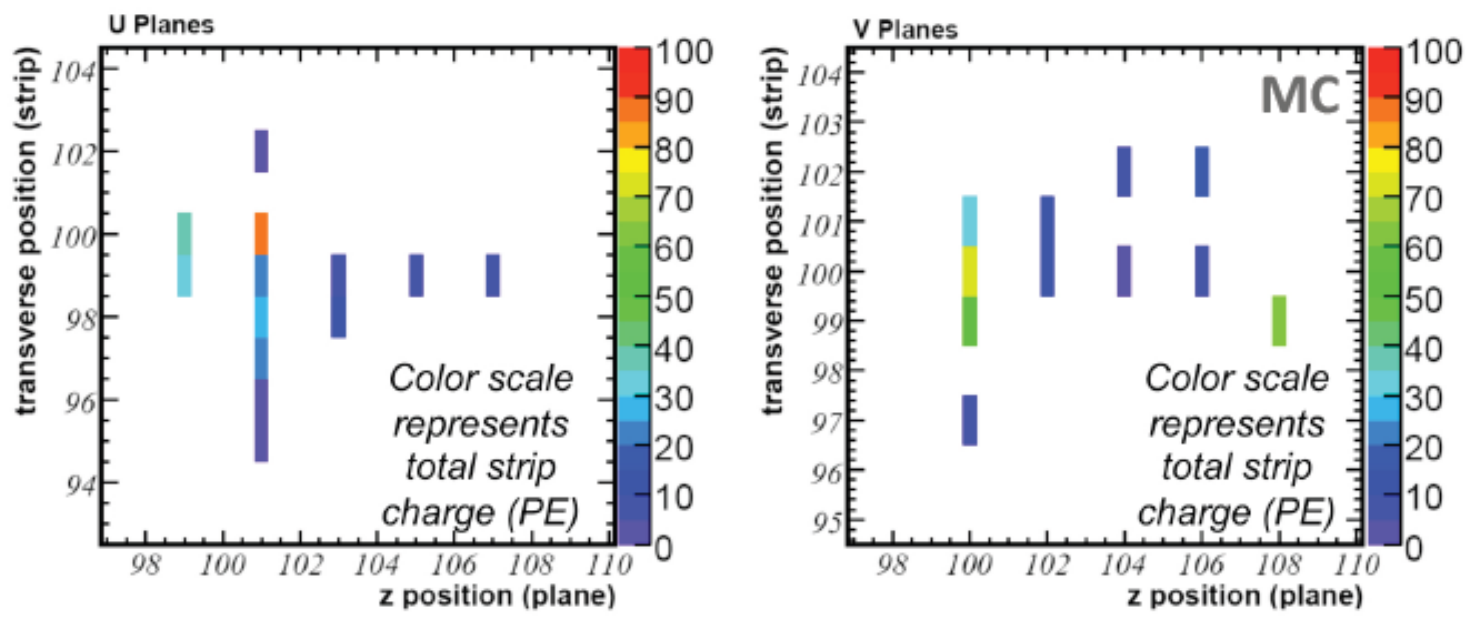


\subsection{Candidate Preselection}

To further reduce the processing time of the matching process, a set of relaxed preselection constraints are applied to the candidate inputs. These cuts select events that occur within the oscillation energy range. In addition, compatibility cuts are applied to ensure that processing time is not being wasted comparing dissimilar events. Any candidate that is completely rejected by the following cuts is assigned a LEM PID value of -1 . The cuts are as follows:

1. $0.5 \mathrm{GeV}<\mathrm{E}_{\text {reco }}<12 \mathrm{GeV}$;

2. $\mathrm{N}_{\text {planes }}<30$;

3. $\left|N_{\text {planes }}^{\text {input }}-N_{\text {planes }}^{\text {lib }}\right| \leq x$, where $\mathrm{x}=4$ if $N_{\text {planes }}^{\text {avg }}=\frac{1}{2}\left(N_{\text {planes }}^{\text {input }}+N_{\text {planes }}^{\text {lib }}+1\right) \geq 20$, $\mathrm{x}=3$ if $20>N_{\text {planes }}^{\text {avg }}>15$, and $\mathrm{x}=2$ otherwise;

4. $2\left|\frac{N_{\text {strips }}^{\text {input }}-N_{\text {strips }}^{\text {lib }}}{N_{\text {strips }}^{\text {ilput }}+N_{\text {strips }}^{\text {lib }}}\right| \leq 0.2$, where $\mathrm{N}_{\text {strips }}$ is the number of compacted strips with hits; 5. $2\left|\frac{\left|\frac{Q_{\text {total }}^{\text {input }}-Q_{\text {total }}^{\text {lib }}}{Q_{\text {total }}^{\text {inpul }}+Q_{\text {total }}^{\text {lib }}}\right|}{\mid}\right| \leq 0.2$, where $\mathrm{Q}$ is the attenuation-corrected charge.

\subsection{Matching Algorithm}

Candidates which pass the aforementioned cuts are matched to events in one of the libraries to facilitate the making of the PID discriminant. The matching procedure provides a quantitative means of determining the likelihood that two different charge topologies were created by the same primary deposition. Consider strip $i$ in the $j^{\text {th }}$ plane of the detector and events $\mathrm{A}$ and $\mathrm{B}$, where the detector's response in event $\mathrm{A}$ was $n_{a}$ photoelectrons and the response in event B was $n_{b}$ photoelectrons. This response is described by Poisson statistics, with the mean photoelectron response defined as $\lambda$. The 
single-strip likelihood is thus written as follows:

$$
l^{j k}\left(n_{a}^{j k}, n_{b}^{j k}\right)=\int_{0}^{\infty} P\left(n_{a}^{j k}, \lambda\right) P\left(n_{b}^{j k}, \lambda\right) d \lambda
$$

where $P(n, \lambda)$ is the probability of observing $n$ photoelectrons given a Poisson-described detector response with mean $\lambda$.

Extending this expression to the full detector response requires taking the product of all $l^{j k}$. This is conveniently expressed in a log-likelihood form shown in Expression (6.3). The summation is over all strips in an event with observed non-zero pulse heights.

$$
-\mathcal{L}=-\sum_{j k} \ln \left(\int_{0}^{\infty} P\left(n_{a}^{j k}, \lambda\right) P\left(n_{b}^{j k}, \lambda\right) d \lambda\right)
$$

The candidate is additionally compared against itself $\left(\mathrm{a}=\mathrm{b}\right.$ in 6.3 ) to determine $-\mathcal{L}_{\text {self }}$, which is subtracted from the candidate-vs.-library likelihood calculations as a constant.

$$
-\Delta \mathcal{L}=\mathcal{L}_{\text {self }}-\mathcal{L}_{\text {comp }}
$$

$-\Delta \mathcal{L}$ is a positive value that gets smaller for better matches between an input candidate and library event.

To reduce the impact of any possible imperfections in the match preparation procedure, $-\Delta \mathcal{L}$ is calculated three times for each candidate-vs.-library comparison. The first calculation is done with the input event's centroid placed at the standard location in the detector (Plane 100, Strip 100). The second and third calculations are done with the centroid's position shifted forward or backward one plane. Of these three computations, the lowest value of $-\Delta \mathcal{L}$ is selected as the true value for the comparison. The initial LEM study determined that approximately one third of input candidates achieved their best matches when the centroid had been shifted. ${ }^{(4)}$ Information from the 50 best matches (events with the 50 lowest values of $-\Delta \mathcal{L}$ ) is used to from the final PID discriminant. 


\subsection{PID Discriminant}

\subsubsection{Post-Matching Variables}

The LEM method has thus far provided a solid foundation for the development of a single-value PID discriminant. In the first and second MINOS appearance analyses, eleven higher-level reconstructed variables were the inputs of the ANN11 artificial neural network, which output a single value used to separate signal events from the collection of candidates. Instead of relying only on the artificial neural network, the LEM method collects information about the candidate by comparing its raw energy depositions to those stored in a Monte Carlo library.

Three variables, constructed using information from the 50 best matches, along with the reconstructed energy of the candidate event are passed as inputs to an artificial neural network that returns a final PID discriminant value. The variable trio was formed to answer fundamental questions pertaining to the appearance analyses:

1. How many of the 50 best matches were $v_{e} \mathrm{CC}\left(\bar{v}_{e} \mathrm{CC}\right)$ events?

2. In those signal $\mathrm{CC}$ events, how dominant was the electromagnetic shower element?

3. How well did the charge distribution of the candidate match the signal CC events?

The first variable, $\mathbf{f}_{\mathbf{5 0}}$, is trivially formed by taking the fraction of the 50 best matches that were signal $\mathrm{CC}$ interactions. This number approaches one if the candidate is more signal like.

The second variable, $\mathbf{y}_{\mathbf{5 0}}$, is composed using the mean inelasticity (hadronic $y$ ) of the signal CC matches. It is defined as follows:

$$
y=\left(E_{v}-E_{l}\right) / E_{v}
$$


where $E_{v}$ is the energy of the incident neutrino and $E_{1}$ is the energy of the outgoing lepton. Signal CC events with dominant electromagnetic showers have y values close to zero, whereas events with more NC-like topologies (e.g. little EM shower activity) have y values closer to 1 . This adds discriminating power to the LEM PID as signal inputs tend to match to low-y library events. In particular, $\mathrm{y}_{50}$ addresses the electromagnetic activity in $\pi^{0}$ decays, which stem from NC interactions, as these events tend to match high-y library Monte Carlo.

The third variable, $\mathbf{q}_{\mathbf{5 0}}$, quantifies the match quality between an input candidate and a library event. Compared to the other variables, $\mathrm{q}_{50}$ necessitates a bit deeper explanation. It is the mean of the matched charge fraction, where $\mathrm{Q}_{\text {frac }}$ is calculated for each signal CC event in the best 50 .

$$
Q_{\text {frac }}=Q_{\text {matched }} /\left(Q_{\text {matched }}+Q_{\text {unmatched }}\right)
$$

The terms in Expression (6.6) are determined in the following manner:

If $n_{a}^{j k} \neq 0$ and $n_{b}^{j k} \neq 0$, (where a and $\mathrm{b}$ are two separate events, $\mathrm{j}$ is the strip index, and $\mathrm{k}$ is the plane index) then $Q_{\text {matched }}=n_{a}^{j k}+n_{b}^{j k}$. If $n_{a}^{j k}=0$ and $n_{b}^{j k} \neq 0$ or if $n_{a}^{j k} \neq 0$ and $n_{b}^{j k}=0$, then $Q_{\text {unmatched }}=n_{a}^{j k}+n_{b}^{j k}$, where one of the $n$ 's is zero.

The mean $\mathrm{Q}_{\text {frac }}$ is then determined and stored as $\mathrm{q}_{50}$ for that input candidate. A larger $\mathrm{q}_{50}$ demonstrates better matching with library signal CC topologies, which indicates that a candidate event is more signal like.

As shown in Figure 5.3 and again in Figure 6.7, $v_{e} \mathrm{CC}$ and $\mathrm{NC}$ events have different reconstructed energy spectra. This stems from the fact that signal CC events are the 
products of oscillation physics which have direct energy dependence. The NC spectrum is unaffected by the oscillation phenomenon, assuming the absence of sterile neutrinos, and thus does not contain the same energy dependence. This justifies the use of a candidate's reconstructed energy as a fourth artificial neural network input.
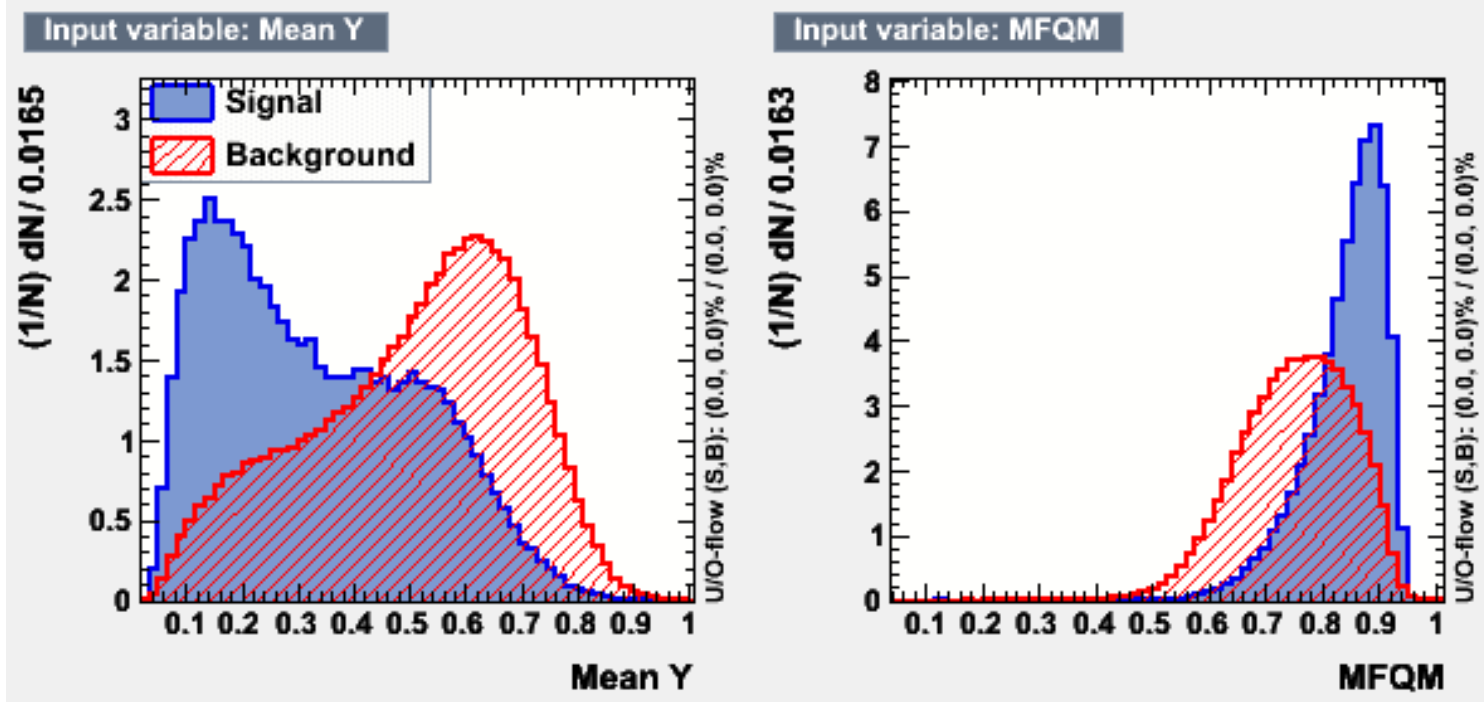

\section{Input variable: FracCC}

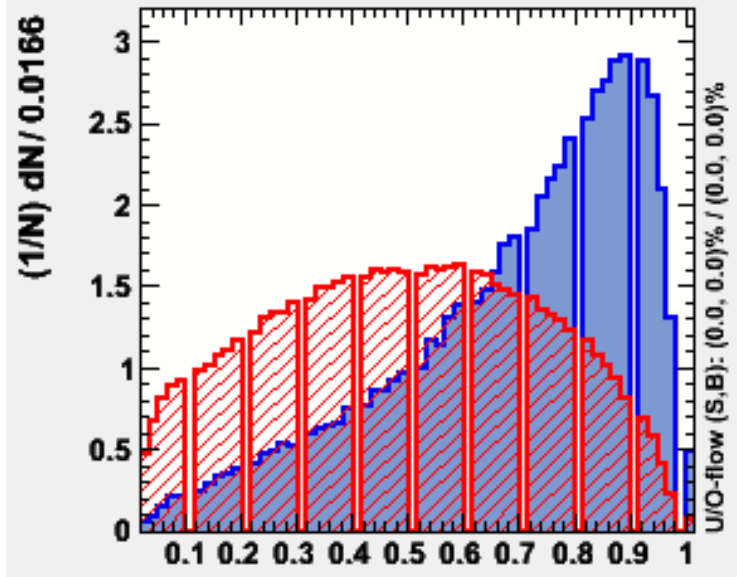

FracCC

\section{Input variable: RecoE}

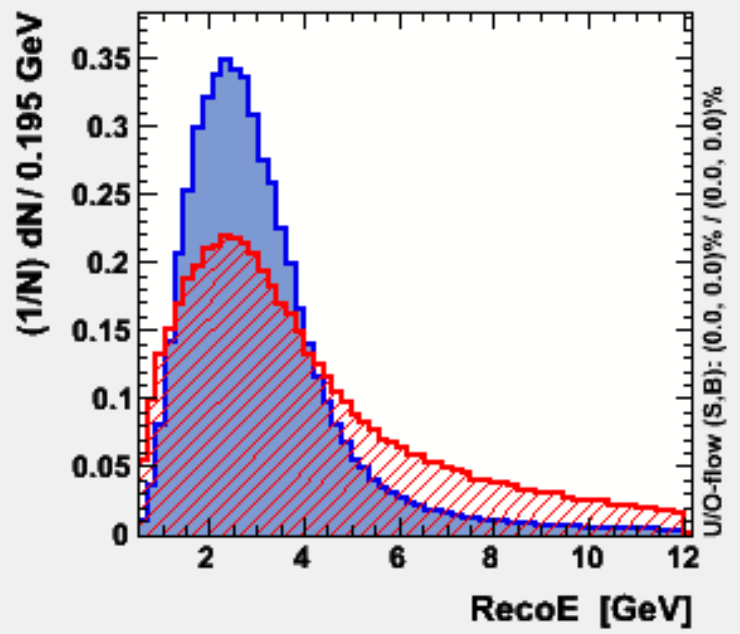

Figure 6.7: Distributions of $y_{50}$ (top-left), $q_{50}$ (top-right), $f_{50}$ (bottom-left), and reconstructed energy (bottom-right) for Far Detector Monte Carlo signal (blue) and background (red) events in the FHC Neural Network training sample. Plots made with the TMVAGui. 


\section{Input variable: Mean $Y$}

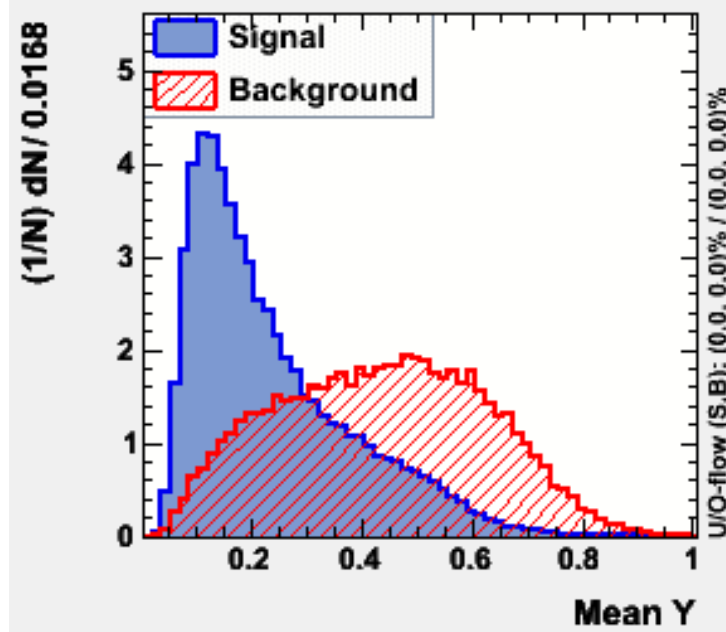

Input variable: FracCC

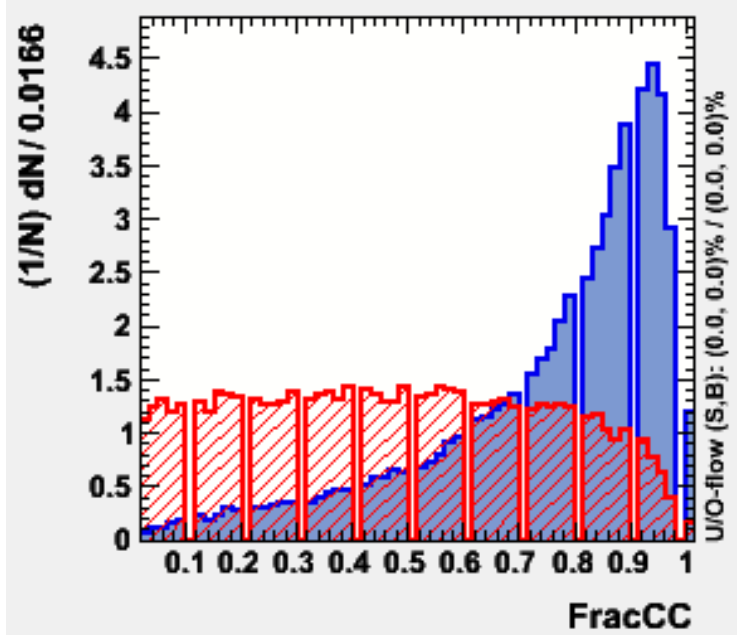

\section{Input variable: MFQM}

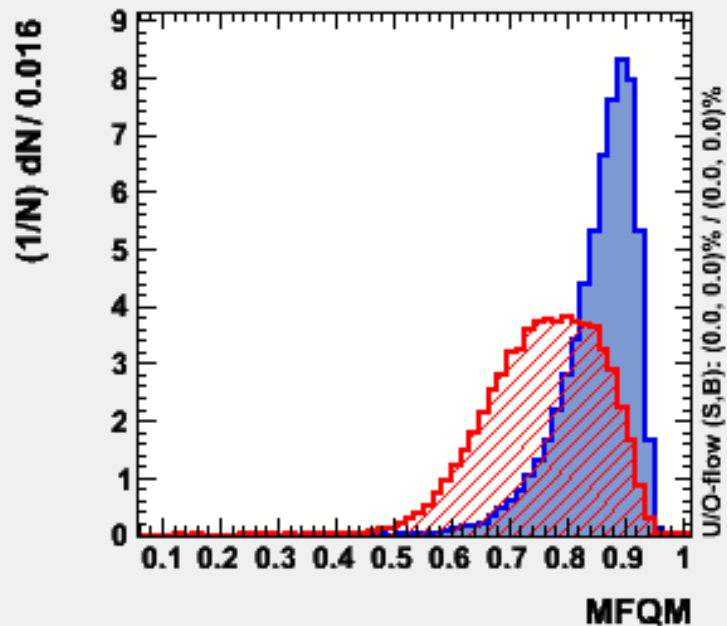

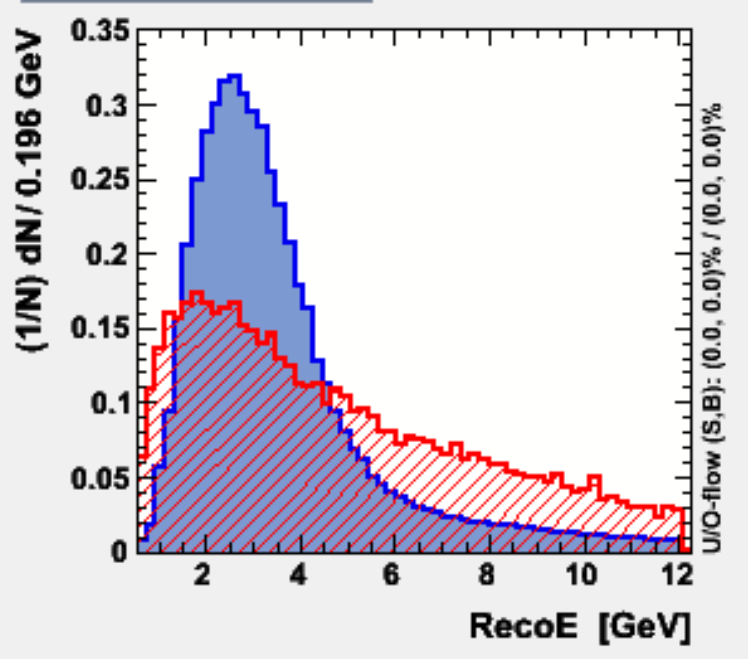

Figure 6.8: Distributions of $y_{50}$ (top-left), $q_{50}$ (top-right), $f_{50}$ (bottom-left), and reconstructed energy (bottom-right) for Far Detector Monte Carlo signal (blue) and background (red) events in the RHC Neural Network training sample. Plots made with the TMVAGui. Note: the gaps in the $f_{50}$ distribution in both this figure and Figure 6.7 are assumed to be due to either sample statistics or a malfunction in the toolkit's plotting function; either way, the gaps have no effect upon the validity of the algorithms.

\subsubsection{The Neural Network}

Two issues motivated the use of an artificial neural network in the appearance analyses.

Naively, one could construct a likelihood function using the probability density functions for each of the variables constructed from the matching process. This approach, however, 
does not account for correlations between the four variables. It does not take much thought to realize that the variables involved are correlated. For example, an event with a dominant EM shower component (small $\mathrm{y}_{50}$ ) will most likely have matched to a large fraction of signal events in the library (high $\mathrm{f}_{50}$ ) and will likely possess a reconstructed energy that agrees with the energy-dependent, oscillation-affected spectrum. A four-dimensional probability density function was considered for the first implementation of the LEM PID to account for the correlations, but such a function could not be sufficiently populated by the statistics available in the Far Detector Monte Carlo. An artificial neural network, however, is perfectly capable of handling the correlations of the input variables as well as the low statistics in the Monte Carlo. It was fully implemented in the $3^{\text {rd }} v_{e}$ appearance analysis and was used to develop the two PID algorithms for this thesis.

The artificial neural network is essentially a set of artificial neurons arranged in a predesignated architecture to mimic the human brain. These neurons receive external inputs and communicate with one another through a network of weighted gates. The system can be trained to perform a particular task, and in this thesis, two neural networks are trained to identify $v_{e} \mathrm{CC}$ or $\bar{v}_{e} \mathrm{CC}$ interactions in our samples. A ROOT package called TMVA (Toolkit for Multivariate Analysis) was used to implement the LEM neural networks. More information about this toolkit can be found in (47).

The setup chosen for the PIDs is referred to as a 4:9:1 architecture. This means that there are three layers of artificial neurons - with each layer containing the labeled number of neurons. The layers function in the following way: 
1. The input layer consists of four neurons, each corresponding to $f_{50}, y_{50}, q_{50}$, and reconstructed energy. These artificial neurons serve as the receptors for the four variables selected from a candidate's matching process.

2. The hidden layer contains nine neurons that separate the input neurons from the output. The weighted connections between these artificial neurons are trained with a process called back-propagation, which adjusts the weights after the responses to known inputs are examined. In this case, the networks were trained using roughly half their respective Far Detector Monte Carlo samples. An output value of 0 is desired for background events, whereas a response of 1 is sought for signal events. After a training cycle, the network outputs are compared against the desired response, and the connection weights are adjusted to minimize the square of the difference. Up to 600 training cycles were performed for each network to match what was done in the $3^{\text {rd }}$ analysis. Once the weights that yield the minimum separation are found, the training process terminates, and the PID is considered ready for use.

3. The final layer contains a single output neuron, which receives a value from 0.0-1.0 that is taken as the final LEM discriminant.

One of the more pertinent issues when using an artificial neural network is verifying that the network behaves properly when given a sample other than its training set. While this is true given any analysis technique, the neural network approach is particularly vulnerable to such criticism because one of the neuron layers is hidden. To validate the training of the PID algorithms, MINOS uses the Far Detector Monte Carlo set aside prior to the training of the network. As the algorithms were not exposed to these samples 
during training, they make perfect independent sets to test the algorithms' validities. The histograms in Figure 6.9 demonstrate separation between the peaks in the background and signal distributions for both FHC and RHC modes when the neural networks process test sample Monte Carlo events.
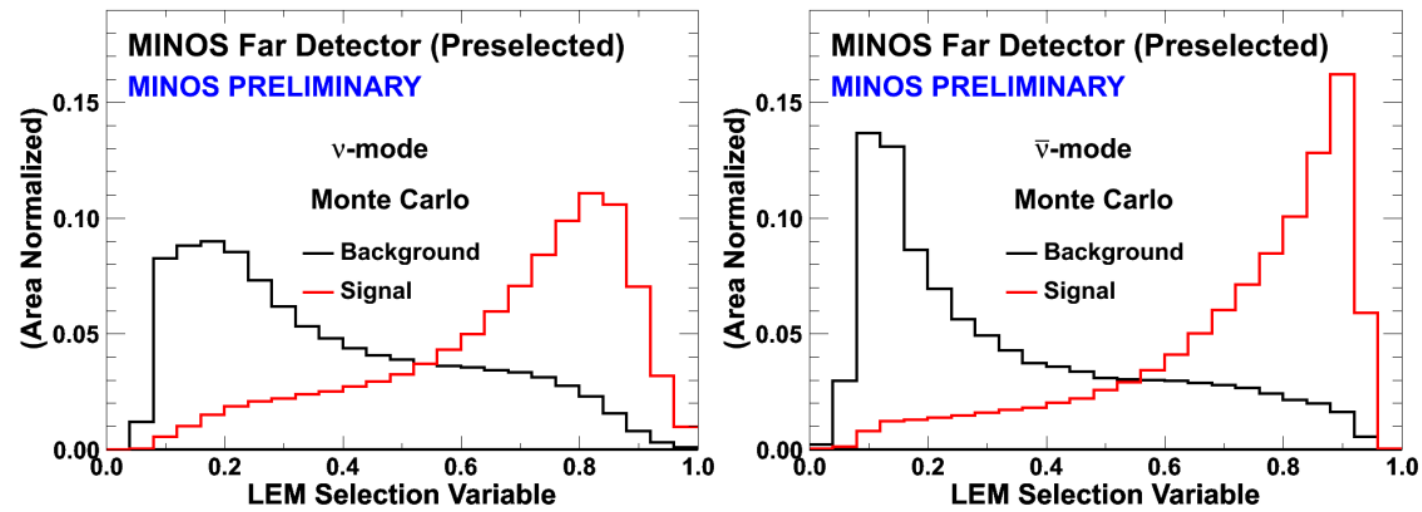

Figure 6.9: Area Normalized PID value distributions of background and signal events in the Far Detector Monte Carlo test sample. The analysis preselection cuts were applied to the sample. The neutrino mode histogram is shown on the left, and the antineutrino mode histogram is shown on the right. Images taken from (27).

Note that the distributions in Figure 6.9 indicate that the RHC LEM algorithm provides stronger separation than its FHC counterpart. The difference is explained by the hadronic $y$ distributions plotted for each sample. Notice in Figure 6.7 that there is significant overlap in the background and signal $y$ distributions for the FHC Monte Carlo. In the RHC sample, the overlap is greatly reduced, for the fraction medium-y events in the signal distribution decrease dramatically. This feature of the distribution can be explained through the nature of the weak nuclear force and helicity arguments.

\subsection{Summary}

The LEM method yields two PID algorithms for this analysis that achieve signalbackground separation in both the FHC and RHC samples by comparing the topology of candidate events to Far Detector Monte Carlo stored in libraries. The technique was 
validated in the previous analysis, ${ }^{(31)}$ largely thanks to the efforts of Ruth Toner and Mhair Orchanian. ${ }^{(48)(24)}$ The work in this thesis extended the implementation of LEM to a larger data set that included both neutrino and antineutrino modes. With an ability to select signal candidates, and the tools in place to make Far Detector predictions, we are capable of analyzing our data. Before this is done, however, sources of error must be addressed and sideband checks must be performed. 


\section{Chapter 7}

\section{Systematic Errors}

As mentioned in Section 5.2.5, many sources of systematic error have minimal impact upon our final results. This is due to the functionally-identical design of the two detectors as well as the fact that our extrapolation relies upon the Far/Near ratio, in which many systematics cancel to first order. Despite this benefit, the appearance analyses are not free from systematic error. Some sources affect one detector more than the other, which biases the Far/Near ratio, whereas others will directly affect the appearance prediction. These sources have been identified and studied in prior analyses, namely the $2011 v_{e}$ appearance search. ${ }^{(31)}$

In the previous study, the effects of nearly 45 systematic errors were determined thanks to available manpower and tremendous processing efforts. For the analyses covered by this thesis, resources had undergone significant reduction. In (48), the impacts of various systematics were ranked, indicating that a large number of errors could be dropped from the analysis without yielding a damaging reduction in sensitivity. The systematic ranking was re-determined using the $3^{\text {rd }}$ analysis extrapolation files that were rescaled to match the POT exposure of the new analysis. ${ }^{(49)}$ Only the systematics selected after this process will be discussed in this chapter.

\subsection{Simulating the Systematic Errors}

Systematic errors were calculated by shifting relevant parameters by $\pm 1 \sigma$. These shifts were achieved either by reweighting associated variables in the Monte Carlo or by producing new reconstructed Monte Carlo with the parameter shift incorporated into the 
process. Regardless of how the parameters in the MC are changed, the end result is the same. We aim to measure the fractional change in the prediction between the shifted and nominal samples. The predictions are combined to form the following expressions:

$$
\begin{gathered}
S_{b k g}^{s t d}=\frac{P_{\mathrm{NC}}^{ \pm 1 \sigma}+P_{v_{\mu} \mathrm{CC}}^{ \pm 1 \sigma}+P_{\mathrm{beam} v_{\mathrm{e}}}^{ \pm 1 \sigma}}{P_{\mathrm{NC}}^{0}+P_{v_{\mu} \mathrm{CC}}^{0}+P_{\text {beam } v_{\mathrm{e}}}^{0}} \\
S_{o s C}^{v_{e, \tau}}=\frac{P_{v_{\mathrm{e}, \tau} \mathrm{CC}}^{ \pm 1 \sigma}}{P_{v_{\mathrm{e}, \tau} \mathrm{CC}}^{0}}
\end{gathered}
$$

In these expressions, $P_{\alpha}^{\beta}$ represents the prediction of component $\alpha$ (e.g. NC) with shift $\beta$. Quantities in the numerator are predictions made with reweighted/modified Monte Carlo, whereas quantities in the denominator are predictions made with nominal/unchanged parameters. The fractional change, $\mathrm{S}$, is used to characterize the systematic errors associated with the appearance analyses.

\subsection{Selected Systematics}

The more serious systematics selected for inclusion in this thesis primarily have direct effects upon the Far/Near ratio used to extrapolate the Far Detector prediction. To study many of these errors, new Monte Carlo reconstruction with pre-shifted quantities is typically required, which threatened to place a large burden on the group's available resources. Thankfully, out of all the systematics which fall into this category, only the absolute and relative energy scale errors lead to a significant degradation of the sensitivity. Errors excluded from this iteration of the appearance analysis changed the sensitivity (relative to the statistics-only value) by $0.06 \%$ on average. Comparing these values to those in Table 7.2, we found it acceptable to make the final prediction using the three energy scale systematics along with those determined via simple reweighting. These 
errors will be discussed in greater detail in the following subsections. It should be noted that, for each analysis, systematic errors were calculated using a covariance matrix that covered the analysis bins discussed in 5.3. In this chapter, reported systematics will represent the error in the Far Detector prediction given a specified range of the LEM PID.

\begin{tabular}{|c|c|}
\hline Systematic & $\begin{array}{c}\text { Change in Sensitivity } \\
\text { (relative to statistics-only value) }\end{array}$ \\
\hline Relative Energy Scale (Near Detector) & $3.13 \%$ \\
\hline Relative Energy Scale (Far Detector) & $0.84 \%$ \\
\hline Absolute Energy Scale & $0.60 \%$ \\
\hline Determined via Reweighting & $3.19 \%$ \\
\hline
\end{tabular}

Table 7.1: The change in sensitivity observed when the energy scale systematics are taken into account. These three errors are determined through using newly reconstructed $M C$. For comparison, the impact of systematics studied using post-reconstruction reweighting is shown in the last row. Information from (49).

\subsubsection{Relative Near and Far Detector Energy Scales}

The relative energy scale systematic represents a large portion of the uncertainty in the final Far Detector Prediction. It arises from several uncertainty sources in the energy calibration chain that includes detector aging, drift, noise, and stopping power. The uncertainty, with regards to the Far Detector prediction, is determined for each detector separately by using the total error on the detector pulse-height-to-MEU scale factor. During the reconstruction process, the energy scale is shifted from its nominal value by size of the factor's uncertainty. These values were reported in (50) to be $1.9 \%$ for the Near Detector and $0.9 \%$ for the Far Detector.

\begin{tabular}{|c|r|r|r|r|r|r|r|r|}
\hline Rel E.S. Det. & \multicolumn{2}{|c|}{$\boldsymbol{v}_{\boldsymbol{e}}$ LEM > 0.6 } & \multicolumn{2}{|c|}{$\boldsymbol{v}_{\boldsymbol{e}}$ LEM > 0.7 } & \multicolumn{2}{|c|}{$\overline{\boldsymbol{v}}_{\boldsymbol{e}}$ LEM > 0.6 } & $\overline{\boldsymbol{v}}_{\boldsymbol{e}}$ LEM > 0.7 \\
\hline $\mathrm{Near}( \pm 1 \sigma)$ & $-2.28 \%$ & $2.46 \%$ & $-2.82 \%$ & $3.64 \%$ & $-2.18 \%$ & $2.56 \%$ & $-2.04 \%$ & $2.23 \%$ \\
\hline $\operatorname{Far}( \pm 1 \sigma)$ & $1.39 \%$ & $-1.33 \%$ & $1.41 \%$ & $-1.26 \%$ & $1.11 \%$ & $-1.46 \%$ & $1.32 \%$ & $-1.50 \%$ \\
\hline
\end{tabular}

Table 7.2: Fractional uncertainties on the standard backgrounds $\left(N C+v_{\mu} / \bar{v}_{\mu} C C+\right.$ beam $v_{e} / \bar{v}_{e}$ ) for the relative energy scale systematic in the specified PID ranges. 


\subsubsection{Absolute Energy Scale}

A $5.7 \%$ error on the absolute energy scale was determined in (50). This error can affect the analysis by propagating through the preselection cuts, which constrain the energy window, and through variations in the neural network inputs, particularly reconstructed energy. Once again, the systematic is studied by introducing newly reconstructed Monte Carlo with the scale factor shifted by a standard deviation.

\begin{tabular}{|c|c|r|r|r|r|r|r|r|}
\hline Abs E.Scale & \multicolumn{2}{|c|}{$\boldsymbol{v}_{\boldsymbol{e}}$ LEM $>\mathbf{0 . 6}$} & \multicolumn{2}{|c|}{$\boldsymbol{v}_{\boldsymbol{e}}$ LEM $>\mathbf{0 . 7}$} & \multicolumn{2}{|c|}{$\overline{\boldsymbol{v}}_{\boldsymbol{e}}$ LEM $>\mathbf{0 . 6}$} & \multicolumn{2}{|c|}{$\overline{\boldsymbol{v}}_{\boldsymbol{e}}$ LEM $>\mathbf{0 . 7}$} \\
\hline std bg. $( \pm 1 \sigma)$ & $0.41 \%$ & $-0.09 \%$ & $-0.18 \%$ & $-0.53 \%$ & -0.95 & $0.97 \%$ & $0.58 \%$ & $0.72 \%$ \\
\hline$\nu_{\tau} \mathrm{CC}( \pm 1 \sigma)$ & $6.50 \%$ & $-5.85 \%$ & $9.66 \%$ & $-9.11 \%$ & $7.34 \%$ & $-7.97 \%$ & $8.67 \%$ & $-8.63 \%$ \\
\hline Signal $( \pm 1 \sigma)$ & $2.20 \%$ & $-2.68 \%$ & $4.21 \%$ & $-5.07 \%$ & $2.20 \%$ & $-2.58 \%$ & $3.24 \%$ & $-3.70 \%$ \\
\hline
\end{tabular}

Table 7.3: Fractional uncertainty in the prediction of the std. backgrounds, $v_{\tau} C C$, and signal events observed when the absolute energy scale is shifted by a standard deviation.

The uncertainty leads to relatively large errors, particularly in the $v_{\tau} C C$ prediction.

\subsubsection{EM vs. Hadronic Energy Scale}

Information from the Calibration Detector indicates that there is a $5 \%$ uncertainty on the relative scaling between electromagnetic and hadronic energy scales. These analyses seek to separate signal and background events based upon the topological differences between EM and hadronic showers. In addition, one of the neural network inputs directly uses the fraction of the EM shower energy relative to the rest of the event. The systematic effect arises from the fact that the energy scales of both components may vary with respect to one another in a completely uncorrelated manner. This uncertainty is studied by reweighting the hadronic energy of an event by $\pm 5 \%$ and by shifting the total reconstructed energy by $5 \%$. The reweighing determines the uncertainty's effect on the selection efficiency, while the energy shifting determines its effect on the reconstructed energy. 


\begin{tabular}{|c|r|r|r|r|r|r|r|r|}
\hline EM vs Had. & \multicolumn{2}{|c|}{$\boldsymbol{v}_{\boldsymbol{e}}$ LEM $>\mathbf{0 . 6}$} & \multicolumn{2}{|c|}{$\boldsymbol{v}_{\boldsymbol{e}}$ LEM $>\mathbf{0 . 7}$} & \multicolumn{2}{|c|}{$\overline{\boldsymbol{v}}_{\boldsymbol{e}}$ LEM $>\mathbf{0 . 6}$} & \multicolumn{2}{|c|}{$\overline{\boldsymbol{v}}_{\boldsymbol{e}}$ LEM > 0.7 } \\
\hline std bg. $( \pm 1 \sigma)$ & $0.27 \%$ & $-0.32 \%$ & $0.18 \%$ & $-0.23 \%$ & $1.04 \%$ & $-0.23 \%$ & $1.53 \%$ & $-0.28 \%$ \\
\hline$v_{\tau} \mathrm{CC}( \pm 1 \sigma)$ & $-0.45 \%$ & $0.19 \%$ & $-0.90 \%$ & $0.74 \%$ & $-0.86 \%$ & $0.88 \%$ & $-1.32 \%$ & $-1.37 \%$ \\
\hline Signal $( \pm 1 \sigma)$ & $-0.09 \%$ & $-0.14 \%$ & $-0.56 \%$ & $0.44 \%$ & $0.04 \%$ & $0.05 \%$ & $-0.42 \%$ & $0.52 \%$ \\
\hline
\end{tabular}

Table 7.4: Fractional uncertainty in the prediction of standard backgrounds, $v_{\tau} C C$, and signal events due to the EMvsHad energy scale uncertainty.

\subsubsection{Normalization}

There are several sources of uncertainty that affect the overall normalization of the prediction. These sources include the fiducial masses, the relative live time, and the relative POT exposure of the two detectors. The uncertainty's contribution to the Signal and $v_{\tau} \mathrm{CC}$ prediction arises from selection efficiency differences between the two detectors. The error is constructed by adding the above uncertainties in quadrature.

\begin{tabular}{|c|c|c|c|c|c|c|c|c|}
\hline Norm. & \multicolumn{2}{|c|}{$\boldsymbol{v}_{\boldsymbol{e}}$ LEM $>\mathbf{0 . 6}$} & \multicolumn{2}{|c|}{$\boldsymbol{v}_{\boldsymbol{e}}$ LEM $>\mathbf{0 . 7}$} & \multicolumn{2}{|c|}{$\overline{\boldsymbol{v}}_{\boldsymbol{e}}$ LEM > 0.6 } & \multicolumn{2}{|c|}{$\overline{\boldsymbol{v}}_{\boldsymbol{e}}$ LEM > 0.7 } \\
\hline std bkg. & $2.00 \%$ & - & $2.00 \%$ & - & $2.00 \%$ & - & $2.00 \%$ & - \\
\hline$v_{\tau} \mathrm{CC}$ & $2.40 \%$ & - & $2.40 \%$ & - & $2.40 \%$ & - & $2.40 \%$ & - \\
\hline Signal & $2.40 \%$ & - & $2.40 \%$ & - & $2.40 \%$ & - & $2.40 \%$ & - \\
\hline
\end{tabular}

Table 7.5: Fractional uncertainty in the prediction of standard backgrounds, $v_{\tau} C C$, and signal events due to the normalization error.

\subsubsection{Cross Section Modeling}

Many potential sources of error due to cross section mismodeling cancel out in the Far/Near extrapolation process. A few cross section parameters, identified in (51), were selected for study as possible significant sources of systematic error in the final prediction. A $\pm 50 \%$ uncertainty is applied to the KNO parameters in the deep-inelastic scattering (DIS) cross section calculation, and $\pm 15 \%$ uncertainties are applied to the axial-vector mass term, $\mathrm{M}_{\mathrm{A}}$, for quasi-elastic (QE) and resonant (RES) interaction cross sections.

Likewise, there is a large uncertainty on the $v_{\tau}$ cross section in the MINOS Monte Carlo 
that arises from uncertainties in the pseudo-scalar form factor. ${ }^{(52)}$ This systematic error dominates the uncertainties pertaining to the $v_{\tau} \mathrm{CC}$ background prediction.

All of the cross section systematics are studied by reweighting each of the aforementioned parameters by one standard deviation and observing the change in the predictions of standard background or $v_{\tau} \mathrm{CC}$ events.

\begin{tabular}{|c|c|c|c|c|c|c|c|c|}
\hline std bkg. & \multicolumn{2}{|c|}{$v_{e}$ LEM > 0.6 } & \multicolumn{2}{|c|}{$v_{e}$ LEM > 0.7 } & \multicolumn{2}{|c|}{$\overline{\boldsymbol{v}}_{e}$ LEM > 0.6 } & \multicolumn{2}{|c|}{$\overline{\boldsymbol{v}}_{\boldsymbol{e}}$ LEM > 0.7 } \\
\hline $\mathrm{KNO}( \pm 1 \sigma)$ & $-0.03 \%$ & $0.07 \%$ & $-0.04 \%$ & $0.13 \%$ & $-0.02 \%$ & $0.11 \%$ & $-0.18 \%$ & $0.41 \%$ \\
\hline$M_{A}^{R E S}( \pm 1 \sigma)$ & $0.01 \%$ & $0.01 \%$ & $-0.01 \%$ & $0.01 \%$ & $0.32 \%$ & $-0.22 \%$ & $0.19 \%$ & $-0.12 \%$ \\
\hline$M_{A}^{Q E}( \pm 1 \sigma)$ & $-0.02 \%$ & $0.03 \%$ & $-0.01 \%$ & $0.02 \%$ & $-0.02 \%$ & $0.01 \%$ & $0.00 \%$ & $-0.00 \%$ \\
\hline$v_{\tau} \mathrm{CC}$ & \multicolumn{2}{|c|}{$v_{e}$ LEM > 0.6 } & \multicolumn{2}{|c|}{$v_{e}$ LEM $>0.7$} & \multicolumn{2}{|c|}{$\overline{\boldsymbol{v}}_{e}$ LEM $>0.6$} & \multicolumn{2}{|c|}{$\overline{\boldsymbol{v}}_{\boldsymbol{e}}$ LEM $>0.7$} \\
\hline$v_{\tau} \mathrm{xsec}$ & $44.29 \%$ & - & $45.22 \%$ & - & $47.54 \%$ & - & $43.56 \%$ & - \\
\hline
\end{tabular}

Table 7.6: Fractional uncertainty in the prediction of standard backgrounds and $v_{\tau} C C$ events due to the uncertainties in neutrino interaction cross sections.

\subsubsection{Hadronic Modeling}

Uncertainties in the AGKY hadronic modeling used by MINOS are evaluated by examining the effect six Monte Carlo weights have on the prediction. The weights are described as follows:

1. Baryon $x_{f}-$ The MC is reweighted such that the baryon momentum produces showers that bear stronger resemblance to actual interactions.

2. $\pi^{0}$ selection - The probability of $\pi^{0}$ production is shifted by $\pm 20 \%$. This particular uncertainty could have a large effect upon the LEM PID via the hadronic $y$ neural network input.

3. Multiplicity Correlation - This MC reweight simulates completely independent charged and neutral hadron multiplicities.

4. Implementation Ambiguities - Differences discovered between NEUGEN and GENIE hadron 4-vector selection processes motivated an examination of the two 
versions of AGKY. This reweight simulates a GENIE-only AGKY environment.

5. $p_{T}$ Squeezing - The $p_{T}$ rejection factor used in the KNO regime of the AGKY model is changed from 3.5 to 1.5 , resulting in broader hadronic showers. This weight simulates uncertainty in $p_{T}$ momentum of the shower.

6. Isotropic 2-Body Decay - AGKY models two-body decays isotropically in the CM frame. This reweight examines what happens when two-body decays are performed orthogonally to the direction of the momentum transfer to test the impact of the assumption.

In addition, the uncertainty on the total hadron multiplicity is evaluated and included in the total hadronic modeling systematic. Charged hadron multiplicity is parameterized as a function of the hadronic system invariant mass W. $\left(\left\langle n_{c h}\right\rangle=a+b \log W^{2}\right)$. To evaluate this uncertainty, the parameters $a$ and $b$ are shifted by the ranges permitted by bubble chamber experiments. ${ }^{(53)(54)}$ Events are then reweighted depending upon the specific neutrino-nucleon interaction.

The impact of the hadronic uncertainties upon the Far Detector predictions for various LEM intervals can be seen in Table 7.7.

\subsection{7 $v_{\mu}$ CC Spectrum}

The fiducial muon neutrino/antineutrino samples are potentially affected by a set of systematics. These uncertainties affect the flux of signal and $v_{\tau} \mathrm{CC}$ events as well as the Far/Near ratio of $v_{\mu}$ CC extrapolation, both of which demand further investigation through reweighting the relevant parameters in only the $v_{\mu}$ CC Fiducial Monte Carlo. These systematics include specific cross section uncertainties, a 50\% shift on the number 
of $\mathrm{NC}$ events selected into the $v_{\mu} \mathrm{CC}$ sample, and a $10 \%$ shift on the $\nu_{\mu} \mathrm{CC}$ energy scale.

The effects on the prediction are shown in Table 7.8.

\begin{tabular}{|c|c|c|c|c|c|c|c|c|}
\hline std bkg. & \multicolumn{2}{|c|}{$v_{e}$ LEM > 0.6} & \multicolumn{2}{|c|}{$v_{e}$ LEM $>0.7$} & \multicolumn{2}{|c|}{$\overline{\boldsymbol{v}}_{\boldsymbol{e}}$ LEM $>0.6$} & \multicolumn{2}{|c|}{$\overline{\boldsymbol{v}}_{e}$ LEM > 0.7 } \\
\hline Baryon $x_{f}$ & $-0.05 \%$ & - & $-0.19 \%$ & - & $1.59 \%$ & - & $0.27 \%$ & - \\
\hline$\pi^{0} \operatorname{sel} .( \pm 1 \sigma)$ & $-0.07 \%$ & $0.01 \%$ & $0.01 \%$ & $-0.00 \%$ & $-0.34 \%$ & $0.36 \%$ & $-0.24 \%$ & $0.24 \%$ \\
\hline mult. corr. & $0.60 \%$ & - & $0.53 \%$ & - & $0.39 \%$ & - & $0.44 \%$ & - \\
\hline imp. amb & $0.10 \%$ & - & $0.01 \%$ & - & $0.44 \%$ & - & $0.01 \%$ & - \\
\hline$p_{T}$ squz & $0.04 \%$ & - & $-0.02 \%$ & - & $0.18 \%$ & - & $-0.20 \%$ & - \\
\hline iso. 2-b. dec. & $0.18 \%$ & - & $0.13 \%$ & - & $-0.34 \%$ & - & $-0.62 \%$ & - \\
\hline Hadron Mult. & $0.37 \%$ & $0.02 \%$ & $0.09 \%$ & $-0.06 \%$ & $1.50 \%$ & $-0.21 \%$ & $1.41 \%$ & $-0.76 \%$ \\
\hline$v_{\tau} \mathrm{CC}$ & \multicolumn{2}{|c|}{$v_{e}$ LEM $>0.6$} & \multicolumn{2}{|c|}{$v_{e}$ LEM $>0.7$} & \multicolumn{2}{|c|}{$\overline{\boldsymbol{v}}_{\boldsymbol{e}} \mathrm{LEM}>0.6$} & \multicolumn{2}{|c|}{$\overline{\boldsymbol{v}}_{\boldsymbol{e}}$ LEM >0.7 } \\
\hline Hadron Mult. & $-0.63 \%$ & $-0.20 \%$ & $-0.67 \%$ & $-0.04 \%$ & $-0.38 \%$ & $-0.16 \%$ & $-0.54 \%$ & $-0.19 \%$ \\
\hline Signal & \multicolumn{2}{|c|}{$v_{e}$ LEM $>0.6$} & \multicolumn{2}{|c|}{$v_{e}$ LEM $>0.7$} & \multicolumn{2}{|c|}{$\overline{\boldsymbol{v}}_{e}$ LEM > 0.6 } & \multicolumn{2}{|c|}{$\overline{\boldsymbol{v}}_{e}$ LEM > 0.7 } \\
\hline Baryon $x_{f}$ & $-1.74 \%$ & - & $-1.86 \%$ & - & $-0.43 \%$ & - & $-0.41 \%$ & - \\
\hline$\pi^{0} \operatorname{sel} .( \pm 1 \sigma)$ & $0.61 \%$ & $-0.61 \%$ & $0.62 \%$ & $-0.61 \%$ & $0.12 \%$ & $-0.12 \%$ & $0.11 \%$ & $-0.10 \%$ \\
\hline mult. corr. & $-1.13 \%$ & - & $-1.21 \%$ & - & $-0.19 \%$ & - & $-0.19 \%$ & - \\
\hline imp. amb & $-0.52 \%$ & - & $-0.59 \%$ & - & $-0.15 \%$ & - & $-0.15 \%$ & - \\
\hline$p_{T}$ squz & $-0.36 \%$ & - & $-0.44 \%$ & - & $-0.12 \%$ & - & $-0.12 \%$ & - \\
\hline iso. 2-b. dec. & $-0.39 \%$ & - & $-0.67 \%$ & - & $-0.11 \%$ & - & $-0.17 \%$ & - \\
\hline Hadron Mult. & $-1.31 \%$ & $-0.97 \%$ & $-1.39 \%$ & $-0.90 \%$ & $0.03 \%$ & $-0.45 \%$ & $0.01 \%$ & $-0.45 \%$ \\
\hline
\end{tabular}

Table 7.7: Fractional uncertainty in the prediction of standard backgrounds, $v_{\tau} C C$, and signal events due to hadronic modeling systematics.

\begin{tabular}{|c|c|c|c|c|c|c|c|c|}
\hline$v_{\tau} \mathrm{CC}$ & \multicolumn{2}{|c|}{$v_{e}$ LEM $>0.6$} & \multicolumn{2}{|c|}{$v_{e}$ LEM > 0.7 } & \multicolumn{2}{|c|}{$\overline{\boldsymbol{v}}_{\boldsymbol{e}}$ LEM > 0.6} & \multicolumn{2}{|c|}{$\overline{\boldsymbol{v}}_{\boldsymbol{e}} \mathbf{L E M}>0.7$} \\
\hline$v_{\mu} \mathrm{KNO}( \pm 1 \sigma)$ & $-0.26 \%$ & $0.14 \%$ & $-0.26 \%$ & $0.14 \%$ & $-0.11 \%$ & $0.05 \%$ & $-0.11 \%$ & $0.05 \%$ \\
\hline$v_{\mu} M_{A}^{R E S}( \pm 1 \sigma)$ & $-0.58 \%$ & $0.68 \%$ & $-0.58 \%$ & $0.68 \%$ & $-0.13 \%$ & $0.19 \%$ & $-0.13 \%$ & $0.19 \%$ \\
\hline$\nu_{\mu} M_{A}^{Q E}( \pm 1 \sigma)$ & $-0.36 \%$ & $0.36 \%$ & $-0.36 \%$ & $0.35 \%$ & $-0.04 \%$ & $0.05 \%$ & $-0.04 \%$ & $0.05 \%$ \\
\hline CC E. Shift & $-1.18 \%$ & $1.48 \%$ & $-1.25 \%$ & $1.56 \%$ & $-0.95 \%$ & $1.09 \%$ & $-0.97 \%$ & $1.10 \%$ \\
\hline CC Sig NC & $-0.60 \%$ & $0.61 \%$ & $-0.60 \%$ & $0.61 \%$ & $-0.53 \%$ & $0.53 \%$ & $-0.53 \%$ & $0.53 \%$ \\
\hline Signal & \multicolumn{2}{|c|}{$v_{e}$ LEM > 0.6 } & \multicolumn{2}{|c|}{$v_{e}$ LEM > 0.7 } & \multicolumn{2}{|c|}{$\overline{\boldsymbol{v}}_{\boldsymbol{e}}$ LEM $>0.6$} & \multicolumn{2}{|c|}{$\bar{v}_{e}$ LEM > 0.7 } \\
\hline$v_{\mu} \mathrm{KNO}( \pm 1 \sigma)$ & $0.30 \%$ & $-0.44 \%$ & $0.30 \%$ & $-0.44 \%$ & $0.17 \%$ & $-0.19 \%$ & $0.17 \%$ & $-0.20 \%$ \\
\hline$v_{\mu} M_{A}^{R E S}( \pm 1 \sigma)$ & $0.29 \%$ & $-0.21 \%$ & $0.29 \%$ & $-0.21 \%$ & $0.45 \%$ & $-0.05 \%$ & $0.46 \%$ & $-0.05 \%$ \\
\hline$v_{\mu} M_{A}^{Q E}( \pm 1 \sigma)$ & $-0.11 \%$ & $0.19 \%$ & $-0.11 \%$ & $0.19 \%$ & $-0.04 \%$ & $0.17 \%$ & $-0.04 \%$ & $0.17 \%$ \\
\hline CC E. Shift & $3.54 \%$ & $-2.70 \%$ & $3.51 \%$ & $-2.66 \%$ & $4.31 \%$ & $-3.49 \%$ & $4.34 \%$ & $-3.51 \%$ \\
\hline CC Sig NC & $-0.54 \%$ & $0.55 \%$ & $-0.54 \%$ & $0.55 \%$ & $-0.40 \%$ & $0.41 \%$ & $-0.40 \%$ & $0.41 \%$ \\
\hline
\end{tabular}

Table 7.8: Fractional uncertainty in the prediction of $v_{\tau} C C$ and signal events due to $v_{\mu}$ CC spectrum systematics. 


\subsubsection{RHC Investigation}

Since the RHC sample contains a significant neutrino contamination, an additional systematic was evaluated to ascertain how well the simulation modeled antineutrino vs. neutrino flux. This uncertainty was studied by using 3000 pseudo-experiments provided by the beam systematics group, in which randomly-distributed beam parameters and varying $\pi^{+} / \pi^{-}$ratios (hence varying $v / \bar{v}$ ratios) were applied. The antineutrino vs. neutrino flux was allowed to fluctuate over $\mathrm{a} \pm 1 \sigma$ window in this simulation.

One-dimensional $(\mathrm{F} / \mathrm{N})_{\text {shifted }}$ histograms, binned in terms of the LEM PID and reconstructed energy, were generated for all pseudo-experiments and standard background types by using energy distributions taken from the standard analysis Monte Carlo. These histograms were then used to generate $\mathrm{F} / \mathrm{N}$ covariance matrices ( filled as $\operatorname{Cov}(x, y) / \bar{x} \bar{y})$. The uncertainty was set by using the maximum diagonal element of each matrix, as $\operatorname{Cov}(x, x) / \bar{x} \bar{x}=\sigma^{2} / \bar{x}^{2}$. The errors assigned to the $\mathrm{F} / \mathrm{N}$ ratios for each standard background type are shown in Table 7.9. The total effect on the background prediction was found to be less than $0.5 \%$.

\begin{tabular}{|c|c|}
\hline Background & F/N Uncertainty \\
\hline $\mathrm{NC}$ & $0.42 \%$ \\
\hline$v_{\mu} \mathrm{CC}$ & $0.51 \%$ \\
\hline Beam $v_{e}$ & $0.80 \%$ \\
\hline
\end{tabular}

Table 7.9: Uncertainties evaluated for each background component when the antineutrino vs. neutrino flux is varied within a $\pm 1 \sigma$ window. 


\subsection{Total Uncertainties}

Table 7.10 shows the total uncertainty in the Far Detector prediction for background, $v_{\tau}$ $\mathrm{CC}$, and signal events for listed regions of the neutrino and antineutrino LEM PID. With the systematics understood, we have a basis to determine the significance of our result.

\begin{tabular}{|c|r|r|r|r|}
\hline Tot. Extrap. & \multicolumn{2}{|c|}{$\boldsymbol{v}_{\boldsymbol{e}}$ LEM $>\mathbf{0 . 6}$} & \multicolumn{2}{|c|}{$\boldsymbol{v}_{\boldsymbol{e}}$ LEM $>\mathbf{0 . 7}$} \\
\hline std bg. & $3.57 \%$ & $-3.41 \%$ & $4.47 \%$ & $-3.77 \%$ \\
\hline$v_{\tau}$ CC & $44.87 \%$ & $-44.78 \%$ & $46.35 \%$ & $-46.24 \%$ \\
\hline Signal & $5.53 \%$ & $-5.26 \%$ & $6.69 \%$ & $-6.90 \%$ \\
\hline- & \multicolumn{2}{|c|}{$\overline{\boldsymbol{v}}_{\boldsymbol{e}}$ LEM $>\mathbf{0 . 6}$} & \multicolumn{2}{|c|}{$\overline{\boldsymbol{v}}_{\boldsymbol{e}}$ LEM $>\mathbf{0 . 7}$} \\
\hline std bg. & $4.39 \%$ & $-3.88 \%$ & $4.06 \%$ & $-3.52 \%$ \\
\hline$v_{\tau} \mathrm{CC}$ & $43.26 \%$ & $-43.37 \%$ & $44.52 \%$ & $-44.50 \%$ \\
\hline Signal & $5.47 \%$ & $-5.03 \%$ & $6.01 \%$ & $-5.72 \%$ \\
\hline
\end{tabular}

Table 7.10: Total systematic uncertainty on FHC and RHC Far Detector predictions, given the labeled PID range. 


\section{Chapter 8}

\section{Far Detector Prediction}

With the Near Detector beam decomposition described and systematics understood, the tools are in place to make the Far Detector prediction. The extrapolation receives input oscillation parameters based on experimental global averages, and predictions are made for five neutrino interaction types: $\mathrm{NC}, v_{\mu} \mathrm{CC}$, beam $v_{e}, v_{\tau} \mathrm{CC}$, and signal $v_{e} \mathrm{CC}$ events. Antineutrino counterparts to the charged current interactions are considered for the RHC portion of the analysis.

\subsection{NC, $v_{\mu} \mathrm{CC}$, and Beam $v_{e}$ Backgrounds}

These three Far Detector predictions are made using the HOOHE Near Detector beam decomposition method. This technique determines the rates at which each background type occurs in the data sample. Once again, the Far Detector prediction is calculated in each analysis bin for each background as shown in the expression below.

$$
\operatorname{Far}_{b c, i}^{\text {Prediction }}=\operatorname{Near}_{b c, i}^{\text {Data }} \times\left(\operatorname{Far}_{b c, i}^{\mathrm{MC}} / \operatorname{Near}_{b c, i}^{\mathrm{MC}}\right)
$$

Several effects necessitate the component separation to make the prediction. The $v_{\mu} \mathrm{CC}$ rate, for example, will be affected by the oscillation phenomenon in a manner that produces a Far Detector deficit, while the NC rate will remain unaltered by oscillation physics. In addition, beam $v_{e}$ events arise from secondary muon decays, which have different angular distributions from their $v_{\mu} \mathrm{CC}$ brethren because they occur farther down the beam pipe. All these features produce different energy spectra for each background 
component that requires extrapolation to be done separately.

\begin{tabular}{|c|c|c|}
\hline Background & $\boldsymbol{v}$ Sample & $\overline{\boldsymbol{v}}$ Sample \\
\hline $\mathrm{NC}$ & 89.4 & 13.9 \\
\hline$v_{\mu} \mathrm{CC}+\bar{v}_{\mu} \mathrm{CC}$ & 21.6 & 1.0 \\
\hline Beam $v_{e}+\bar{v}_{e}$ & 11.9 & 1.8 \\
\hline
\end{tabular}

Table 8.1: Far Detector predictions in the LEM $>0.6$ region for the three main backgrounds in both the FHC (left) and RHC (right) configurations, assuming normal mass hierarchy, $\delta_{C P .}=0$, $\sin ^{2}\left(2 \theta_{13}\right)=0.1$, and $\theta_{23}=\pi / 4$.

\section{$8.2 v_{\tau}$ CC and Signal $v_{e}$ CC Predictions}

Predicting the three backgrounds mentioned in 8.1 becomes a relatively trivial exercise since both Near and Far Detector data exist and the HOOHE data-driven method facilitates vetted extrapolation. However, both $v_{\tau} \mathrm{CC}$ and signal $v_{e} \mathrm{CC}$ interactions are the result of neutrino oscillations and thus have no Near Detector component with which to make a prediction using the standard prescription. Instead, the fiducial $\nu_{\mu} \mathrm{CC}$ spectrum is used to create the $v_{\tau} \mathrm{CC}$ and signal $v_{e} \mathrm{CC}$ predictions. This occurs with the following procedure:

1. The reconstructed $v_{\mu}$ spectrum is transformed into the true $v_{\mu}$ spectrum by correcting the Far Detector $v_{\mu}$ CC prediction with a two-dimensional, reconstructed-true energy transformation matrix, RT. The spectrum is additionally corrected for the purity, $\rho$, and efficiency, $\epsilon$, of the $v_{\mu} \mathrm{CC}$ selection. The expression looks as follows:

$$
F_{t}^{v_{\mu} \mathrm{CC}}=\left[\sum_{r}\left[\frac{F_{r}^{\mathrm{MC}}}{N_{r}^{\mathrm{MC}}} N_{r}^{\mathrm{Data}}\right] R T_{r t}\right] \frac{\rho_{t}}{\epsilon_{t}}
$$

where $t$ is a bin of true energy, $r$ is a bin of reconstructed energy, and $F_{r}$ and $N_{r}$ are the CC selections in reconstructed energy $r$ for either a Far or Near Detector sample. Data and MC quantities are designated with superscripts. 
2. The true $v_{\mu}$ spectrum is converted into a true $v_{l}$ spectrum by reweighting Expression (8.1) by the probability of $v_{\mu} \rightarrow v_{l}$ oscillation and scaling to the necessary cross section of the desired leptonic interaction. 8.2 yields the predicted true energy $v_{l}$ spectrum in the Far Detector fiducial region.

$$
F_{t}^{v_{l} C C}=F_{t}^{v_{\mu} C C} P_{t}\left(v_{\mu} \rightarrow v_{l}\right) \frac{\sigma_{t}^{l}}{\sigma_{t}^{\mu}}
$$

3. The true $v_{l}$ spectrum is converted back into a reconstructed energy spectrum by applying a second transformation matrix, TR. To convert this quantity to an analysis ready prediction, an additional factor is introduced that represents the cumulative selection efficiency of the analysis preselection and bin $p$ of the PID algorithm for Far Detector $v_{l} \mathrm{CC}$ interactions. The resulting output is a prediction of the number of $v_{l}$ events in reconstructed energy bin $r$ and LEM PID bin $p$.

$$
F_{p r}^{v_{l} C C}=\sum_{t} F_{t}^{v_{l} C C} T R_{r t} \varepsilon_{p r}^{v_{l}}
$$

where $\varepsilon$ is the cumulative selection efficiency for $v_{l}$ interactions in the specified analysis bin.

\begin{tabular}{|c|c|c|}
\hline Osc. Samples & $\boldsymbol{v}$ Sample & $\overline{\boldsymbol{v}}$ Sample \\
\hline$v_{\tau} \mathrm{CC}+\bar{v}_{\tau} \mathrm{CC}$ & 4.8 & 0.8 \\
\hline$v_{\mu} \rightarrow v_{e} \mathrm{CC}$ & 33.0 & 0.7 \\
\hline $\bar{v}_{\mu} \rightarrow \bar{v}_{e} \mathrm{CC}$ & 0.7 & 3.2 \\
\hline
\end{tabular}

Table 8.2: Far Detector predictions in the LEM $>0.6$ region for oscillation events in both the FHC (left) and RHC (right) configurations, assuming normal mass hierarchy, $\delta_{C P .}=0, \sin ^{2}\left(2 \theta_{13}\right)=0.1$, and $\theta_{23}=\pi / 4$. Note that if we assumed $\sin ^{2}\left(2 \theta_{13}\right)=0$, the bottom two rows would have zero predicted events.

\subsection{Selection Efficiency Correction}

Additional considerations are made in regards to the $\varepsilon_{p r}^{v_{e}}$ term from Expression (8.3) for 
$v_{e}$ CC Monte Carlo events. This quantity is purely MC-derived, and it is thus susceptible to unwanted shifts due to mismodeling of the hadronic component of $\mathrm{MC} v_{e} \mathrm{CC}$

interactions. As is the case with many of the techniques adopted by the appearance group, we seek to employ a data-incorporating method that can correct for any MC mismodeling.

Since the signal region is blinded, however, the actual data cannot be used to derive the correction to the selection efficiency. Instead, we use what is called the Muon Removed, Electron added (MRE) sample. This sample is formed by taking $v_{\mu} \mathrm{CC}$ events with welldefined muon tracks. The muon is removed from the event, leaving a hadronic shower, and the muon's energy, momentum, and vertex position are used to add a simulated electron shower to the interaction. These MRE events then proceed through the standard reconstruction and analysis chains, and the resulting collection of events serves as a replacement signal sample that does not encroach upon the blinded data.

MRE events are produced using both Near Detector Data and Monte Carlo, and the selection efficiencies of various PID bins (relative to the fiducial cut level) are determined. The ratio of the Data and MC efficiencies is used as the correction factor for the term in Expression (8.3).

\begin{tabular}{|c|c|c|}
\hline PID Region & $\boldsymbol{v}$ Sample & $\overline{\boldsymbol{v}}$ Sample \\
\hline $0.6-0.7$ & 0.97 & 0.97 \\
\hline $0.7-0.8$ & 0.97 & 0.96 \\
\hline $0.8-1.0$ & 1.00 & 0.95 \\
\hline
\end{tabular}

Table 8.3: Efficiency correction factors, $C_{M R E}$, for both the FHC (left) and RHC (right) samples. Factors were calculated by comparing the number Near Detector MRE Data and MC events in each LEM PID bin to the number of events in the respective fiducial MRE samples. ${ }^{(40)(55)(56)}$ 


\subsection{Near Detector Results \& Making the Far Detector Prediction}

With all of the tools in place, the Far Detector prediction can be made for background and signal components with specified oscillation parameters. This section will present the parameters used to make the prediction along with plots that show the results of the Near Detector beam decomposition, the Near Detector PID response, and the Far Detector prediction. Eventually, contours for both mass hierarchies that span all possible values of $\delta_{\mathrm{CP}}$ will be generated (see Fig. 5.1) to determine the confidence intervals of the $\theta_{13}$ parameter. For now, the prediction is calculated using the values for the oscillation parameters specified below to give a general indication as to what is expected at the Far Detector.

1. $\theta_{23}=\pi / 4-$ Maximal atmospheric mixing is assumed. This is consistent with the $\sin ^{2}\left(2 \theta_{23}\right)>0.95$ at $90 \%$ C.L. result from Super-Kamiokande. ${ }^{(15)}$

2. $\theta_{12}=0.594-$ Source (57).

3. $\theta_{13}=0.16-$ Value is consistent with $\sin ^{2}\left(2 \theta_{13}\right)=0.1$, which is within the current reactor-based experimental constraints.

4. $\Delta m_{21}^{2}=7.59 \times 10^{-5} \mathrm{eV}^{2}-$ Source (15).

5. $\Delta m_{32}^{2}=2.39 \times 10^{-3} \mathrm{eV}^{2}-$ Value consistent with the 2012 MINOS disappearance analysis. ${ }^{(58)}$ This value is defined as positive to make the prediction with the normal hierarchy assumption.

6. $\delta_{\mathrm{CP}}=0-$ There are currently no definitive limits on $\delta_{\mathrm{CP}}$. For extrapolation purposes, this parameter is set to zero. 


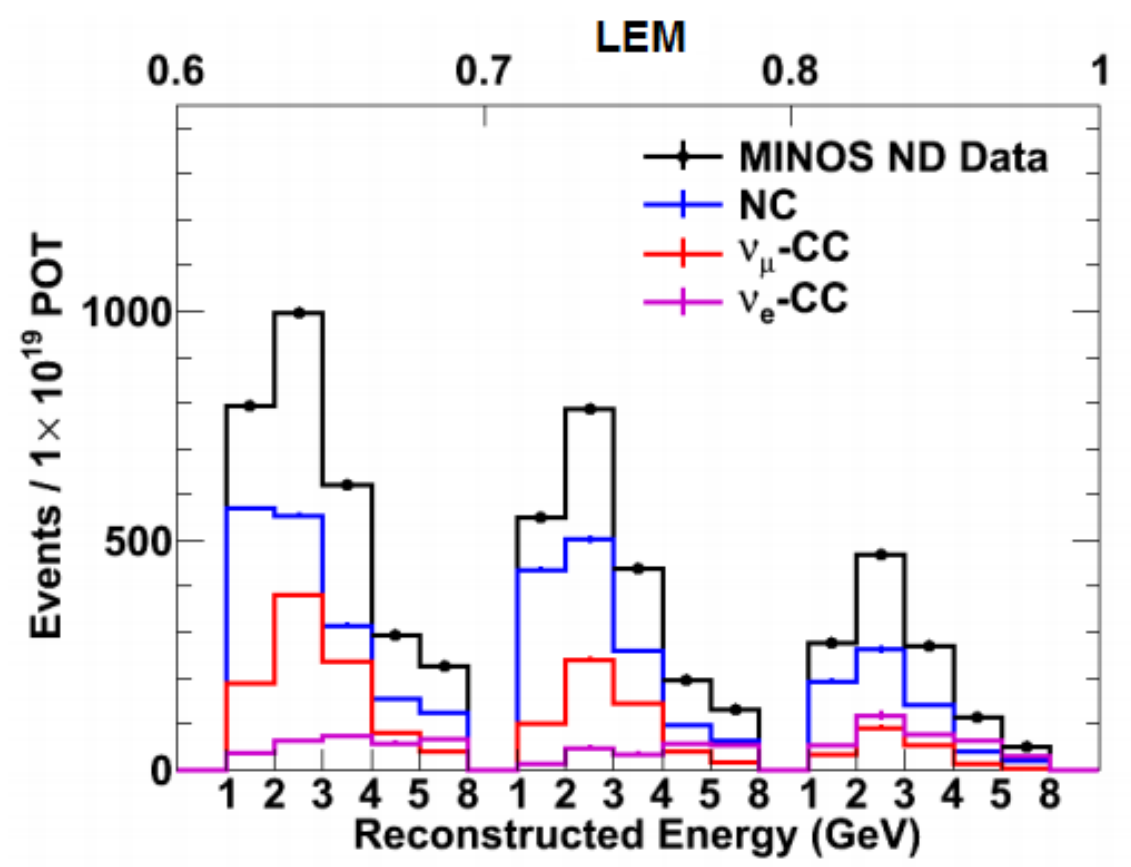

Figure 8.1: Results of the Near Detector beam decomposition binned in terms of reconstructed energy and LEM PID for Set I (FHC Runs I, II, III, IV, V, and VI).

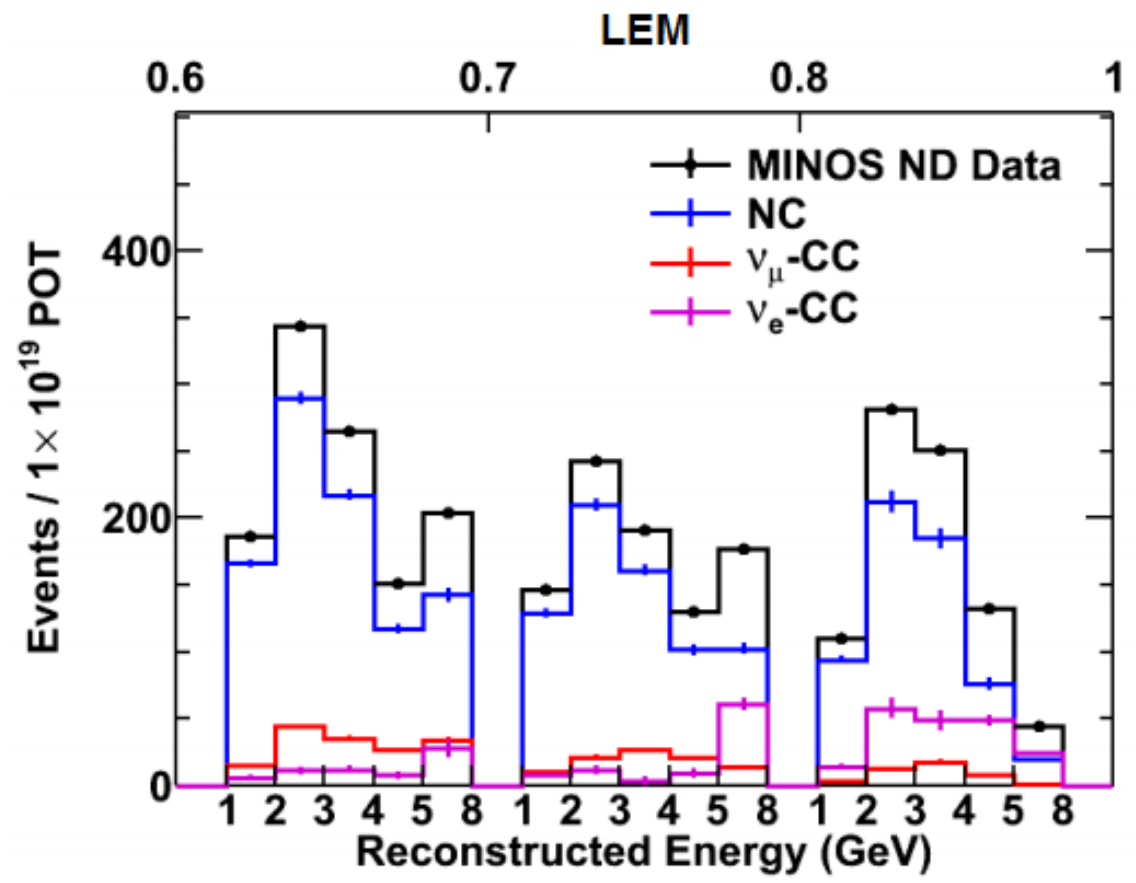

Figure 8.2: Results of the Near Detector beam decomposition binned in terms of reconstructed energy and LEM PID for Set II (RHC Runs IV, VII, and IX). 


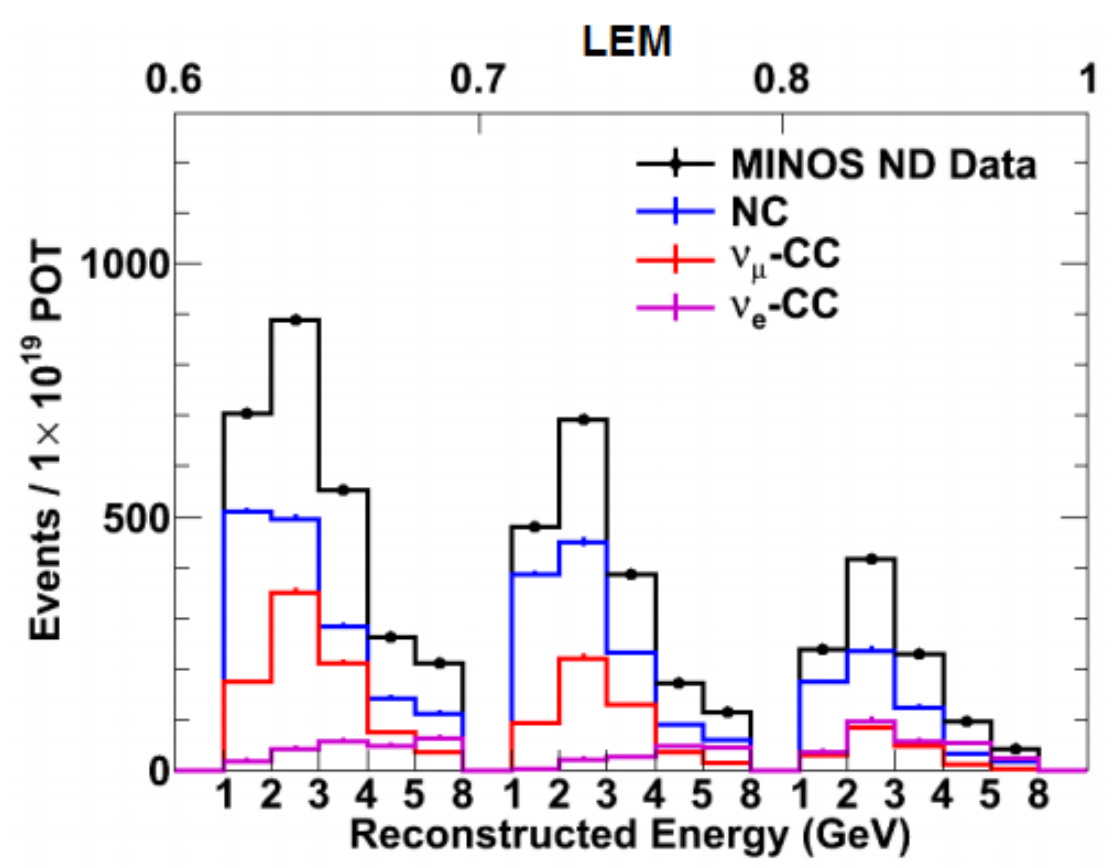

Figure 8.3: Results of the Near Detector beam decomposition binned in terms of reconstructed energy and LEM PID for Set III (FHC Runs VIII and X).

As mentioned at the beginning of this chapter, the Near Detector beam decomposition represents the first part in making the Far Detector prediction by separating the three main background components for extrapolation. Most of the code developed for the appearance analysis was designed to handle three run periods. To simplify the transition to a larger data set, Sets I-III were created. Hence, the decomposition was performed for each of the three sets using the HOOHE data-driven method described in Section 5.2.3 shown, and the results are shown in the three previous figures.

The following two figures show the predicted Far Detector reconstructed energy spectrum for both the joint $\mathrm{FHC}+\mathrm{RHC}$ sample and the RHC sample alone. The plots were generated using the methods outlined in the previous sections of this chapter. 

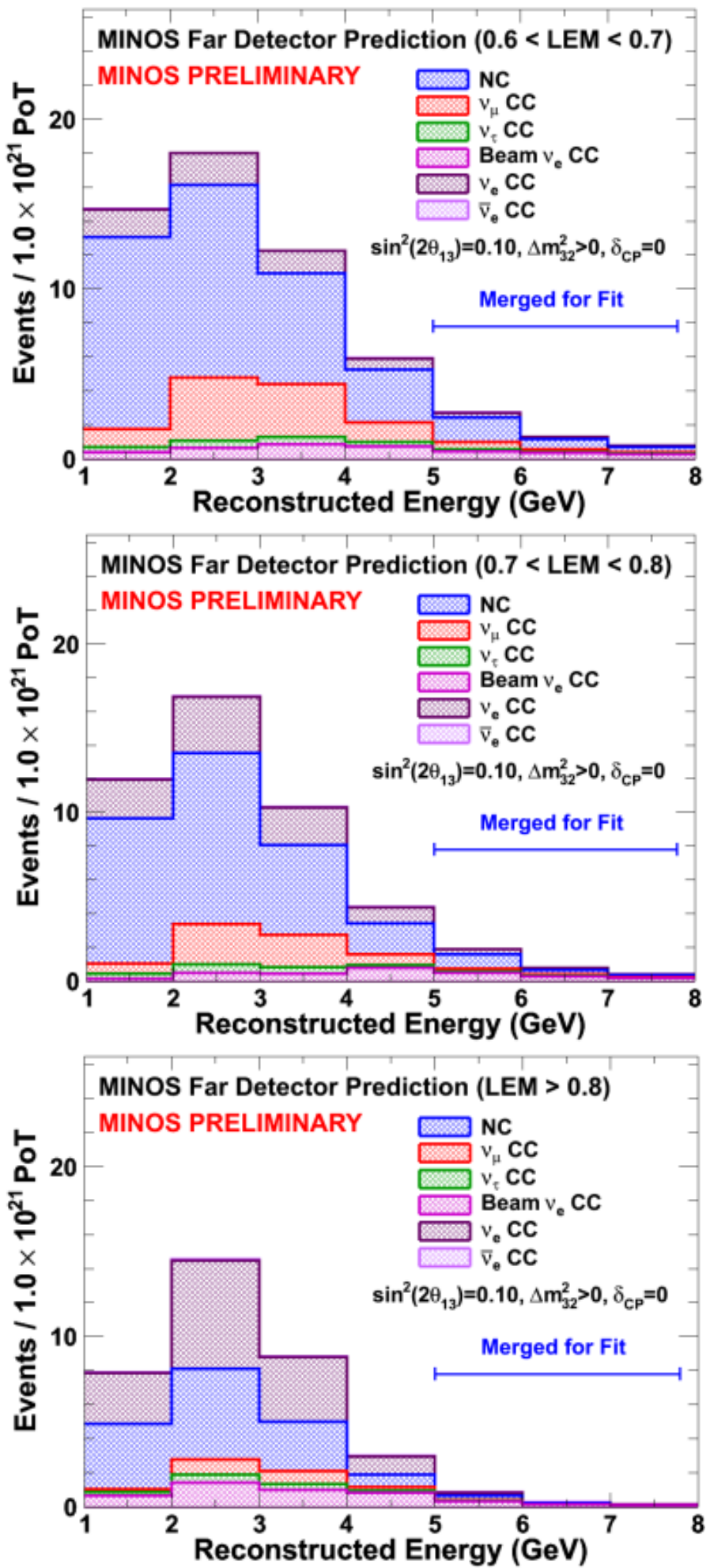

Figure 8.4: Stacked histograms of the predicted FHC $+R H C$ Far Detector spectrum in the defined LEM PID regions. Events normalized to $1.0 \times 10^{21}$ POT. Images from (40). 

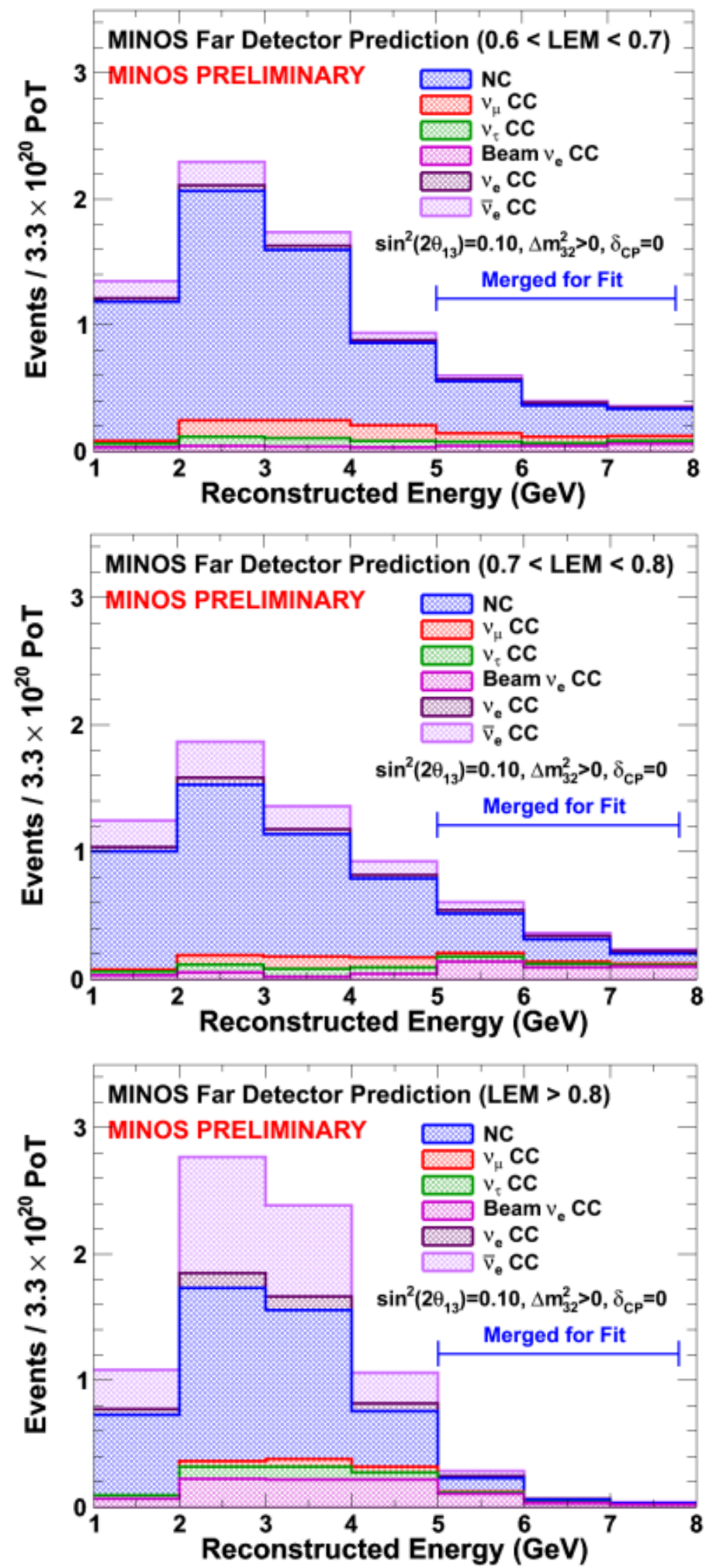

Figure 8.5: Stacked histograms of the predicted RHC Far Detector spectrum in the defined LEM PID regions. Events normalized to $3.3 \times 10^{20}$ POT. Images from (39). 
In addition to the reconstructed energy distribution, the Far Detector prediction can be represented as a distribution of the LEM PID. The figure below shows actual Near Detector PID distributions for both neutrino and antineutrino modes as well as the predicted Far Detector PID histograms.
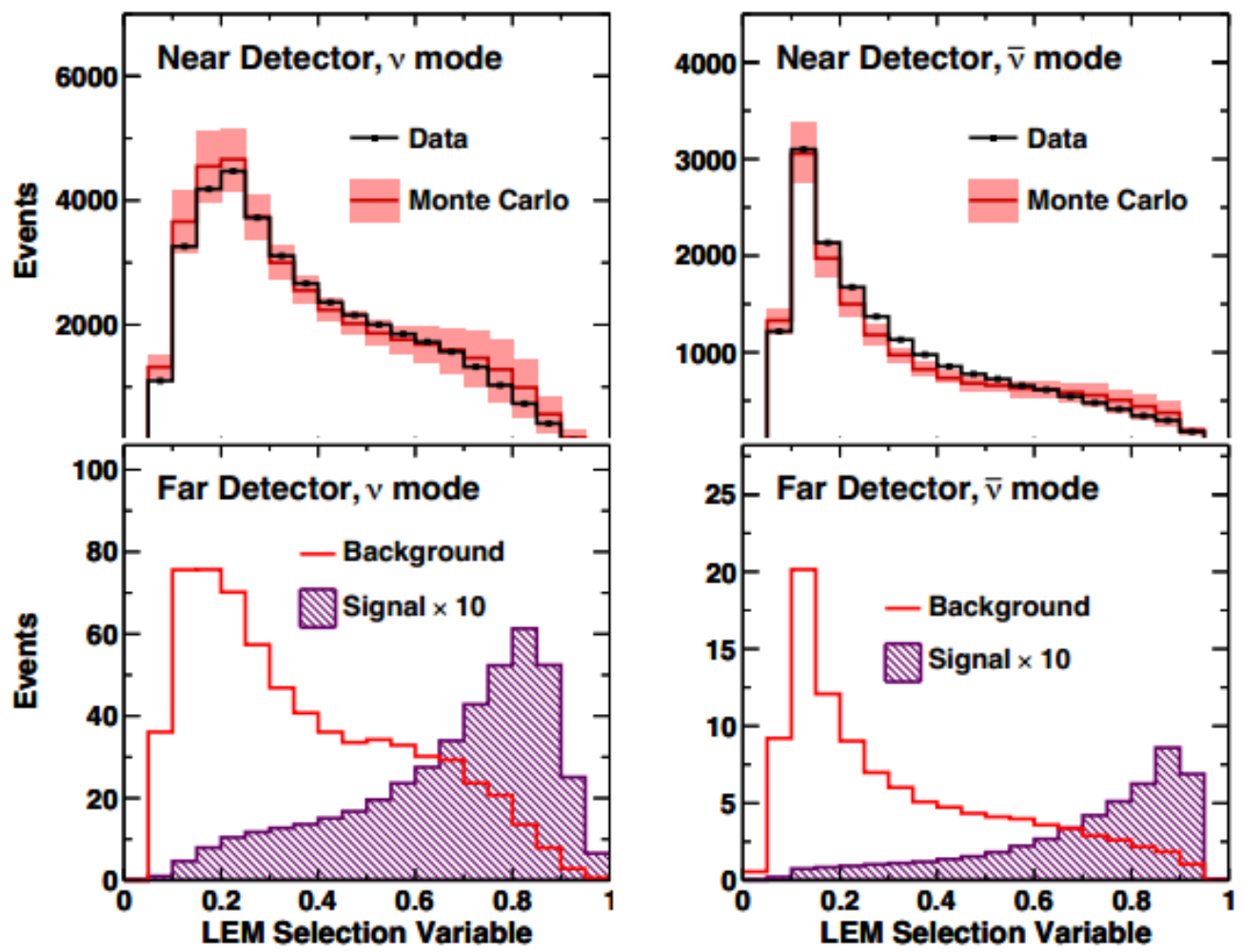

Figure 8.6: LEM PID distributions of the FHC sample (left) and the RHC sample (right). The top row of plots contains the distributions of Near Detector preselection events. The bands associated with the MC distributions represent the calculated systematic errors. The bottom row of plots contains the predicted Far Detector PID distributions for both background and signal events. Images from (59).

Using these techniques - and with the parameter definitions specified in this section - we predict 161.4 (127.7 background) events in the FHC sample and 21.4 (17.5 background) events in the RHC sample with LEM PID values $>0.6 .{ }^{(59)}$

In the signal-rich LEM $>0.7$ region, we expect $69.1 \pm 8.3_{\text {stat }} \pm 4.3_{\text {syst }}$ background events in the FHC mode and $10.5 \pm 3.2_{\text {stat }} \pm 0.6_{\text {syst }}$ background events in the RHC mode. ${ }^{(27)}$ 
The fit procedure, explained in the following chapter, makes use of the LEM PID $>0.6$

region. For counting experiment purposes, the LEM PID $>0.7$ range will be referenced in Chapter 10 . 


\section{Chapter 9}

\section{Fitting Procedures for the Far Detector Data}

Many of the fitting procedures used in this thesis were developed for the 2011 appearance search. Prior to the incorporation of this method, the first appearance analyses relied upon simple counting experiments to compare the observed data with the prediction. The multi-bin shape fit allows us to extract as much information as possible from our samples. This chapter will describe the fit methodology as well as the manner in which systematic errors were incorporated into the process.

\subsection{Fit Methodology}

Typically, one could perform a fit by minimizing $\chi^{2}$. This approach, however, is not valid for the appearance analyses because of the small statistics expected in the Far Detector background and signal samples. Instead, we use a likelihood method based in Poisson statistics in which the log-likelihood is defined as follows:

$$
-\ln \mathcal{L}=\sum_{i} \ln \left(n_{i} !\right)+\mu_{i}-n_{i} \ln \mu_{i}
$$

where $n$ is the observed number of events in analysis bin $i$ and $\mu$ is the predicted number of events in bin $i$.

Similar to the LEM matching procedure, the self-likelihood (when $\mu_{i}=n_{i}$ ) is subtracted from Expression (9.1) to produce a likelihood ratio.

$$
-\ln \mathcal{L}=\sum_{i} \mu_{i}-n_{i}+n_{i} \ln n_{i}-n_{i} \ln \mu_{i}
$$

The benefit of (9.2) is that one can take $-2 \ln \mathcal{L}$ and find that it converges to $\chi^{2}$ in the high-statistics limit. Because of this feature, the fit is performed by finding the parameters that minimize $-2 \ln \mathcal{L}$ as derived from (9.2). 
The $n_{i}$ parameter will remain constant during the fitting procedure as it is pulled from actual data. The expected number of events in a bin, $\mu_{i}$, will change as $\theta_{13}$ is incremented over a specified region. $-2 \ln \mathcal{L}$ is calculated at various increments of $\theta_{13}$ for a particular value of $\delta_{\mathrm{CP}}$. The minimum value dictates where the best fit point on the $\sin ^{2} 2 \theta_{13}$ axis resides, and the region bound by $(-2 \ln \mathcal{L})-(-2 \ln \mathcal{L})_{\min }=-2 \Delta \ln \mathcal{L} \leq 2.71$ dictates the $90 \%$ C.L. ${ }^{(15)}$

The lower bound of this interval poses a particular problem, however. Gaussian intervals near physical boundaries - in this case provided by $\theta_{13}=0$ - are notorious for yielding incorrect confidence ranges. The solution adopted by the appearance group is to use the Feldman-Cousins prescription to compute more reliable confidence intervals. ${ }^{(60)}$ More information about this method will be provided in Section 9.3.

\subsection{Incorporating Systematics}

In Chapter 7, systematic errors were presented by examining the effects on the Far Detector prediction given a LEM PID range. While this is a convenient way to express the significance of the systematic uncertainties present in the appearance analyses, it does not convey the process through which those uncertainties were included in the fit. The fit statistics are Poisson as the data is discrete in nature; however, the systematics may be considered Gaussian. Hence, we incorporate the systematic uncertainties into the fit through a covariance matrix, $\mathrm{V}$.

$$
V_{i j}=\sum_{k} \delta_{i}^{k} \delta_{j}^{k}
$$

where $\delta_{i}^{k}$ is the change in the Far Detector prediction in analysis bin $i$ given a $\sigma$ shift in systematic $k$. The log-likelihood expression incorporates this covariance matrix in the 
following manner:

$$
-2 \ln \mathcal{L}=2 \sum_{i}\left[\mu^{\prime}{ }_{i}-n_{i}+n_{i} \ln n_{i}-n_{i} \ln \mu_{i}^{\prime}\right]+f^{T} V^{-1} f
$$

where $\mu_{i}^{\prime}=\mu_{i}+f_{i}$, and $f_{i}$ are nuisance parameters introduced for each analysis bin. The nuisance parameters are determined during the minimization process of Expression (9.4) using the MINUIT package. ${ }^{(61)}$ This method is susceptible to two damaging side-effects. If too many systematics are incorporated into the covariance matrix, then $\mathrm{V}$ can be almost singular, which results in complications during numerical inversion. Additionally, if the binning scheme of the analyses becomes large, then the processing time of this minimization technique increases, and there is an increased probability that the matrix inversion will produce difficulties. In the previous analysis, this method was selected to handle 45 systematic errors with 15 analysis bins. ${ }^{(48)}$ The same binning scheme was chosen for this thesis, and the number of systematics was reduced (as mentioned in Chapter 7). Therefore, this minimization method was once again selected to incorporate the systematic uncertainties into the fits of the oscillation parameters.

\subsection{Feldman-Cousins Prescription}

To avoid the inaccuracies associated with confidence level calculations near physical boundaries, the Feldman-Cousins method is implemented. This technique works by computing a new C.L. for a given $-2 \Delta \ln \mathcal{L}_{\text {Data }}$ using generated pseudo-experiments. The process is outlined below.

1. Generate 10,000 15-tuples of correlated Gaussian random variables with the means set by the Far Detector predictions for the 15 analysis bins at the particular $\theta_{13}-\delta_{\mathrm{CP}}$ grid point in question. The covariance from $\mathrm{V}$ at this grid point is also fed into the generation. 
2. Generate 10,000 15-tuples with Poisson-distributed random variables using the elements of the step one 15-tuples as the means of the new elements. These 10,000 15tuples are the pseudo-experiments, which represent possible data outcomes observed in the Far Detector with predefined true parameters set by the location of the $\theta_{13}-\delta_{\mathrm{CP}}$ grid. 3. Compute $-2 \Delta \ln \mathcal{L}_{p s e}$ at the current grid point for all pseudo-experiments, where the difference is between the minimum value of $-2 \ln \mathcal{L}$ along the $\theta_{13}$-related axis and the value of $-2 \ln \mathcal{L}$ at the selected grid point. The C.L. is determined by finding the fraction of pseudo-experiments for which $-2 \Delta \ln \mathcal{L}_{\text {pse }}<-2 \Delta \ln \mathcal{L}_{\text {Data }}$.

The $68 \%$ and $90 \%$ Feldman-Cousins confidence levels contours are generated for both of the possible mass hierarchies and span all values of $\delta_{\mathrm{CP}}$. With this technique, we can ascertain the proper probability coverage that standard Gaussian levels do not provide near physical boundaries. 


\section{Chapter 10}

\section{Far Detector Data \& Results}

The previous chapters have detailed the methods developed to perform the appearance analyses presented for this thesis. With a handling on the systematic errors, a developed fitting technique, and the means to address probability coverage near physical boundaries, the tools are in place to look at the Far Detector data and interpret results from that information.

\subsection{Sidebands}

Before the events in the signal region are examined, we validate our analysis techniques using other available data sets. The results of these checks, described in Section 5.2.6, will be presented in this section. The Anti-PID region is arguably the best candidate for a sideband analysis, for it is defined as a signal exclusive region that is unused in the final fit and has already undergone the extrapolation process. Specifically, events that make up the Anti-PID sideband have LEM PID values less than 0.5. Just as the Far Detector prediction can be made for the binning scheme chosen to optimize signal selection sensitivity, it can be performed for LEM bins spanning low PID values. This prediction is compared against the LEM $<0.5$ region in the Far Detector data. A better than $\pm 1 \sigma$ agreement between the prediction and the data is ideal, and it indicates that our extrapolation methods behave as expected.

Using the same oscillation parameters in the extrapolation as defined in Section 8.4, $458.4 \pm 21.4$ events are expected in the FHC Anti-PID sample. 475 events are observed in the LEM $<0.5$ FHC Far Detector data, yielding a $0.78 \sigma$ discrepancy. In the RHC Anti- 
PID sample, $78.6 \pm 8.9$ Far Detector events are expected. 84 events are observed in the LEM $<0.5$ RHC Far Detector data, yielding a $0.61 \sigma$ discrepancy. The Anti-PID checks showed agreement between the Far Detector data and predictions for both neutrino and antineutrino modes. The PID distributions are displayed in Figures 10.1 and 10.2.

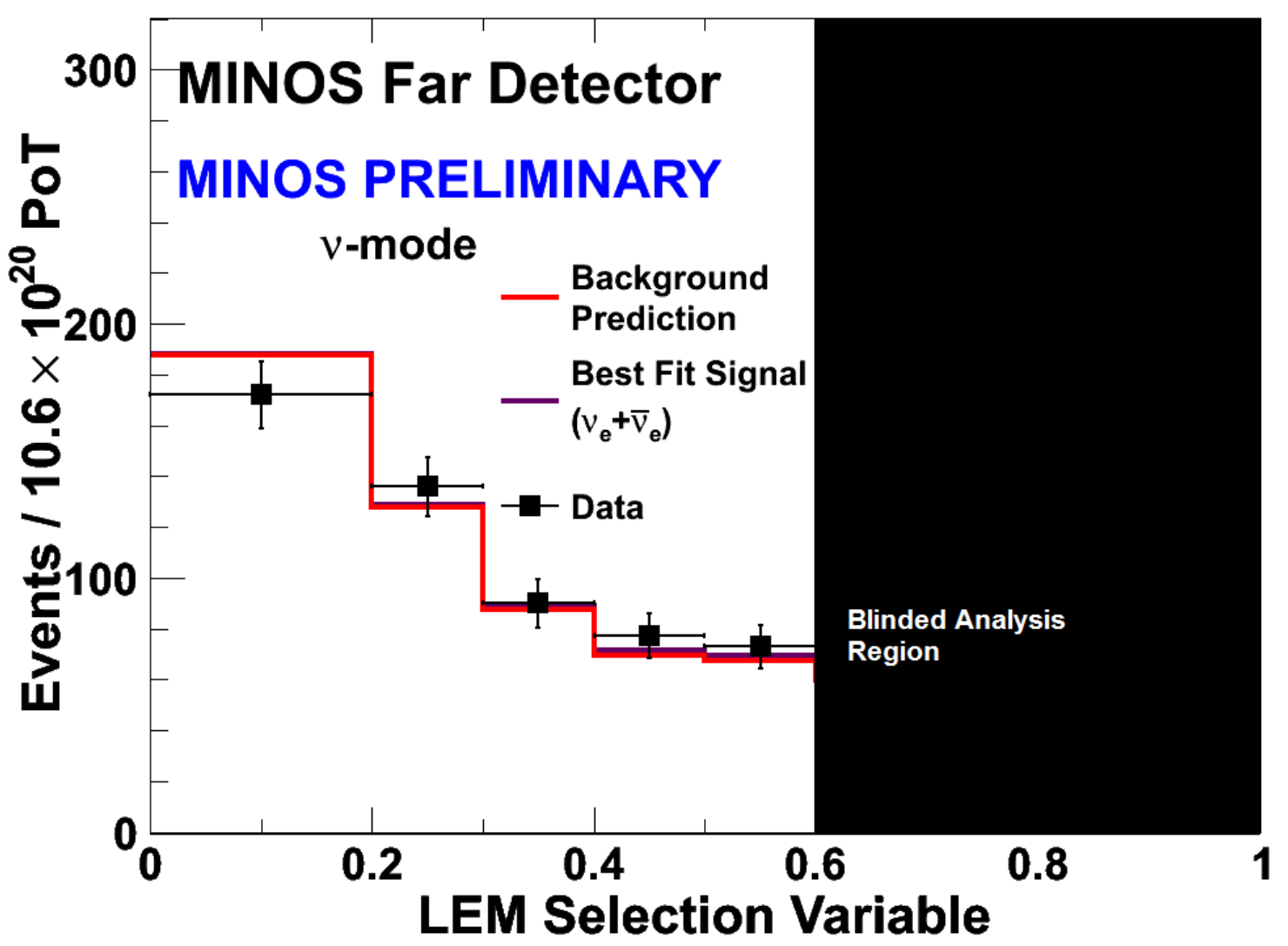

Figure 10.1: LEM PID distribution in the FHC Anti-PID sideband for the Far Detector prediction and data. Oscillation parameters consistent with those in Section 8.4. 


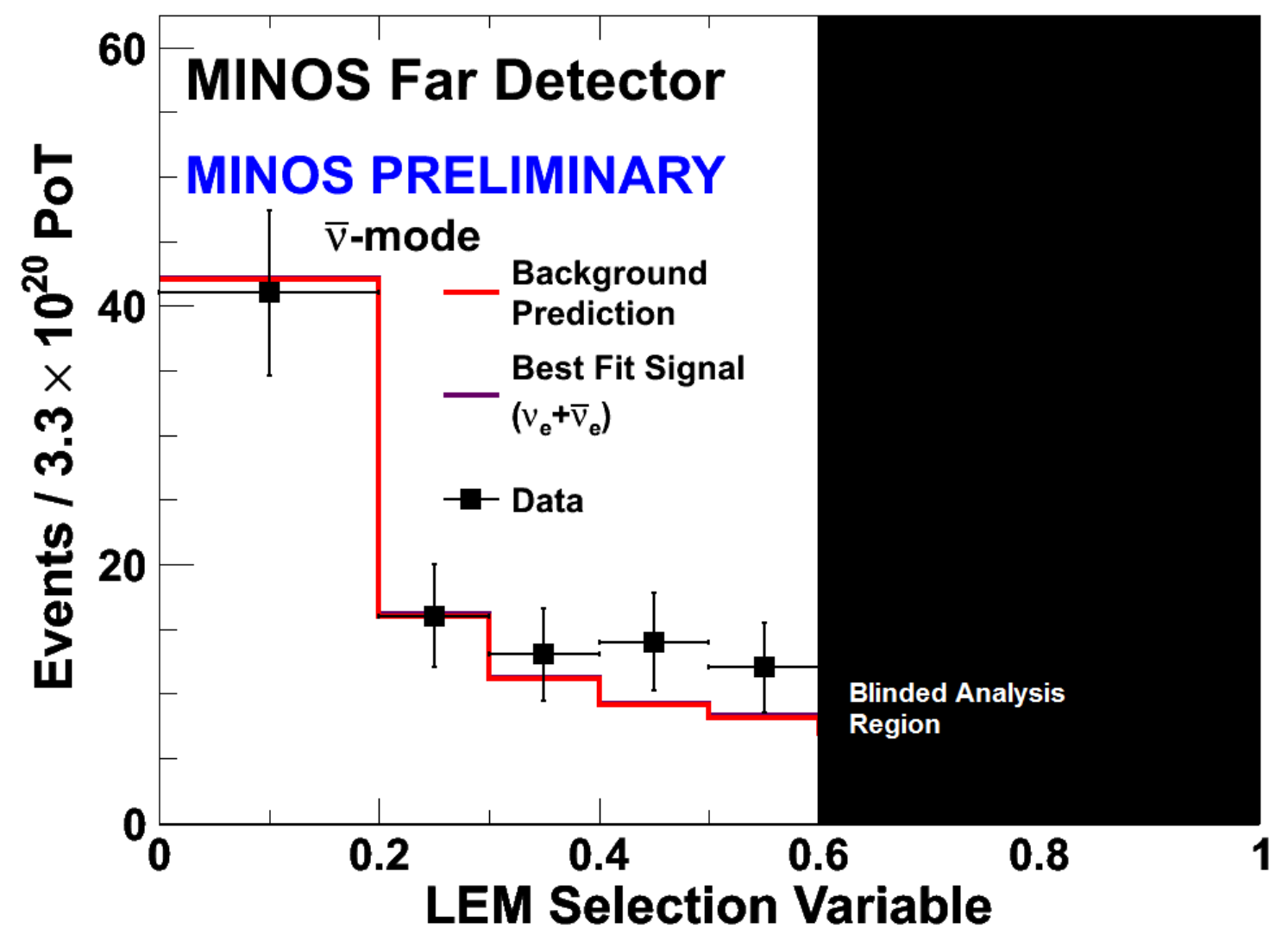

Figure 10.2: LEM PID distribution in the RHC Anti-PID sideband for the Far Detector prediction and data. Oscillation parameters consistent with those in Section 8.4.

Considering that $77 \%$ of the FHC data had already been unblinded in the $3^{\text {rd }}$ appearance analysis, an additional check, dubbed the Glass Box sideband, was added to validate the new FHC LEM PID algorithm. Defining this check as a sideband warrants some explanation. After all, the Glass Box does contain signal candidates of interest, a fact which does not really adhere to the conventional definition of a sideband. However, the aim of this step was to verify that the new PID algorithm did not produce an inexplicable discrepancy in the number of selected events when compared to the old PID. In other words, we wanted to confirm that both PIDs would yield similar event counts despite the changes in reconstruction, the development of a new algorithm, et cetera. In this regard, classifying the Glass Box as a sideband is legitimate, for we are comparing the numbers 
of events in the well-known runs of Period I before examining the blinded signal region in Period III.

One of the more interesting results of the Glass Box study was the observation of PID value migration. Using the $3^{\text {rd }}$ analysis result as a prediction, one would expect $109 \pm$ 10.4 Far Detector events in the LEM $>0.6$ region and $62 \pm 7.9$ Far Detector events with LEM $>0.7$. The results for the current analysis were 118 and 64, respectively, and while these quantities were both within one standard deviation, the differences were investigated on an event-by-event basis.

Between the $3^{\text {rd }}$ and $4^{\text {th }}$ appearance analyses, a total of 12 event migrations (10 out, 2 in) at the preselection cut level were observed. These were largely due to changes in the energy reconstruction that occurred after the $3^{\text {rd }}$ analysis which resulted in events with energies near 1 and $8 \mathrm{GeV}$ drifting across the preselection threshold. This particular discrepancy generated some concern amongst MINOS collaborators; however, all 12 of these events had LEM PIDs $<0.2$ and posed no threat to the validity of the signal region. Net migrations across the signal selection thresholds were expected at around the 5\% level due to variations associated with the retraining of the artificial neural network. An X-Y scatter plot, shown in Figure 10.3 , shows the $3^{\text {rd }}$ vs. $4^{\text {th }}$ PID distributions for the 539 common preselected events. In total, 2 events exited and 1 event entered the signal-rich LEM PID $>0.7$ region. 16 events left and 14 events entered the PID region used for the final fit. ${ }^{(62)}$

The net migration of events in the signal-selected PID regions when comparing the two FHC LEM PIDs met the group's expectations. Recall that the agreement in the total 
number of events in the signal-selected regions was better than $1 \sigma$. Investigating events in the Glass Box indicated that the new FHC LEM PID was behaving as expected, and the study showed that new PID returned an answer consistent with the previous analysis.

\section{3rd vs 4th Analysis LEM}

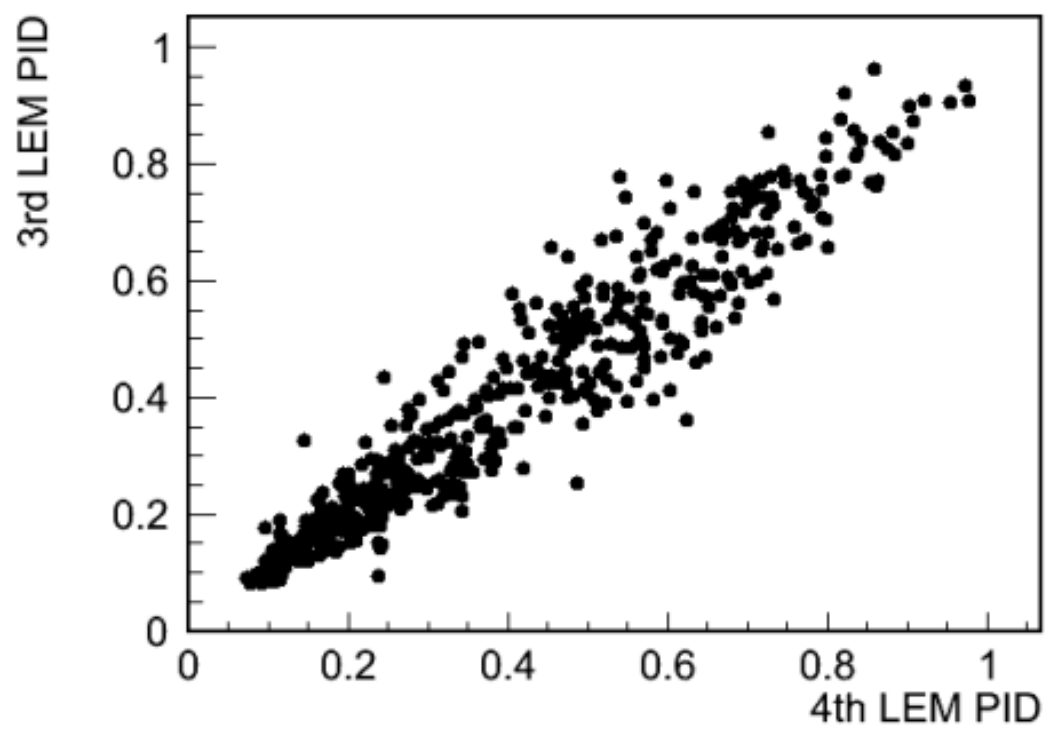

Figure 10.3: $3^{\text {rd }}$ vs. $4^{\text {th }}$ analysis LEM PID scatter plot using the 539 common events in the preselection samples. This plot was generated as part of the PID value migration study and shows a strong correlation between the old and new FHC LEM algorithms. Image from (62).

The results of the Anti-PID and Glass Box studies led to the unblinding of the complete MINOS data set. However, a third sideband was looked at retroactively for this thesis as well as an upcoming publication to be submitted to Physical Review D. The MRCC sideband consists of $v_{\mu} \mathrm{CC}$ events in which the muon track has been removed. The resulting sample contains NC-like events, as the track-removed event contains dominantly hadronic shower energy depositions. As these samples were created using well-defined $v_{\mu} \mathrm{CC}$ events, the MRCC sets have no signal events, a feature which permits access to all values of the LEM PID. The MRCC sideband allows us to assess if the data- 
MC discrepancy due to hadronic modeling exists equally in both detectors. A large difference in the discrepancies would indicate an unidentified difference between the Near and Far Detector.

Events in the MRCC samples are selected with a series of primary cuts that include a data quality assessment, a 1-8 GeV reconstructed energy cap (to facilitate direct comparison with the appearance analysis preselection sample), and two MRCC-specific cuts established for the $v_{\mu} \mathrm{CC}$ analysis. The first selects only events that occurred within the $\mathrm{CC}$ fiducial regions of the detectors; and the second requires that the event contains a well-reconstructed/fit track and has an original roCC PID value $>0.3 .{ }^{(63)}$ Interactions that pass these initial cuts compose the base-level MRCC samples. Additional appearance cuts (the standard appearance analysis fiducial and preselection) are applied as well. Monte Carlo samples are scaled to a total 1e19 POT, and the Near Detector data is scaled to match the POT associated with the Far Detector sample. Naively, the simplest way to investigate the handling of hadronic showers in the MRCC sideband would be to generate a Far Detector MRCC prediction using the Near Detector MRCC data and MRCC Monte Carlo. Since the MRCC sideband only contains events of one type, no Near Detector beam decomposition needs to be performed. The extrapolation is carried out according to the prescription defined in Expression (5.2), with one additional caveat.

An additional scaling factor is introduced for each bin in the PID distribution that reweights the prediction by the ratio of base-level data:predicted events. This ensures that, prior to the introduction of the $v_{e}$-related analysis cuts, there are an effective equal 
number of events populating the MRCC Prediction and Far Detector data samples. This scale factor accounts for a well-documented excess in the base-level MRCC sample, as described in (64). Simply put, the factor was introduced to correct an inherent discrepancy between the data and prediction that was completely unrelated to the $v_{e}$ analysis. Once applied, any differences observed between the Far Detector MRCC data and prediction could be attributed to the techniques and methods used in the appearance analyses. Historically, a better than $2 \sigma$ agreement has been sought.

Over the full range of the LEM PID, we expected 191.0 $\pm 13.8_{\text {stat }}$ events in the RHC Far Detector MRCC sample while 198 were observed, yielding a $0.51 \sigma$ discrepancy. In the LEM $>0.6$ region, $32.2 \pm 5.7_{\text {stat }}$ events were expected with 34 observed, yielding a $0.32 \sigma$ difference. In the FHC case, $808.7 \pm 28.4_{\text {stat }}$ events were expected in the full Far Detector PID distribution and 808 were observed. In the LEM $>0.6$ range, $188.6 \pm 13.7_{\text {stat }}$ events were expected with 191 observed, yielding a $0.18 \sigma$ difference. The LEM PID distributions for the RHC and FHC MRCC sidebands are shown in Figure 10.4. Errors presented are purely statistical. 


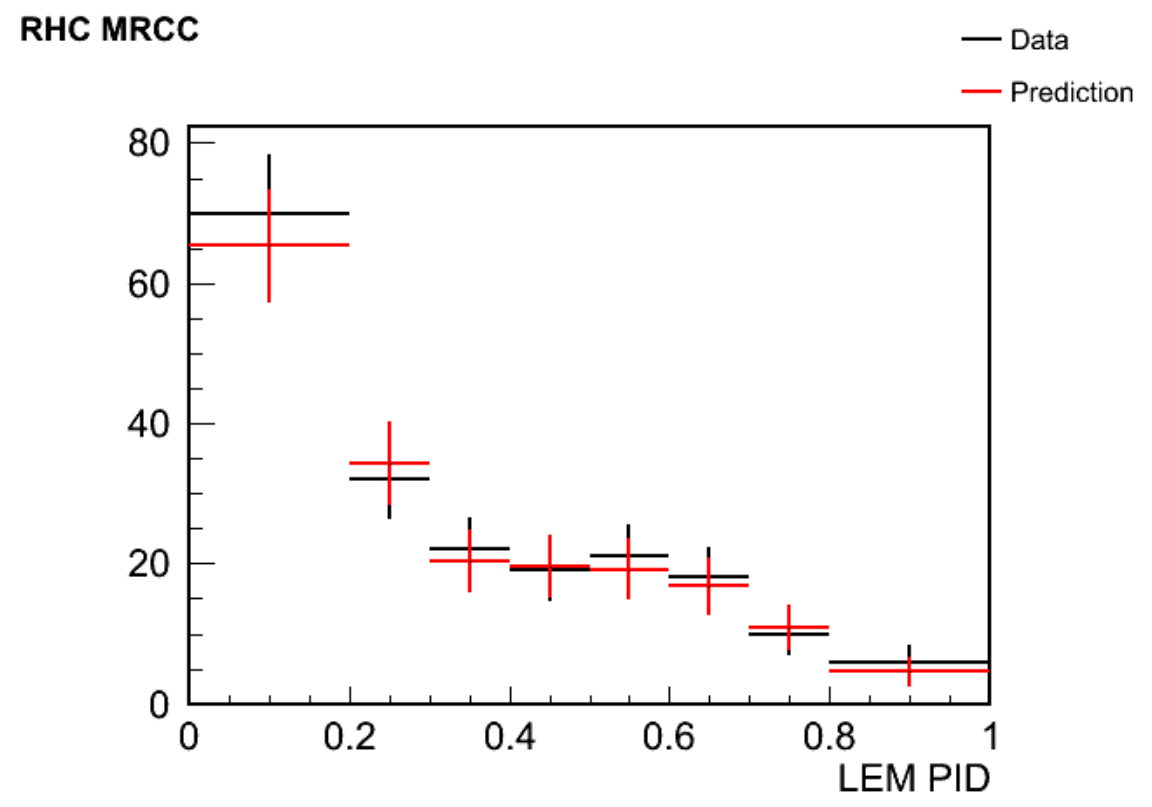

FHC MRCC

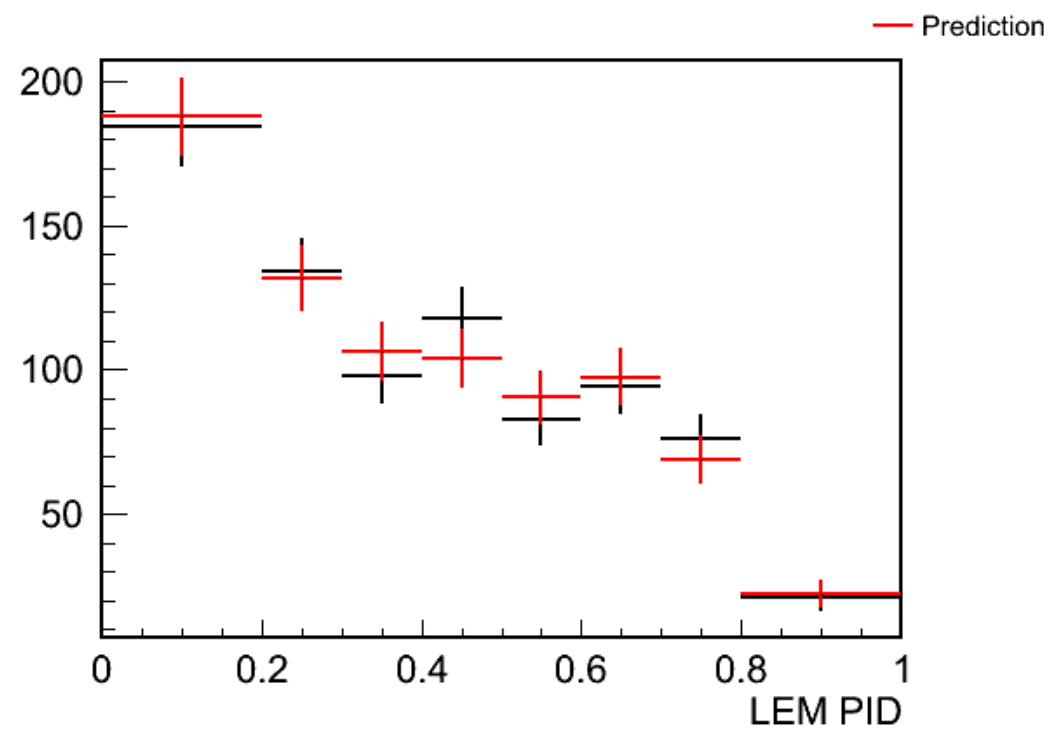

Figure 10.4: $R H C$ and FHC MRCC Sideband PID distributions. The black lines represent the Far Detector MRCC Data while the red lines are the Far Detector MRCC predictions. Only statistical errors are included. 


\subsection{Unblinded Data}

The sideband checks presented in the previous section yielded no evidence to suggest the presence of unexpected behavior stemming from our analysis procedures and extrapolation techniques. Thus, the LEM $>0.6$ regions of the $\mathrm{FHC}$ and $\mathrm{RHC}$ samples are unblinded and investigated. Before generating the Feldman-Cousins contours that define the $90 \%$ C.L. in $2 \sin ^{2}\left(\theta_{23}\right) \sin ^{2}\left(2 \theta_{13}\right)$ for all values of $\delta_{\mathrm{CP}}$ and both mass hierarchies, simple counting experiments were performed in the signal-rich, LEM $>0.7$ range. As mentioned near the end of Chapter 8 , we expected $69.1 \pm 8.3_{\text {stat }} \pm 4.3_{\text {syst }}$ background events in the FHC mode, and $10.5 \pm 3.2_{\text {stat }} \pm 0.6_{\text {syst }}$ background events in the RHC mode. 88 events were observed in the FHC sample, and 12 events were observed in the RHC sample. While excesses in the signal region have been seen, neither of these event counts provided definitive, statistically significant evidence that $v_{\mu} \rightarrow v_{\mathrm{e}}$ or $\bar{v}_{\mu} \rightarrow \bar{v}_{\mathrm{e}}$ oscillations occurred in the MINOS data. The full Far Detector PID distributions for the FHC and RHC modes are shown in Figure 10.5.

The numbers of signal candidates in the LEM $>0.7$ regions do not provide a clear picture of the oscillation modes in question. At this level, we are unable to distinguish our observed event counts from both the null oscillation background prediction and the signal expected when $\theta_{13}$ is near the value suggested by reactor neutrino experiments. To glean more information from the data, we rely upon our fitting technique to place limits on the relevant oscillation parameters. 

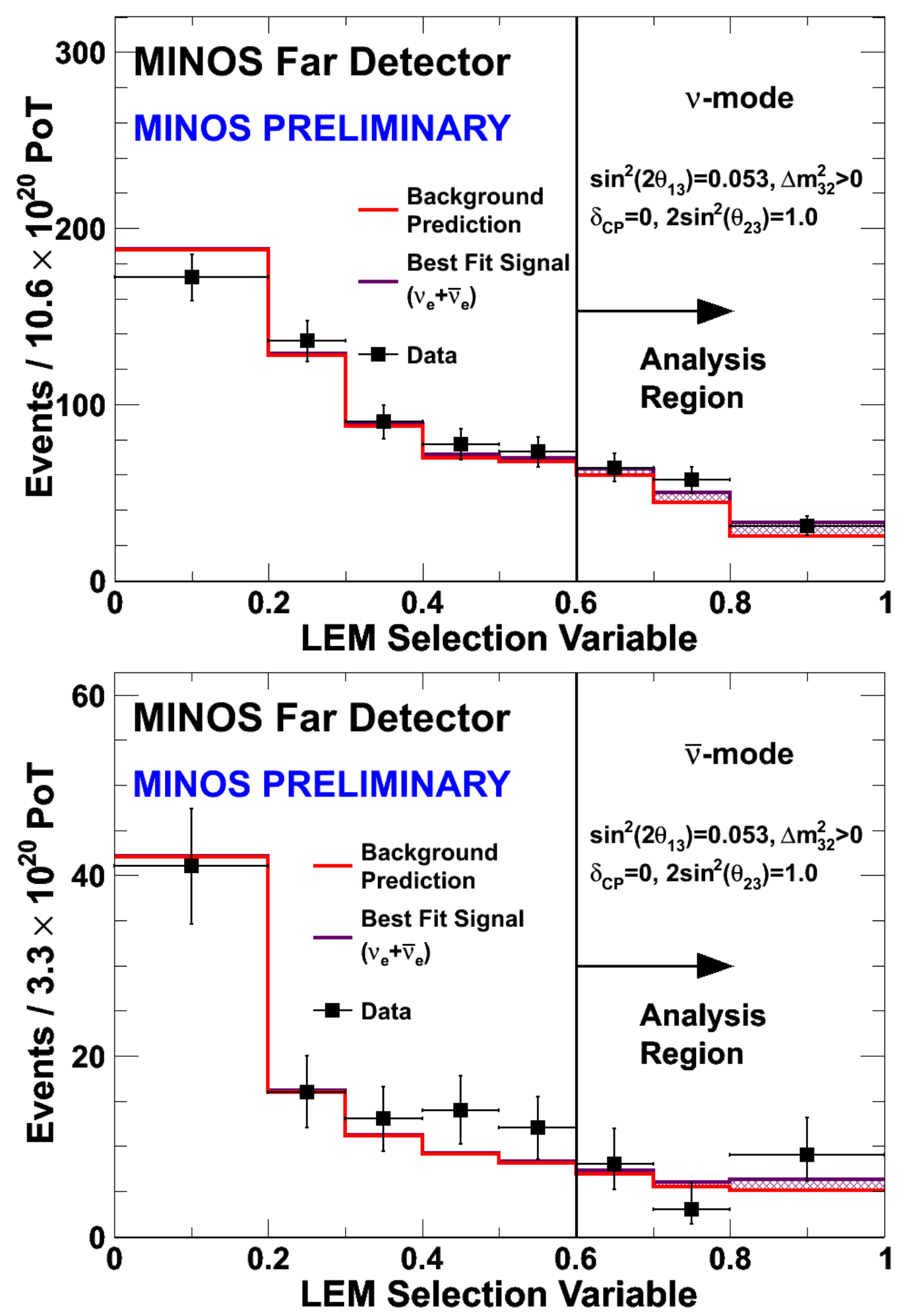

Figure 10.5: Far Detector PID distributions for FHC (top) and RHC (bottom) modes. The full unblinded region ( $L E M>0.6)$ is used for the fit, while the $L E M>0.7$ region was used for the event counting experiments. 


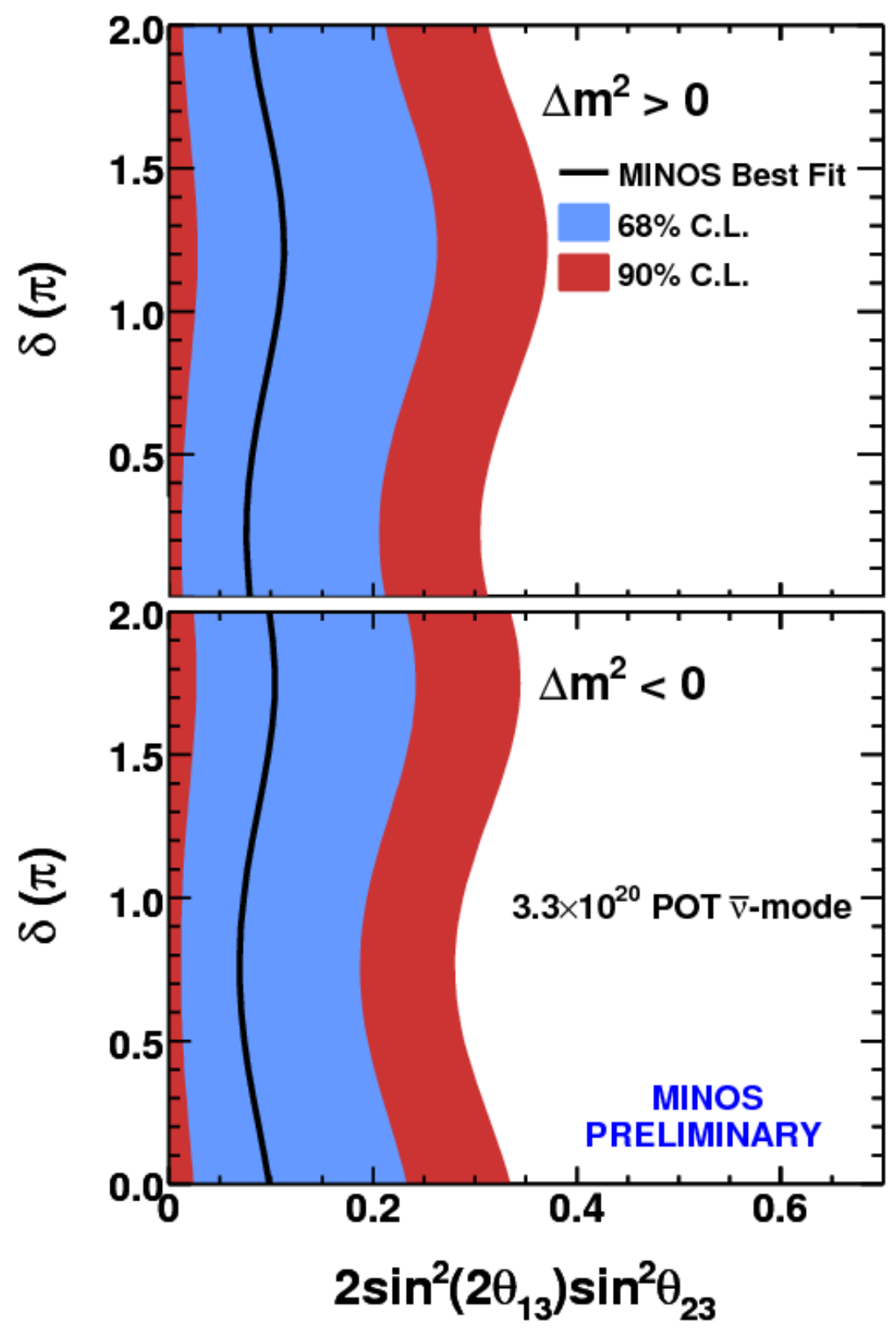

Figure 10.6: $R H C$ only Feldman-Cousins contours showing the allowed ranges of $2 \sin ^{2}\left(\theta_{23}\right) \sin ^{2}\left(2 \theta_{13}\right)$ for normal (top) and inverted (bottom) mass hierarchies. At $\delta_{C P}=0$, with normal (inverted) hierarchy, the best fit located at 0.079 (0.098) with an allowed $90 \%$ C.L. range of $2 \sin ^{2}\left(\theta_{23}\right) \sin ^{2}\left(2 \theta_{13}\right)<0.31(0.34)$. 


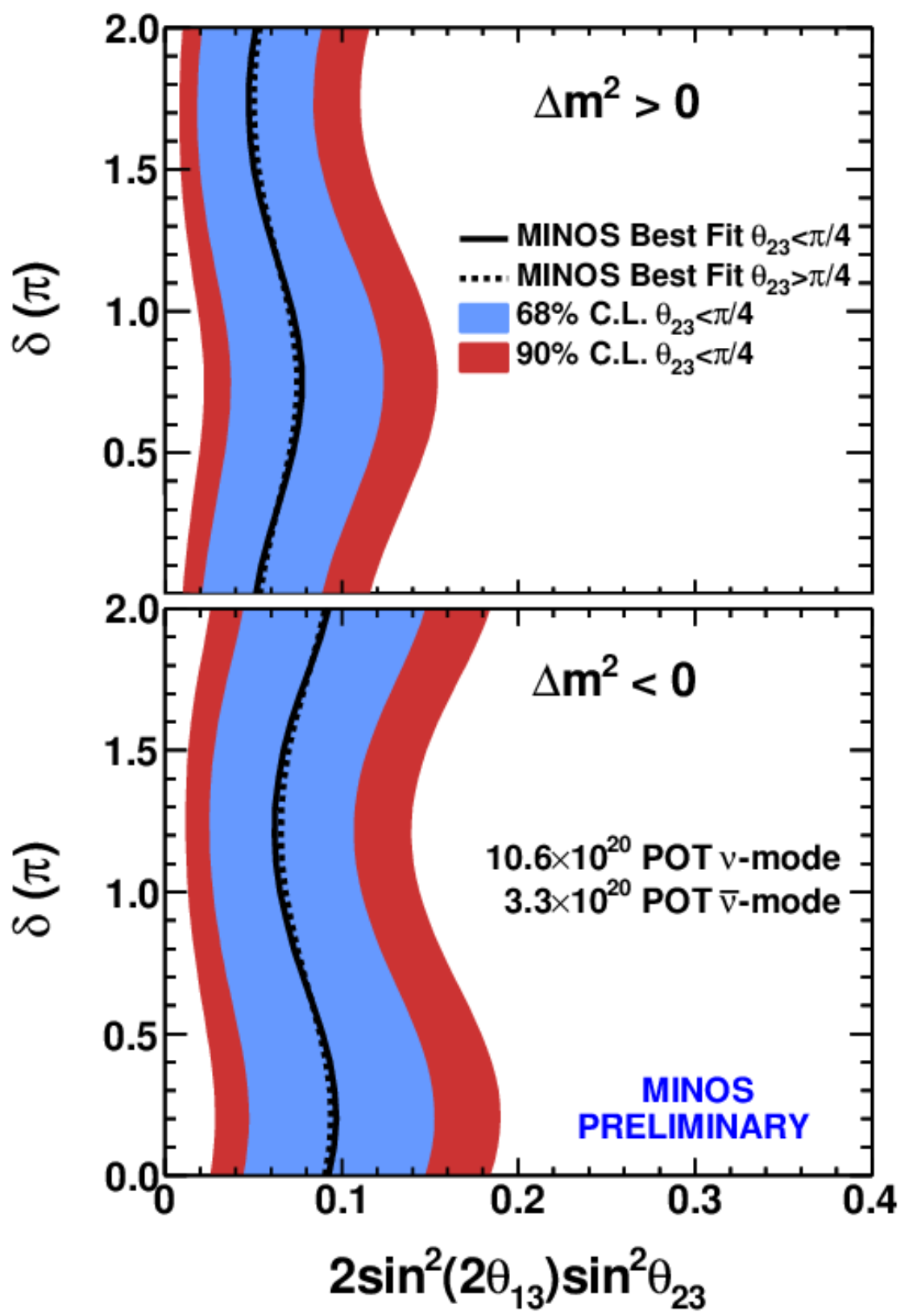

Figure 10.7: $F H C+R H C$ Feldman-Cousins contours showing the allowed ranges of $2 \sin ^{2}\left(\theta_{23}\right) \sin ^{2}\left(2 \theta_{13}\right)$ for normal (top) and inverted (bottom) mass hierarchies. At $\delta_{C P}=0$, with normal (inverted) hierarchy, the best fit located at 0.051 (0.093) with an allowed $90 \%$ C.L. range of $0.01(0.03)<2 \sin ^{2}\left(\theta_{23}\right) \sin ^{2}\left(2 \theta_{13}\right)<0.12(0.18)$. 
Figures 10.6 and 10.7 show the Feldman-Cousins contours for the RHC-only and FHC + RHC fits. Both the $68 \%$ and $90 \%$ C.L. bands are presented. Note that Figure 10.7 contains two best fit contours depending on whether the value of $\theta_{23}$ is in the first or second octant. In mid-2012, MINOS presented evidence suggesting that $\theta_{23}$ was not $\pi / 4$.

${ }^{(65)}$ In the $v_{\mu} \mathrm{CC}$ analysis, the contours are parameterized in terms of $\left|\Delta m^{2}\right|$ and $\sin ^{2} 2 \theta$. The statement on the value of $\theta_{23}$ is reflected in the $v_{\mu} \mathrm{CC}$ (shown in Figure 10.8) result by a best fit point that is significantly removed from the maximum possible value of $\sin ^{2} 2 \theta$. As the dominant contribution of $\theta_{23}$ in the appearance probability function is $\sin ^{2} \theta_{23}$ and not $\sin ^{2} 2 \theta_{23}$, the best fit point from the $v_{\mu} \mathrm{CC}$ analysis yields an optimal $\theta_{23}$ value on each side of $\pi / 4$ physical boundary. Hence, two Feldman-Cousins best fit contours were generated in which $\theta_{23}$ was allowed to vary over the $1 \sigma$ range in each octant. This octant separation of allowed ranges of $\theta_{23}$ was continued in the $\delta_{\mathrm{CP}}-$ hierarchy investigation.

For the RHC-only sample, at $\delta_{\mathrm{CP}}=0$ with normal (inverted) mass hierarchy, the best fit point in $2 \sin ^{2}\left(\theta_{23}\right) \sin ^{2}\left(2 \theta_{13}\right)$ is located at $0.079(0.098)$ with a $90 \%$ C.L. range of $2 \sin ^{2}\left(\theta_{23}\right) \sin ^{2}\left(2 \theta_{13}\right)<0.31(0.34)$. The null hypothesis is excluded at the $80 \%$ confidence level. For the complete MINOS data set, at $\delta_{\mathrm{CP}}=0$ with normal (inverted) mass hierarchy, the best fit point in $2 \sin ^{2}\left(\theta_{23}\right) \sin ^{2}\left(2 \theta_{13}\right)$ is located at $0.051(0.093)$ with a $90 \%$ C.L. range of $0.01(0.03)<2 \sin ^{2}\left(\theta_{23}\right) \sin ^{2}\left(2 \theta_{13}\right)<0.12(0.18)$. The null hypothesis is excluded at the $96 \%$ C.L.

\section{$10.3 \delta_{\mathrm{CP}}$-Hierarchy Investigation}

Two of the primary focal points for the next generation neutrino physics experiments 
include the determination of the mass hierarchy and the identification of $\delta_{\mathrm{CP}}$. Prior to the determination of $\theta_{13}$, the extent to which MINOS could evaluate these parameters was minimal at best. However, the 2012 reactor experiments have facilitated an investigation using the MINOS data by providing the $0.0982 \pm 0.0131$ constraint on the value of $\sin ^{2}\left(2 \theta_{13}\right) .{ }^{(1)(2)(3)}-2 \ln \mathcal{L}$ contours are produced as a function of $\delta_{\mathrm{CP}}$ for four different oscillation parameter possibilities that cover the mass hierarchy and $\theta_{23}$ octant combinations. The method with which the contours are generated works as follows:

1. An initial 1D scan is performed over $\delta_{\mathrm{CP}}$ with other oscillation parameters set to the standards listed in $8.4 . \delta_{\mathrm{CP}}$ is incremented by $0.01 \pi$ over the fill $2 \pi$ range, the prediction is extrapolated, and $-2 \ln \mathcal{L}$ is calculated for each point. The point of $\delta_{\mathrm{CP}}$ at which $-2 \ln \mathcal{L}$ is minimized is fed as the central point for the second step in the process.

2. A 40,000 point, $4 \mathrm{D}$ grid search is performed over $\delta_{\mathrm{CP}}, \theta_{13}, \theta_{23}$, and $\Delta m_{32}^{2}$ for each of the four combinations. $\delta_{\mathrm{CP}}$ is incremented over a 0.1 radian window centered about the best fit point from Step 1. The profile limits of the other three parameters are set by the choice in hierarchy, $\theta_{23}$ octant, the experimental best fit values, and the $\pm 1 \sigma$ intervals for those results. The predictions are made for each $4 \mathrm{D}$ grid point and the global minimum in $-2 \ln \mathcal{L}$ for each contour is found. Note that penalty terms following the functional form of $\left(x_{i}-\mu\right)^{2} / \sigma^{2}$ were applied for $\theta_{13}, \theta_{23}$, and $\Delta m_{32}^{2}$ variables (where $x_{i}$ is oscillation parameter $x$ at the $i^{\text {th }}$ increment, $\mu$ is the mean value of the parameter, and $\sigma$ is the associated uncertainty) during the grid search to enforce the Gaussian distributions of the experimental priors. The parameter values at the best fit point are then fed as extrapolation inputs for Step 3. 
3. A final sweep over $\delta_{\mathrm{CP}}$ is performed. The extrapolation is done with the oscillation parameters $\theta_{13}, \theta_{23}$, and $\Delta m_{32}^{2}$ set to the values identified by the $4 \mathrm{D}$ search in Step 2 . $-2 \ln \mathcal{L}$ is plotted as a function of $\delta_{\mathrm{CP}}$ for the four possible combinations of neutrino mass hierarchy and $\theta_{23}$ octant.

4. The absolute minimum $-2 \ln \mathcal{L}$ value is subtracted to produce $-2 \Delta \ln \mathcal{L}$ contours. In this picture, the point at which $-2 \Delta \ln \mathcal{L}=0$ indicates the mass hierarchy, $\theta_{23}$ octant, and value of $\delta_{\mathrm{CP}}$ which yields the best match to the observed data. The significance of exclusion for regions of the phase space are thus determined by value of $-2 \Delta \ln \mathcal{L}$ at the points in question.

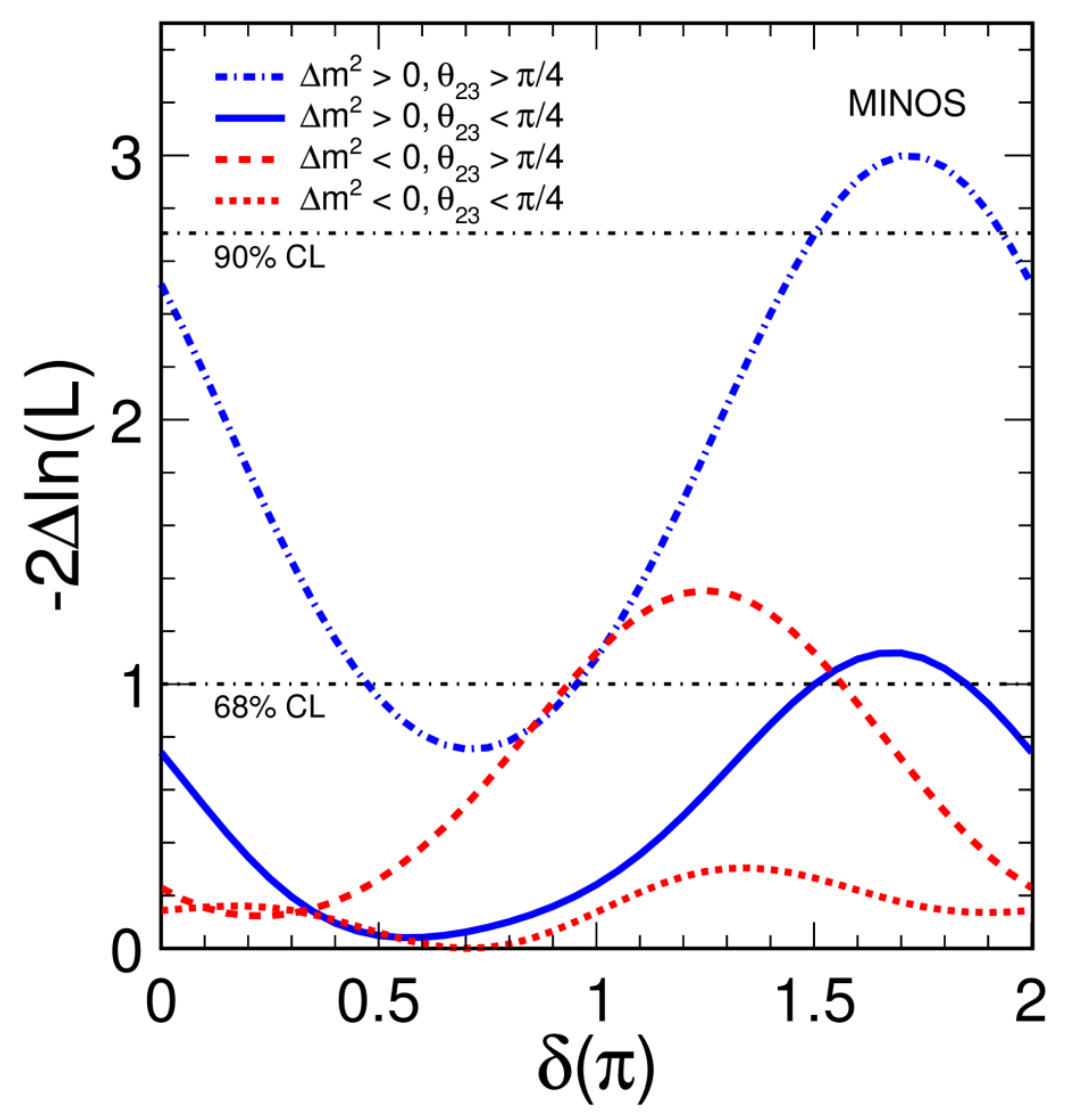

Figure 10.8: $-2 \Delta \ln \mathcal{L} v s . \delta_{C P}$ contours for the four possible combinations of mass hierarchy and $\theta_{23}$ octant using the complete MINOS data set. 
The results shown in Figure 10.8 indicate that our data show a slight preference for the inverted mass hierarchy with $\theta_{23}<\pi / 4$. The preferred IH, Octant 1 minimum bests the $\mathrm{NH}$, Octant 1 minimum by 0.04 units of $-2 \ln \mathcal{L}$. The MINOS data disfavor $31 \%(5 \%)$ of the parameter space at the $68 \%$ C.L. $\left(90 \%\right.$ C.L.). No definite conclusions regarding $\delta_{\mathrm{CP}}$ or hierarchy were reached by analyzing the complete MINOS data.

Ultimately, this study yields two promising pieces of information. First, the technique needed to perform this analysis was developed and implemented. It made use of the most recent oscillation parameter results, was relatively simple to execute compared to the Feldman-Cousins prescription, extended the MINOS physics reach well beyond the original scope outlined by the disappearance analysis, and set a possible standard for future experiments. In addition, while nothing definitive can be said about $\delta_{\mathrm{CP}}$ based solely upon this investigation, our data are beginning to constrain the values of the $\mathrm{CP}$ violating phase, and they favor non-integer values of $\delta_{\mathrm{CP}} / \pi$ in all four contours. This last point is a subtle hint that perhaps more interesting physics is occurring in the lepton sector, and it lays the case for supporting future neutrino experiments capable of constructing a clearer picture.

\subsection{Results Summary}

For the first time, simultaneous appearance analyses were conducted over the entire MINOS neutrino and antineutrino data sets. Using the Library Event Matching signal selection technique, excesses of electron neutrinos and antineutrinos were observed in the data. These surpluses yielded results consistent with those presented by the reactor experiment community. 
Referencing the joint fit, our data exclude the $\theta_{13}=0$ hypothesis at the $96 \%$ confidence level, marking the first time the official MINOS exclusion has exceeded $90 \%$. Once more, at $\delta_{\mathrm{CP}}=0$ with normal (inverted) mass hierarchy, the best fit point in $2 \sin ^{2}\left(\theta_{23}\right) \sin ^{2}\left(2 \theta_{13}\right)$ is located at $0.051(0.093)$ with a $90 \%$ C.L. range of $0.01(0.03)<2 \sin ^{2}\left(\theta_{23}\right) \sin ^{2}\left(2 \theta_{13}\right)<0.12(0.18)$. The $\delta_{\mathrm{CP}}$-hierarchy investigation was also a first for the MINOS collaboration. Relying upon the reactor experiment results as well as the long-baseline nature of the MINOS experiment, we implemented a 4D grid search fitting technique to provide insights into the limits on $\delta_{\mathrm{CP}}$ when specific mass hierarchy and $\theta_{23}$ octant restrictions were applied. Despite the absence of a definitive result, this procedure surpassed the original analysis goals and - more importantly - highlighted a technique that could perhaps be adopted by the next generations of neutrino experiments. Considering that this will be the last appearance search conducted by MINOS, the results and legacy of the $\delta_{\mathrm{CP}}$-hierarchy investigation serve as an excellent segue in to the next generation experiments and the corresponding unknowns of neutrino physics lurking over the horizon. 


\section{Chapter 11}

\section{Welcome to the World of Tomorrow}

\subsection{Where the Field Stands}

A year ago, much of the talk amongst experimental neutrino physicists was the ongoing search for $\theta_{13}$. The parameter was the last mixing angle demanding a measurement, and it is the gateway to studying CP-violation in the lepton sector. Numerous experiments, in addition to MINOS, sought to improve their sensitivities to the relevant oscillation modes as well as their analysis techniques.

$\mathrm{T} 2 \mathrm{~K}$, a long baseline neutrino experiment in Japan, released results at the same time as the $3^{\text {rd }}$ MINOS appearance analysis, which provided further hints of a nonzero $\theta_{13}{ }^{(66)}$ The overlaid MINOS+T2K contours, shown in Figure 11.1, generated hopes that a combined analysis between the two experiments would have culminated with a more definitive answer (MINOS reported tighter constraints whereas T2K's lower 90\% C.L. was at larger value of $\theta_{13}$ than the MINOS result).

The NuMI Off-Axis $v_{\mathrm{e}}$ Appearance (NOvA) accelerator-based experiment emerged as the heir-apparent to MINOS as a candidate to push the $\theta_{13}$ question. The experiment, using liquid scintillator and PVC, offered better sensitivity than the MINOS detectors. ${ }^{(67)}$ Reactor experiments, however, completely changed the scope of the field with the release of a trio of 2012 results. In the span of a few months, $\theta_{13}$ went from an unknown parameter to one of the most precisely known quantities in neutrino physics. Double Chooz, based in France, released the first of the three results using 101 days of data from their Far Detector, which is located 1050 meters from the two 4.25 GW Chooz 
Reactors. Double Chooz excluded the $\theta_{13}=0$ hypothesis at the $94.6 \%$ C.L. using shape and rate information from the prompt positron spectrum. ${ }^{(3)}$

The second result came from Daya Bay, which used six liquid-scintillator detectors located at various distances along a flux-weighted baseline. With data collected over 55 days, Daya Bay measured $\sin ^{2} 2 \theta_{13}=0.092 \pm 0.016_{\text {stat }} \pm 0.005_{\text {syst }}$ and ruled out the $\theta_{13}=0$ hypothesis at 5.2 ${ }^{(1)}$ The third $\theta_{13}$ measurement came from RENO, which used two detectors placed along a flux-weighted baseline near six $2.8 \mathrm{GW}$ nuclear reactors in South Korea. Using a rate-only analysis on 229 days of data, RENO measured $\sin ^{2} 2 \theta_{13}=$ $0.113 \pm 0.013_{\text {stat }} \pm 0.019_{\text {syst }}$ and excluded the $\theta_{13}=0$ hypothesis at $4.9 \sigma{ }^{(2)}$

Interestingly, the updated MINOS analysis reported in this thesis is competitive with the Double Chooz null hypothesis exclusion. As mentioned in Section 10.3, these measurements were invaluable requisites for performing the MINOS $\delta_{\mathrm{CP}}$-hierarchy study. The 2012 reactor results, along with previous (2011 and earlier) MINOS, T2K, and Chooz reports, are shown in Figure 11.2. 

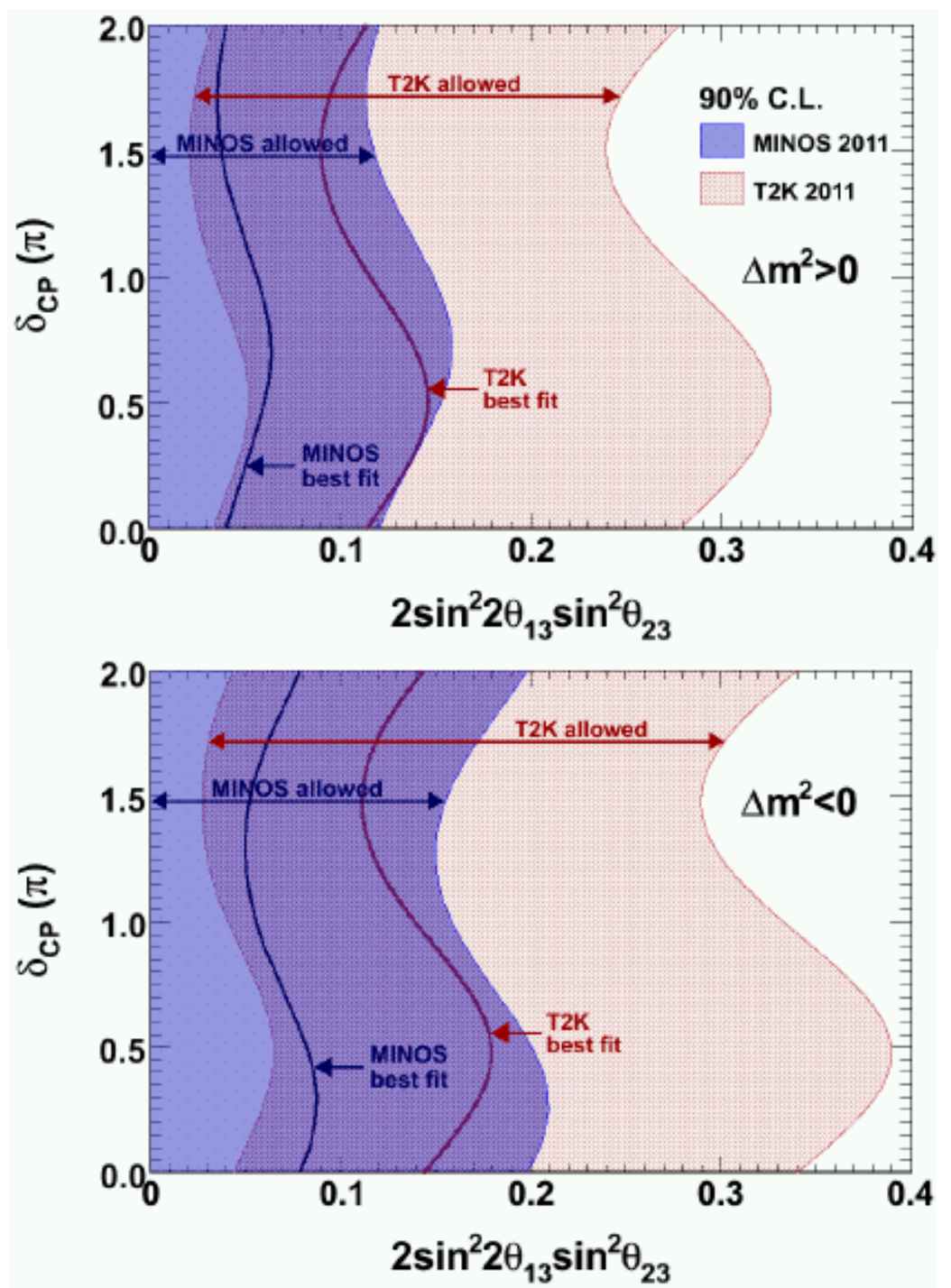

Figure 11.1: Overlaid 90\% C.L. regions from the MINOS 2011 and T2K 2011 results in terms of $\delta_{C P}$ and $2 \sin ^{2}\left(\theta_{23}\right) \sin ^{2}\left(2 \theta_{13}\right)$. Results from (31) and (66). 

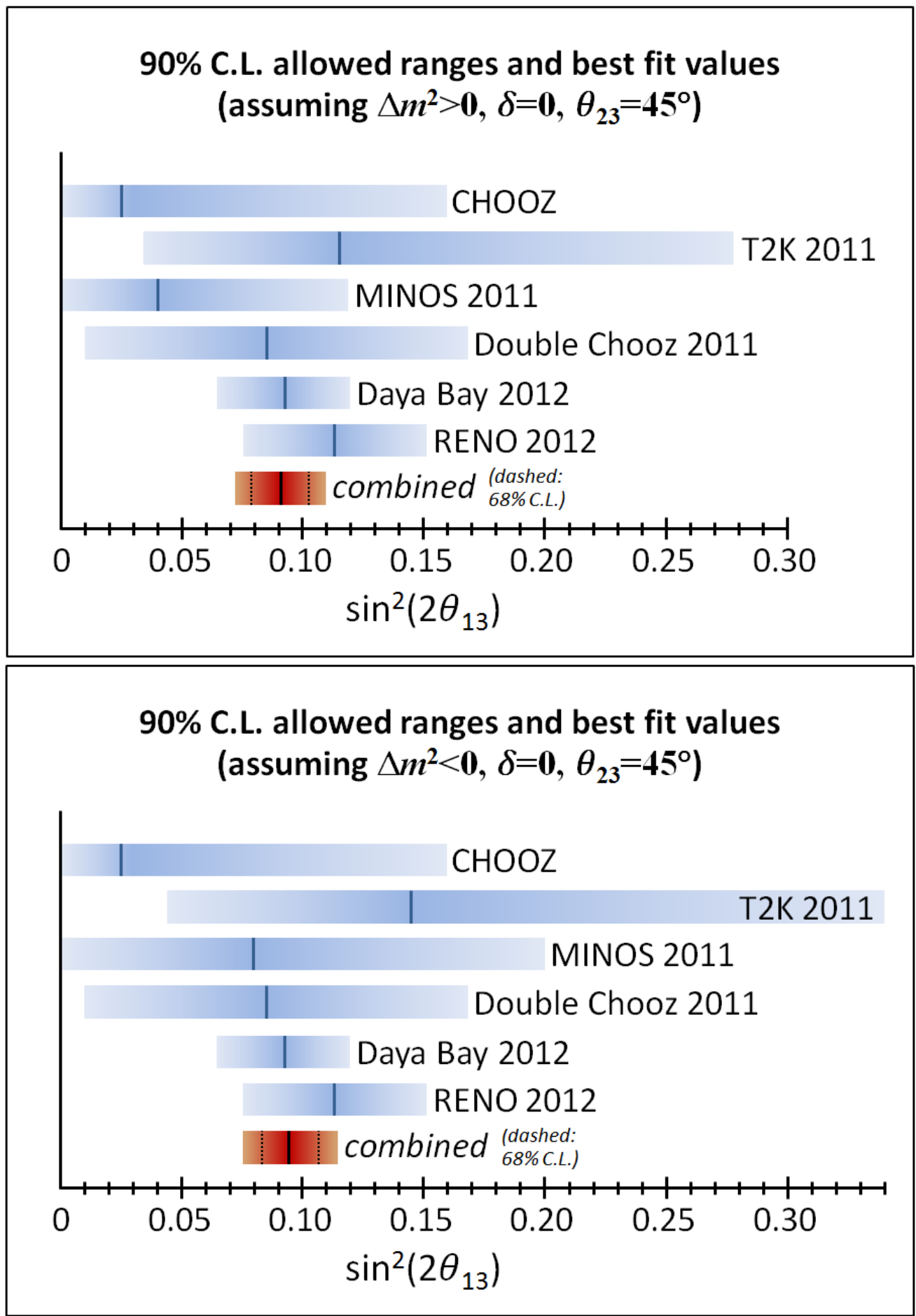

Figure 11.2: Overview of $\sin ^{2}\left(2 \theta_{13}\right)$ measurements from various neutrino experiments for normal (top) and inverted (bottom) mass hierarchies. The bands indicate the $90 \%$ confidence levels for each measurement. The dashed line in the combined band indicates the $68 \%$ C.L. Image from (68). 
The measurement of $\theta_{13}$ undoubtedly changed the landscape of neutrino physics.

However, several important questions pertaining to neutrinos still remain to be solved.

1. Is the neutrino mass hierarchy normal or inverted?

2. Is CP-violation present in the lepton sector? What is $\delta_{\mathrm{CP}}$ ?

3. MINOS results have hinted that atmospheric neutrino mixing is non-maximal. In which octant does $\theta_{23}$ reside?

4. What is the neutrino mass?

5. Are neutrinos Dirac or Majorana particles? In other words, are neutrinos and antineutrinos identical or not?

\subsection{Where the Field is Going}

The questions related to hierarchy and CP-violation will be probed by the NOvA experiment, which uses its long, $810 \mathrm{~km}$ baseline to probe the mass hierarchy via the MSW effect. ${ }^{(67)}$ Much like MINOS, NOvA will have the ability to run in a neutrino and antineutrino-enriched mode based on the polarity of the NuMI horn current. Given the better energy resolution in the NOvA detectors, the experiment will yield tighter constraints on the value of $\sin ^{2}\left(2 \theta_{23}\right)$ that could resolve the lingering octant issue as well. The Far Detector is currently under construction in Ash River, MN, and data from the first block (roughly $1 / 28^{\text {th }}$ of the full detector) is expected to be taken starting in February 2013 with beam data coming that April. ${ }^{(69)}$

The answer to the question of neutrino mass resides firmly outside the realm covered by the neutrino oscillation experiments discussed thus far. The KATRIN tritium beta decay experiment aims to ascertain the electron antineutrino mass with sub-eV sensitivity using 
precision measurements of the electron energy spectrum. ${ }^{(70)}$ With regards to the Dirac vs. Majorana question, double beta decay experiments serve as the focal point. If neutrinos are Majorana in nature, then it would be possible for the two neutrinos released in double beta decay to annihilate, producing a neutrinoless double beta decay signature. An observation of such an event (in which an isotope decays through the emission of only two electrons) would yield substantial evidence to show neutrinos were Majorana particles. Experiments such as $\mathrm{COBRA}^{(71)}, \mathrm{EXO}^{(72)}, \operatorname{GERDA}^{(73)}$, Majorana ${ }^{(74)}$, and NEXT $^{(75)}$ would be capable of making such a discovery.

\subsection{Doing It Differently}

In addition to the physics questions that remain unanswered in neutrino physics, we can always ask how to improve the reach of our experiments. While new analysis techniques can provide some solutions, new detector technologies can provide impressive gains. Liquid Argon Time Projection Chambers (LAr-TPC) represent one such technological leap, and they have already impacted neutrino physics. These detectors offer incredible spatial resolution (on mm order), especially when compared to resolution of the MINOS

experiment. Experiments such as ArgoNeuT ${ }^{(76)}$, ICARUS ${ }^{(77)}$, and MicroBooNe ${ }^{(78)}$ have all demonstrated the excellent performance of LAr-TPCs. LBNE (Long Baseline Neutrino Experiment) decided to use liquid argon TPCs after an extensive proposal period. While the experiment is currently undergoing restructuring, its use of the new detector technology would facilitate high-precision measurements. ${ }^{(79)}$

\subsection{Final Thoughts}

Regardless of the choice in adopted detector technology, all of the experiments 
mentioned in this chapter, as well as others, could unveil the answers to the questions outlined in Section 11.1. I eagerly await those solutions and hope that the research I do in the future - in addition to the research presented in this thesis - helps put the pieces of the neutrino puzzle together. 


\section{References}

1. Observations of Electron-Antineutrino Disappearance at Daya Bay. F.P. An et al. (Daya Bay). s.1. : Phys. Rev. Lett. 108:121803, 2012.

2. Observation of Reactor Electron Antineutrino Disappearance in the RENO Experiment. J.K. Ahn et al. (RENO). s.1. : Phys. Rev. Lett. 108:191802, 2012. 3. Indication of Reactor Electron Antineutrino Disappearance in the Double Chooz Experiment. Y. Abe et al. (Double Chooz). s.1. : Phys. Rev. Lett. 108:131801, 2012. 4. Detection of the Free Neutrino: a Confirmation. Cowan et al. s.1. : Science 124:3212.103, 1956.

5. Observation of the High-Energy Neutrino Reactions and the Existence of Two Kinds of Neutrinos. L.M. Lederman et al. s.1. : Phys. Rev. Lett. 9:36-44, 1962.

6. Observation of Tau Neutrino Interactions. DONuT Collaboration. s.1. : Phys. Lett. B, 504:218-224, 2001.

7. Inverse Beta Processes and Nonconservation of Leption Charge. Pontecorvo, B. 1957, JETP - Letters to the Editor.

8. Maki, Nakagawa and Sakata. Remarks on the Unified Model of Elementary

Particles. Progress of Theoretical Physics. November 1962.

9. Davis, R. et al. Search for Neutrinos from the Sun. Phys. Rev. Lett. 20:1205-1209.

May 1968.

10. Evidence for oscillation of atmospheric neutrinos. Super-Kamiokande

Collaboration. s.1. : Phys. Rev. Lett. 81:1562-1567, 1998.

11. SNO. Measurement of charged current interactions produced by 8B solar neutrinos at the Sudbury Neutrino Observatory. s.1. : Phys. Rev. Lett. 87:071301, 2001.

12. A. Höcker et al. . s.1. : Annu. Rev. Nucl. Part. Sci. 56:501-67, 2006.

13. Leptogenesis. S. Davidson et al. s.1. : Phys. Rept., 466:105-177, 2008.

14. Two Questions about Neutrinos. Kayser, B. s.1. : arXiv:1012.4469v1, 2010.

15. J. Beringer et al. (Particle Data Group). s.1. : Phys. Rev. D, 86:010001, 2012.

16. Five-Year Wilkinson Mincrowave Anisotropy Probe (WMAP) Observations:

Cosmological Interpreation. E. Komatsu et al. s.1. : Astrophys. J. Suppl. 180:330-376, 2009.

17. R. Patterson. New Results for $v \mu \rightarrow$ ve Oscillations in MINOS. s.1. : FNAL, 2010.

18. Series Expansions for Three-Flavor Neutrino Oscillation Probabilities in Matter.

E.K. Akhmedov et al. s.1. : Journal of High Energy Physics, 078, 2004.

19. Adamson, P. MINOS Plots. [Online]

http://adwww.fnal.gov/userb/pic/capture/www/minos-plots.html.

20. J. Hylen et al. NuMI Technical Design Handbook. [Online] http://www-

numi.fnal.gov/numwork/tdh/tdh_index.html.

21. A study of muon neutrino disappearance using the Fermilab Main Injector neutrino beam. P. Adamson et al. s.1. : Phys. Rev. D, 77:072002, 2008.

22. The magnetized steel and scintillator calorimeters of the MINOS experiment. MINOS

Collaboration. 2008, Nucl. Instr. and Meth. in Phys. Res. A. 
23. Schreckenberger, A.P. Electron Antineutrino Appearance in MINOS. s.1. : MINOS docdb-8375, 2011.

24. Orchanian, M. Electron Neutrino Apperance in the MINOS Experiment. s.1. : Caltech, 2012.

25. The magnetized steel and scintillator calorimeters of the MINOS experiment. D.G.

Michael et al. s.1. : Nucl. Instr. and Meth. A, 596:190-228, 2008.

26. Ochoa, J.P. A Search for Muon Neutrino to Electron Neutrino Oscillations in the MINOS Experiment. s.1. : Caltech, 2009.

27. R. Toner and A.P. Schreckenberger. An Updated Search for Electron Neutrino and Antineutrino Appereance in MINOS. s.1. : Neutrino 2012 \& Fermilab Users' Meeting, 2012.

28. MINOS Collaboration. Calibration Position Paper for Pre-Shutdown Data. 2008. MINOS-docdb 3941.

29. Electromagnetic Interactions in the MINOS Detectors. Vahle, P.L. s.1. : University of Texas at Austin, 2005.

30. Hadronic Interactions in the MINOS Detectors. Kordosky, M.A. s.1. : University of Texas at Austin, 2004.

31. P. Adamson et al. Improved search for muon-neutrino to electron-neutrino oscillations in MINOS. s.1. : arXiv:1108.0015v1, 2011.

32. MINOS Collaboration. MINOS Results. [Online] 2010, 2011. http://wwwnumi.fnal.gov/MinosResults/index.html.

33. Measurement of the neutrino mass splitting and flavor mixing by MINOS. P.

Adamson et al. s.1. : Phys. Rev. Lett. 106:181801, 2011.

34. Huang, X. Nue Calibration. s.1. : MINOS docdb-6702, 2011.

35. Schreckenberger, A.P. Nuebar Energy Scale Calibration for Dogwood3/5

Reconstruction. s.1. : MINOS docdb-8109, 2011.

36. New constraints on muon-neutrino to electron-neutrino transitions in MINOS. $\mathbf{P}$.

Adamson et al. s.1. : Phys. Rev. D, 82:051102, 2010.

37. Coelho, J.A.B. Near Detector Beam Decomposition Multi-Beam Method. s.1. : MINOS docdb-7986, 2011.

38. Schreckenberger, A.P. Nuebar Update Document. s.1. : MINOS docdb-8591, 2012.

39. MINOS Nue Group. RHC Executive Summary. s.1. : MINOS docdb-9031, 2012.

40. - FHC + RHC Executive Summary. s.1. : MINOS docdb-9097, 2012.

41. Hylen, J. Target location along beam-line; summary of entire MINOS run. s.1. : MINOS docdb-9314, 2012.

42. Search for muon-neutrino to electron-neutrino transitions in MINOS. P. Adamson et al. s.1. : Phys. Rev. Lett. 103:261802, 2009.

43. Holin, A. MRCC correction method for the determination of nue BG. s.1. : MINOS docdb-4018, 2008.

44. Ochoa, J.P. The Monte Carlo Nearest Neighbors Selection. s.1. : MINOS docdb$5031,2008$.

45. T. Yang and G. Pawloski. Crosstalk Tuning. s.1. : MINOS docdb-5319, 2008.

46. Ochoa, J.P. The status of the new MCNN. s.1. : MINOS docdb-4888, 2008.

47. Toolkit for multivariate data analysis. A. Hocker et al. s.l. : PoS, ACAT:040, 2007. 
48. Toner, R. Measuring Theta13 via Muon Neutrino to Electron Neutrino Oscillations in the MINOS Experiment. s.1. : University of Cambridge, 2012.

49. - . Ranking and Cutting Systematic Errors for the 4th Nue Analysis. s.1. : MINOS docdb-8904, 2012.

50. MINOS Calibration Group. 2009 Position Paper on Calibration of Runs I-II-III. s.1. : MINOS docdb-6717, 2009.

51. J. Boehm and M. Sanchez. Evaluation of systematic uncertainties on the measurement of Nue CC Appearance. s.1. : MINOS docdb-5387, 2008.

52. Pseudoscalar form factors in tau-neutrino nucleon scattering. Hagiwara et al. s.1. : Phys. Lett. B, 591:113-118, 2004.

53. Charged-particle multiplicity distributions in $v n$ and $v p$ charged-current interactions.

D. Zieminska et al. s.1. : Phys. Rev. D, 27:47-57, 1983.

54. Multiplicity distributions in nu-bar/sub. mu. /p interactions. M. Derrick et al. s.1. :

Phys. Rev. D, 25:3, 1982.

55. Schreckenberger, A.P. MRE Summary. s.1. : MINOS docdb-7963, 2011.

56. Sher, S.M. MRE Efficiencies from Event Reweighting. s.1. : MINOS docdb-9014, 2012.

57. An independent measurement of the total active B-8 solar neutrino flux using an array of He-3 proportional counters at the Sudbury Neutrino Observatory. B. Aharmim et al. s.1. : Phys. Rev. Lett. 101:111301, 2008.

58. MINOS Collaboration. MINOS Scientific Results. [Online] 2012. http://wwwnumi.fnal.gov/PublicInfo/forscientists.html.

59. Electron neutrino and antineutrino appearance in the complete MINOS data set. $\mathbf{P}$.

Adamson et al. s.1. : MINOS docdb-9315, 2012.

60. A unified approach to the classical statistical analysis of small signals. G.J. Feldman and R.D. Cousins. s.1. : Phys. Rev. D, 57:3873-3889, 1998.

61. TMinuit Package. [Online] http://root.cern.ch/root/html/TMinuit.html.

62. Schreckenberger, A.P. FHC Glass Box Opening. s.1. : MINOS docdb-9119, 2012.

63. S. Cavanaugh and L. Whitehead. Nue Far Detector Sidebands - Predictions and Results. s.1. : MINOS docdb-6705, 2010.

64. Cavanaugh, S. Far Data MRCC Update. s.1. : MINOS docdb-6596, 2009.

65. New Results from MINOS. Nichol, R. s.1. : Nucl. Phys. B Proceedings Supplement 00:1-7, 2012.

66. Indication of electron neutrino appearance from an accelerator-produced off-axis neutrino beam. K. Abe et al. (T2K). s.1. : Phys. Rev. Lett., 107:041801, 2011.

67. Proposal to build a 30 kiloton off-axis detector to study $v \mu \rightarrow$ ve oscillations in the NuMI beamline. D.S. Ayers et al (NOvA). s.1. : hep-ex/0503053, 2004.

68. Patterson, R.B. Summary of Theta13 Measurements. 2012.

69. Backhouse, C. Status of NOvA. [Online] 2012. http://nova-

docdb.fnal.gov/0079/007984/002/nova.pdf.

70. LoI - KATRIN: A next generation tritium beta decay experiment with sub-eV

sensitivity for the electron neutrino mass. A. Osipowicz et al. s.1. : hep-ex/0109033, 2001. 
71. COBRA: Double betal decay searches using CdTe detectors. Zuber, K. s.l. : Phys. Rev. Lett. B, 519:1-7, 2001.

72. Search for Neutrinoless Double-Beta Decay in 136Xe with EXO-200. M. Auger et al. s.1. : Phys. Rev. Lett. 109:032505, 2012.

73. The GERmanium Detector Array (GERDA) for the search of neutrinoless double beta decays of Ge-76 at LNGS. S. Schonert et al. s.1. : Nucl. Phys. Proc. Suppl. 145:242-245, 2005.

74. The Majorana Experiment. E. Aguayo et al. s.1. : arXiv:1109.4790, 2011.

75. The NEXT experiment: A high pressure xenon gas TPC for neutrinoless double beta decay searches. D. Lorca et al. s.1. : arXiv:1210.4020, 2012.

76. Neutrino detection in the ArgoNeuT LAr TPC. Palamara, O. s.1. : arXiv:1110.3070, 2011.

77. Precise 3D track reconstruction algorithm for the ICARUS T600 liquid argon time projection chamber detector. M. Antonello et al. s.1. : arXiv:1210.5089, 2012.

78. The Status of The MicroBooNE Experiment. Jones, B.J.P. s.1. : arXiv:1110.1678, 2011.

79. J. Strait et al. [Online] 2010. http://lbne2-

docdb.fnal.gov:8080/0030/003056/003/KeyAssumptions-PhysicsGoals_V1.0.pdf. 